\title{
Thermal form factors of the XXZ chain and the large-distance asymptotics of its temperature dependent correlation functions
}

\author{
Maxime Dugave^, Frank Göhmann \\ Fachbereich C - Physik, Bergische Universität Wuppertal, \\ 42097 Wuppertal, Germany \\ Karol K. Kozlowski周 \\ IMB, UMR 5584 du CNRS, Université de Bourgogne, France
}

\begin{abstract}
We derive expressions for the form factors of the quantum transfer matrix of the spin- $\frac{1}{2}$ XXZ chain which are suitable for taking the infinite Trotter number limit. These form factors determine the finitely many amplitudes in the leading asymptotics of the finite-temperature correlation functions of the model. We consider form-factor expansions of the longitudinal and transversal two-point functions. Remarkably, the formulae for the amplitudes are in both cases of the same form. We also explain how to adapt our formulae to the description of ground state correlation functions of the finite chain. The usefulness of our novel formulae is demonstrated by working out explicit results in the high- and low-temperature limits. We obtain, in particular, the large-distance asymptotics of the longitudinal twopoint functions for small temperatures by summing up the asymptotically most relevant terms in the form factor expansion of a generating function of the longitudinal correlation functions. As expected the leading term in the expansion of the corresponding two-point functions is in accordance with conformal field theory predictions. Here it is obtained for the first time by a direct calculation.
\end{abstract}

PACS: 05.30.-d, 75.10.Pq

\footnotetext{
*e-mail: dugave@uni-wuppertal.de

†e-mail: goehmann@uni-wuppertal.de

‡e-mail: karol.kozlowski@u-bourgogne.fr
} 


\section{Introduction}

For a long time form factors have been the main tool for studying the correlation functions of integrable massive quantum field theories through their Lehmann representation [20,47]. For these theories they can be determined as solutions of appropriate functional equations [48,49].

Form factors of integrable non-relativistic models can be studied by means of the vertex operator approach [18] and by means of the algebraic Bethe ansatz [23, 25,27, [28, 46]. In this work we apply the latter approach in order to derive expressions for the form factors of the quantum transfer matrix [51,52] that are suitable for taking the limit of an infinite Trotter number. Similar formulae hold for the form factors of the usual row-to-row transfer matrix for finite chains. In both cases the form factors will be described in terms of solutions of linear and non-linear integral equations, similar to those appearing in the description of the thermodynamics [29,30] and correlation functions [4, 14] of the model. We shall call such form factors thermal form factors.

At finite temperature the correlation functions of the XXZ chain decay exponentially. Their asymptotic behaviour is described by correlation lengths, determined by the ratio of one of the sub-dominant eigenvalues of the quantum transfer matrix and its dominant eigenvalue, and by amplitudes given as products of two corresponding thermal form factors. While the correlations lengths were studied thoroughly in the literature [29, 30, 33, 43] , so far rather little attention has been paid to the amplitudes.

Our expressions for the form factors involve, except for integrals over functions obtained as solutions of integral equations, also Fredholm determinants of the corresponding integral operators. We consider form factors arising in expansions of a generating function of the longitudinal correlation functions and of the transversal two-point correlation functions. The usefulness of our formulae is demonstrated in several limiting cases for high and low temperatures.

The analysis of the low-temperature limit is delicate and cumbersome. In this work we concentrate on the longitudinal correlation functions in the critical regime. The low-temperature analysis of the transversal correlation functions in the critical regime is similar but requires a separate calculation which we leave for future work. Quite generally, critical models at low temperature are characterized by the fact that infinitely many of their correlation lengths diverge [10,11]. We shall see that the corresponding amplitudes vanish algebraically as functions of the temperature with the same critical exponents that govern the spatial decay of the ground state correlation functions. The situation is very much like for the finite-size dependence of the 'critical amplitudes' occurring in the form factor expansion of the ground state correlation functions [23, 25]. A similar temperature dependence was also reported for the amplitudes in a series expansion of the density-density correlation functions of the Bose gas with deltafunction interactions [37,38] obtained from a multiple-integral representation of the generating function.

In fact, we shall closely follow the work [37] in our low-temperature analysis. This allows us to keep the presentation short, as many details can be proved along the lines of [37]. As in [37] we can also sum up the critical particle-hole contributions to the form factor expansion by means of a summation formula proved in [21,24, 40]. As a result we obtain the low-temperature large-distance asymptotics of the longitudinal correlation functions. The form of the exponential decay is in accordance with conformal field 
theory predictions [10,11]. The amplitude of the leading oscillating term so far could not be obtained by approximate field theoretical calculations. It is the same as the ground state's leading oscillatory amplitude which was obtained by a direct asymptotic analysis of multiple integrals in [22].

\section{Hamiltonian and correlation functions}

The XXZ-chain in a longitudinal magnetic field $h$ is defined by the Hamiltonian

$$
H=J \sum_{j=-L+1}^{L}\left(\sigma_{j-1}^{x} \sigma_{j}^{x}+\sigma_{j-1}^{y} \sigma_{j}^{y}+\Delta\left(\sigma_{j-1}^{z} \sigma_{j}^{z}-1\right)\right)-\frac{h}{2} \sum_{j=-L+1}^{L} \sigma_{j}^{z},
$$

acting on $\left(\mathbb{C}^{2}\right)^{\otimes 2 L}$. Here the $\sigma_{j}^{\alpha}, \alpha=x, y, z$, are the Pauli matrices and periodic boundary conditions, $\sigma_{-L}^{\alpha}=\sigma_{L}^{\alpha}$, are implied. $J$ and $\Delta$ are the exchange energy and the anisotropy parameter. We shall employ the standard parameterization $\Delta=\left(q+q^{-1}\right) / 2, q=\mathrm{e}^{\eta}$.

The Hamiltonian (1) is integrable with $R$-matrix

$$
\begin{aligned}
& R(\lambda, \mu)=\left(\begin{array}{cccc}
1 & 0 & 0 & 0 \\
0 & b(\lambda, \mu) & c(\lambda, \mu) & 0 \\
0 & c(\lambda, \mu) & b(\lambda, \mu) & 0 \\
0 & 0 & 0 & 1
\end{array}\right), \\
& b(\lambda, \mu)=\frac{\operatorname{sh}(\lambda-\mu)}{\operatorname{sh}(\lambda-\mu+\eta)}, \quad c(\lambda, \mu)=\frac{\operatorname{sh}(\eta)}{\operatorname{sh}(\lambda-\mu+\eta)} .
\end{aligned}
$$

For the exact calculation of thermal averages [14] at temperature $T$ we have to express the statistical operator of the grand-canonical ensemble

$$
\rho_{L}=\mathrm{e}^{-H / T}
$$

in terms of the $R$-matrix (2a). For this purpose we introduce an auxiliary vertex model with monodromy matrix

$$
T_{j}(\lambda)=q^{\mathrm{K} \sigma_{j}^{z}} R_{j \bar{N}}\left(\lambda, \frac{\beta}{N}\right) R_{\overline{N-1} j}^{t_{1}}\left(-\frac{\beta}{N}, \lambda\right) \ldots R_{j \overline{2}}\left(\lambda, \frac{\beta}{N}\right) R_{\overline{1} j}^{t_{1}}\left(-\frac{\beta}{N}, \lambda\right) .
$$

It acts non-trivially in the tensor product of an 'auxiliary space' $j=-L+1, \ldots, L$ and $N \in 2 \mathbb{N}$ 'quantum spaces' $\overline{1}, \ldots, \bar{N}$ which are all isomorphic to $\mathbb{C}^{2}$. The superscript $t_{1}$ denotes the transposition with respect to the first of the two spaces $R(\lambda, \mu)$ is acting on. The parameters $\beta$ and $\kappa$ are related to the temperature $T$ and to the magnetic field $h$,

$$
\beta=\frac{2 J \operatorname{sh}(\eta)}{T}, \quad \kappa=\frac{h}{2 \eta T} .
$$

$T_{j}(\lambda)$ is a $2 \times 2$ matrix in auxiliary space $j$. Its matrix elements satisfy the Yang-Baxter algebra relations

$$
R_{j k}(\lambda, \mu) T_{j}(\lambda) T_{k}(\mu)=T_{k}(\mu) T_{j}(\lambda) R_{j k}(\lambda, \mu)
$$

by construction. 
Using the monodromy matrix (4) we can express the statistical operator (3) as a limit,

$$
\rho_{L}=\lim _{N \rightarrow \infty} \operatorname{Tr}_{\overline{1} . \ldots \bar{N}}\left\{T_{-L+1}(0) \ldots T_{L}(0)\right\}
$$

We call this limit the Trotter limit and the number $N$ the Trotter number. For finite Trotter number the operator

$$
\rho_{N, L}=\operatorname{Tr}_{\overline{1} \ldots \bar{N}}\left\{T_{-L+1}(0) \ldots T_{L}(0)\right\}
$$

approximates the statistical operator. It can be used to define finite Trotter number approximants of correlation functions of $m$ local operators $\mathcal{O}^{(1)}, \ldots, \mathcal{O}^{(m)}$ acting on sites $1, \ldots, m$ of the infinite chain

$$
\begin{gathered}
\left\langle\mathcal{O}_{1}^{(1)} \ldots \mathcal{O}_{m}^{(m)}\right\rangle_{N}=\lim _{L \rightarrow \infty} \frac{\operatorname{Tr}_{-L+1 \ldots L}\left\{\rho_{N, L} \mathcal{O}_{1}^{(1)} \ldots \mathcal{O}_{m}^{(m)}\right\}}{\operatorname{Tr}_{-L+1 \ldots L}\left\{\rho_{N, L}\right\}} \\
=\lim _{L \rightarrow \infty} \frac{\operatorname{Tr}_{\overline{1} \ldots \bar{N}}\left\{\operatorname{Tr}^{L}\{T(0)\} \operatorname{Tr}\left\{\mathcal{O}^{(1)} T(0)\right\} \ldots \operatorname{Tr}\left\{\mathcal{O}^{(m)} T(0)\right\} \operatorname{Tr}^{L-m}\{T(0)\}\right\}}{\operatorname{Tr}_{\overline{1} \ldots \bar{N}}\left\{\operatorname{Tr}^{2 L}\{T(0)\}\right\}}
\end{gathered}
$$

Here the expression

$$
Z_{N, L}=\operatorname{Tr}_{\overline{1} \ldots \bar{N}}\left\{\operatorname{Tr}^{2 L}\{T(0)\}\right\}
$$

in the denominator is the finite Trotter number approximant to the partition function $Z_{L}=\operatorname{Tr}_{-L+1 \ldots L}\{\exp (-H / T)\}$ of the XXZ chain of length $2 L$. The operator occurring on the right hand side of equation $(10)$,

$$
t(\lambda)=\operatorname{Tr}\{T(\lambda)\}
$$

is called the quantum transfer matrix [51]. It has the peculiar property that the spectrum of $t(0)$ contains a unique real eigenvalue $\Lambda_{0}(0)$, whose modulus is strictly larger than those of all other eigenvalues, even if the Hamiltonian (1) is in the critical regime $|\Delta|<1$. This implies that $t(0)^{L}$ in 9$\}$ acts as a projector onto the corresponding eigenstate $\left|\Psi_{0}\right\rangle$, when $L$ becomes large. It follows that

$$
\left\langle\mathcal{O}_{1}^{(1)} \ldots \mathcal{O}_{m}^{(m)}\right\rangle_{N}=\frac{\left\langle\Psi_{0}\left|\operatorname{Tr}\left\{\mathcal{O}^{(1)} T(0)\right\} \ldots \operatorname{Tr}\left\{\mathcal{O}^{(m)} T(0)\right\}\right| \Psi_{0}\right\rangle}{\left\langle\Psi_{0} \mid \Psi_{0}\right\rangle \Lambda_{0}^{m}(0)} .
$$

We shall call $\left|\Psi_{0}\right\rangle$ the dominant eigenstate and $\Lambda_{0}(\lambda)$ the dominant eigenvalue. We see that the dominant eigenstate determines all thermal correlation functions of the system in the thermodynamic limit, or, to phrase it differently, that it determines the state of thermal equilibrium completely. The physical correlation functions follow from (12) in the Trotter limit $N \rightarrow \infty$. For more details see [14].

\section{Bethe ansatz and auxiliary functions}

Eigenvectors and eigenvalues of the quantum transfer matrix can be constructed by means of the algebraic Bethe ansatz. This follows since the monodromy matrix elements, for which we use the standard notation

$$
T(\lambda)=\left(\begin{array}{ll}
A(\lambda) & B(\lambda) \\
C(\lambda) & D(\lambda)
\end{array}\right)
$$


satisfy the Yang-Baxter algebra relation $(6)$ and since a pseudo vacuum $|0\rangle=\left[\left(\begin{array}{l}0 \\ 1\end{array}\right) \otimes\right.$ $\left.\left(\begin{array}{l}1 \\ 0\end{array}\right)\right]^{\otimes N / 2}$ exists on which the monodromy matrix elements act as

$$
\begin{aligned}
& C(\lambda)|0\rangle=0, \\
& A(\lambda)|0\rangle=q^{\kappa} b^{\frac{N}{2}}\left(-\frac{\beta}{N}, \lambda\right)|0\rangle=a(\lambda)|0\rangle, \\
& D(\lambda)|0\rangle=q^{-\kappa} b^{\frac{N}{2}}\left(\lambda, \frac{\beta}{N}\right)|0\rangle=d(\lambda)|0\rangle .
\end{aligned}
$$

Then (see e.g. [36])

$$
\left|\Psi_{n}\right\rangle=B\left(\lambda_{M}\right) \ldots B\left(\lambda_{1}\right)|0\rangle
$$

is an eigenvector of the quantum transfer matrix $t(\lambda)$ if the Bethe roots $\lambda_{j}, j=1, \ldots, M$, satisfy the system

$$
\frac{a\left(\lambda_{j}\right)}{d\left(\lambda_{j}\right)}=\prod_{\substack{k=1 \\ k \neq j}}^{M} \frac{\operatorname{sh}\left(\lambda_{j}-\lambda_{k}+\eta\right)}{\operatorname{sh}\left(\lambda_{j}-\lambda_{k}-\eta\right)}
$$

of Bethe ansatz equations. We note that (16) has many solutions, for $M=0, \ldots, N$, and that every eigenstate $\left|\Psi_{n}\right\rangle$ is parametrized by its own characteristic set of Bethe roots, which therefore depends on $n$. We could exhibit this dependence writing $\lambda_{j}^{(n)}$ and $M_{n}$ instead of $\lambda_{j}$ and $M$, but, for the sake of brevity, we follow the tradition and refrain from such explicit notation.

The eigenvalue corresponding to $\left|\Psi_{n}\right\rangle$ is

$$
\Lambda_{n}(\lambda)=a(\lambda) \prod_{j=1}^{M} \frac{\operatorname{sh}\left(\lambda-\lambda_{j}-\eta\right)}{\operatorname{sh}\left(\lambda-\lambda_{j}\right)}+d(\lambda) \prod_{j=1}^{M} \frac{\operatorname{sh}\left(\lambda-\lambda_{j}+\eta\right)}{\operatorname{sh}\left(\lambda-\lambda_{j}\right)}
$$

and a left eigenvector with the same eigenvalue can be written as

$$
\left\langle\Psi_{n}\right|=\langle 0| C\left(\lambda_{1}\right) \ldots C\left(\lambda_{M}\right),
$$

where $\langle 0|=| 0\rangle^{t}$ is the left pseudo vacuum satisfying $\langle 0| B(\lambda)=0$.

We have used $n=0,1, \ldots, 2^{N}-1$ to enumerate the eigenstates. This means we assume implicitly that, for generic $q$ and non-zero $\kappa$, the sets of right and left eigenvectors defined by $(15)$ and 18$)$ form bases of $\left(\mathbb{C}^{2}\right)^{\otimes N}$ and of its dual. We would like to emphasize that this point is important for the interpretation of our results below, but not so much for the results themselves. The quantum transfer matrix of the XXZ chain can be seen as a special inhomogeneous case of the usual transfer matrix of the model (see e.g. [4]). For a discussion of the issue of completeness of the Bethe states of the latter, as far as it is relevant in the present context, the reader is referred to [27]. In the following we shall assume that the eigenvalues are ordered by size in such a way that $\Lambda_{0}(0)>\left|\Lambda_{1}(0)\right| \geq\left|\Lambda_{2}(0)\right| \geq \ldots$

Along with the quantum transfer matrix $t(\lambda)$ we consider a 'twisted version' $t(\lambda \mid \alpha)$ obtained from $t(\lambda)$ by shifting $\kappa \rightarrow \kappa+\alpha$. Then $a(\lambda) \rightarrow q^{\alpha} a(\lambda), d(\lambda) \rightarrow q^{-\alpha} d(\lambda)$ and the Bethe roots are altered as well. For the corresponding eigenvalues and eigenvectors we use the notation $\Lambda_{n}(\lambda \mid \alpha)$ and $\left|\Psi_{n}^{\alpha}\right\rangle$.

Our goal is to write the form factors of the quantum transfer matrix in a form that admits the Trotter limit. For this purpose we introduce certain auxiliary functions generalizing some of those functions that proved to be useful in the description of the 
finite-temperature correlation functions of the model [4, 14]. For every regular solution $\$$ of the Bethe equations $(16)$ we define an auxiliary function

$$
\mathfrak{a}_{n}(\lambda)=\frac{d(\lambda)}{a(\lambda)} \prod_{j=1}^{M} \frac{\operatorname{sh}\left(\lambda-\lambda_{j}+\eta\right)}{\operatorname{sh}\left(\lambda-\lambda_{j}-\eta\right)} .
$$

In the twisted case we write $\mathfrak{a}_{n}(\lambda \mid \alpha)$. We also define

$$
\rho_{n}(\lambda \mid \alpha)=\frac{\Lambda_{n}(\lambda \mid \alpha)}{\Lambda_{0}(\lambda)}
$$

In the Trotter limit the functions $\mathfrak{a}_{n}(\lambda)$ are uniquely determined by the integral equation

$$
\ln \left(\mathfrak{a}_{n}(\lambda)\right)=-(2 \kappa+N-2 M) \eta-\beta \mathrm{e}(\lambda)-\int_{\mathfrak{C}_{n}} \frac{\mathrm{d} \mu}{2 \pi \mathrm{i}} K(\lambda-\mu) \ln \left(1+\mathfrak{a}_{n}(\mu)\right) .
$$

Here we have introduced the kernel

$$
K(\lambda)=K_{0}(\lambda), \quad K_{\alpha}(\lambda)=q^{-\alpha} \operatorname{cth}(\lambda-\eta)-q^{\alpha} \operatorname{cth}(\lambda+\eta)
$$

which is the derivative of the bare two-particle scattering phase, and the bare energy

$$
\mathrm{e}(\lambda)=\operatorname{cth}(\lambda)-\operatorname{cth}(\lambda+\eta)
$$

In equation (21) the information about the specific excitation is hidden in the choice of the integration contours $\mathcal{C}_{n}$. These are deformations of the contour $\mathcal{C}_{0}$ pertaining to the dominant state $\left|\Psi_{0}\right\rangle$. We have to distinguish between the massive and the massless case $\Delta>1$ and $|\Delta|<1$, respectively. In the massive case $\eta$ is real and the contour $\mathrm{C}_{0}$ is a finite rectangle oriented parallel to the real and imaginary axis and cutting the axes at $\pm \eta / 2$ and $\pm i \pi / 2$ (for a picture see [14]). In the massless case $\eta=-i \gamma$, $\gamma \in(0, \pi)$, and the contour $\mathcal{C}_{0}$ consists of two straight lines parallel to the real axis at $\pm \mathrm{i} \min \{\gamma / 2, \pi / 2-\gamma / 2\}$, encircling the real axis in positive direction.

All Bethe roots of the dominant state are located inside $\mathcal{C}_{0}$. All zeros of $\Lambda_{0}(\lambda \mid \alpha)$, which are zeros of $1+\mathfrak{a}_{0}(\lambda \mid \alpha)$ as well, are located outside $\mathcal{C}_{0}$. These zeros are called holes. If $\lambda$ is on the contour we infinitesimally deform it in such a way that the point at the opposite side of the contour $(\lambda+\eta$ or $\lambda-\eta)$ is outside. Excitations are characterized by a finite number of holes $\lambda_{j}^{h}$ inside $\mathcal{C}_{0}$ and a finite number of Bethe roots $\lambda_{k}^{p}$ outside. The contours $\mathcal{C}_{n}$ are obtained by deforming $\mathcal{C}_{0}$ in such a way that the $\lambda_{j}^{h}$ are excluded from $\mathcal{C}_{n}$ but the $\lambda_{k}^{p}$ are included (see figure 1 . For our convenience we shall also assume that all Bethe roots of the dominant state are located inside $\mathcal{C}_{n}$ while the corresponding holes remain outside 1

The contours $\mathcal{C}_{n}$ can be straightened. Then modified driving terms depending explicitly on the particle and hole parameters $\lambda_{k}^{p}$ and $\lambda_{j}^{h}$ appear, which can be determined by a set of subsidiary conditions of the form $\mathfrak{a}_{n}\left(\lambda_{k}^{p}\right)=-1, \mathfrak{a}_{n}\left(\lambda_{j}^{h}\right)=-1$ (see [30]). Such type of formulation is useful for numerical calculations and for the low-temperature

\footnotetext{
${ }^{\S}$ We call a solution of the Bethe equations regular if it corresponds to an eigenvector of the form 15 .

IThis implies that we exclude the free Fermion case $\gamma=\pi / 2$ from our considerations.
} 


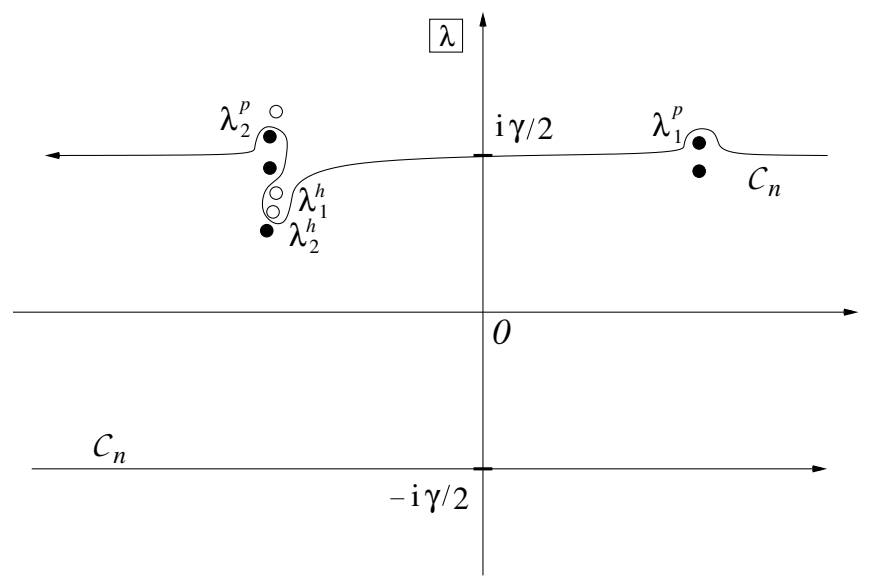

Figure 1: Sketch of a contour $\mathcal{C}_{n}$ for $0<\Delta<1$. Hole parameters $\lambda_{j}^{h}$ inside the 'physical strip' between $-\mathrm{i} \gamma / 2$ and $\mathrm{i} \gamma / 2$ are excluded from the contour, and particle parameters (Bethe roots) $\lambda_{k}^{p}$ outside the physical strip are included into the contour.

analysis of section 8 . For the derivation of form-factor formulae, however, the deformed contour formulation seems to be more convenient.

In order to keep our exposition simple we shall restrict the range of $\Delta$ in the following to $0<\Delta<1$. The range $-1<\Delta<0$ can be accessed by a canonical transformation [56]. Also note that our results, except for those in section 8 on the low-temperature limit, are valid in the massive case as well

The functions $\rho_{n}(\lambda \mid \alpha)$ can be represented as integrals over the auxiliary functions,

$$
\rho_{n}(\lambda \mid \alpha)=q^{\alpha+\frac{N}{2}-M} \exp \left\{\int_{\mathfrak{C}_{n}} \frac{\mathrm{d} \mu}{2 \pi \mathrm{i}} \mathrm{e}(\mu-\lambda) \ln \left(\frac{1+\mathfrak{a}_{n}(\mu \mid \alpha)}{1+\mathfrak{a}_{0}(\mu)}\right)\right\}
$$

where $\lambda$ is located inside the contour $\mathcal{C}_{n}$. The number $N / 2-M$ is the eigenvalue of the conserved $z$-component of the pseudo spin, $\eta^{z}=\frac{1}{2} \sum_{j=1}^{N}(-1)^{j} \sigma_{j}^{z}$. In our discussion of the form factors below we have to distinguish two cases: (i) $N / 2-M=0$, which is relevant for the longitudinal correlation functions, and (ii) $N / 2-M=1$, which applies to the transversal correlation functions. In both cases the right hand side of (24) is well defined and non-trivial in the Trotter limit.

\section{Form factor expansion of correlation functions}

We shall consider form factor expansions of the longitudinal and transversal two-point functions $\left\langle\sigma_{1}^{z} \sigma_{m+1}^{z}\right\rangle$ and $\left\langle\sigma_{1}^{-} \sigma_{m+1}^{+}\right\rangle$. For the longitudinal two-point functions we start with a generating function [17] which is closely related to the twisted transfer matrix. Setting

$$
S(m)=\frac{1}{2} \sum_{j=1}^{m} \sigma_{j}^{z}
$$

\footnotetext{
${ }^{\|}$Possibly certain phase factors stemming from integration boundaries have to be adapted.
} 
the generating function is defined as $\left\langle q^{2 \alpha S(m)}\right\rangle$, where $\alpha$ is a complex parameter. In fact,

$$
\left\langle\sigma_{1}^{z} \sigma_{m+1}^{z}\right\rangle=\left.\frac{1}{2} D_{m}^{2} \partial_{\eta \alpha}^{2}\left\langle q^{2 \alpha S(m+1)}\right\rangle\right|_{\alpha=0}
$$

where $D_{m}$ is the difference operator defined by $D_{m} f(m)=f(m)-f(m-1)$. Note that the generating function provides more information. The one-point functions are given by

$$
\left\langle\sigma_{1}^{z}\right\rangle=\left.D_{m} \partial_{\eta \alpha}\left\langle q^{2 \alpha S(m+1)}\right\rangle\right|_{\alpha=0},
$$

and the emptiness formation probability can be obtained as the limit

$$
\left\langle e_{1+}^{+} \ldots e_{m+}^{+}\right\rangle=\lim _{\operatorname{Re} \eta \alpha \rightarrow+\infty} q^{-m \alpha}\left\langle q^{2 \alpha S(m)}\right\rangle .
$$

Here $e_{j_{+}}^{+}$is a projector onto the local spin-up state.

It follows from (12) that

$$
\left\langle q^{2 \alpha S(m)}\right\rangle_{N}=\frac{\left\langle\Psi_{0}\left|t^{m}(0 \mid \alpha)\right| \Psi_{0}\right\rangle}{\left\langle\Psi_{0} \mid \Psi_{0}\right\rangle \Lambda_{0}^{m}(0)}
$$

is the finite Trotter number approximant to the generating function. Expanding $t^{m}(0 \mid \alpha)\left|\Psi_{0}\right\rangle$ in the basis of Bethe states $\left\{\left|\Psi_{n}^{\alpha}\right\rangle\right\}$ we obtain the form factor expansion

$$
\left\langle q^{2 \alpha S(m)}\right\rangle_{N}=\sum_{n=0}^{N_{M}-1} A_{n}(\alpha) \rho_{n}^{m}(0 \mid \alpha), \quad A_{n}(\alpha)=\frac{\left\langle\Psi_{0} \mid \Psi_{n}^{\alpha}\right\rangle\left\langle\Psi_{n}^{\alpha} \mid \Psi_{0}\right\rangle}{\left\langle\Psi_{0} \mid \Psi_{0}\right\rangle\left\langle\Psi_{n}^{\alpha} \mid \Psi_{n}^{\alpha}\right\rangle}
$$

Here we have adapted the numbering of the states. We count only those $N_{M}=\left(\begin{array}{l}N \\ M\end{array}\right)$, $M=N / 2$, states which have non-zero overlap with the dominant state.

Inserting now (30) into 26) we obtain the form factor expansion of the connected longitudinal correlation functions,

$$
\left\langle\sigma_{1}^{z} \sigma_{m+1}^{z}\right\rangle_{N}-\left\langle\sigma_{1}^{z}\right\rangle_{N}\left\langle\sigma_{m+1}^{z}\right\rangle_{N}=\sum_{n=1}^{N_{M}-1} A_{n}^{z z}\left(\rho_{n}^{\frac{1}{2}}-\rho_{n}^{-\frac{1}{2}}\right)^{2} \rho_{n}^{m} .
$$

In this expression we introduced the abbreviations

$$
\rho_{n}=\rho_{n}(0 \mid 0), \quad A_{n}^{z z}=\left.\frac{1}{2} \partial_{\eta \alpha}^{2} A_{n}(\alpha)\right|_{\alpha=0}=\frac{\left\langle\Psi_{0} \mid \Psi_{n}^{\prime}\right\rangle\left\langle\Psi_{n}^{\prime} \mid \Psi_{0}\right\rangle}{\left\langle\Psi_{0} \mid \Psi_{0}\right\rangle\left\langle\Psi_{n} \mid \Psi_{n}\right\rangle}
$$

By $\left|\Psi_{n}^{\prime}\right\rangle$ and $\left\langle\Psi_{n}^{\prime}\right|$ we mean the derivatives of the corresponding $\alpha$-dependent vectors with respect to $\eta \alpha$ at $\alpha=0$.

Note that $1>\left|\rho_{1}\right| \geq\left|\rho_{2}\right| \geq \ldots$ by construction. This means that (31) is the finitetemperature asymptotic expansion of the longitudinal correlation functions. The leading asymptotics is determined by the first few terms. The correlation length of the longitudinal correlation functions is

$$
\xi_{1}=-\frac{1}{\ln \left|\rho_{1}\right|}
$$

This correlation length was studied in [13, 30, 33, 43, 53]. So far the amplitudes in (31) were only studied numerically [13] for finite Trotter numbers. Below in section 5]we shall calculate them analytically in the Trotter limit. 
Using once more (12) we obtain a finite-temperature asymptotic expansion for the transversal correlation functions,

$$
\left\langle\sigma_{1}^{-} \sigma_{m+1}^{+}\right\rangle_{N}=\sum_{n=1}^{N_{M}} A_{n}^{-+} \rho_{n}^{m},
$$

where

$$
A_{n}^{-+}=\frac{\left\langle\Psi_{0}|B(0)| \Psi_{n}\right\rangle}{\Lambda_{n}(0)\left\langle\Psi_{0} \mid \Psi_{0}\right\rangle} \frac{\left\langle\Psi_{n}|C(0)| \Psi_{0}\right\rangle}{\Lambda_{0}(0)\left\langle\Psi_{n} \mid \Psi_{n}\right\rangle}
$$

Again the sum is restricted to the non-vanishing amplitudes (for which necessarily $M=N / 2-1$ ) and the numbering is adapted (in this case we start with ' 1 ', since we have reserved the label ' 0 ' for the dominant eigenstate). Accordingly the $\rho_{n}$ in the above formula (34) belong to excitations with $M=N / 2-1$ Bethe roots and are different from the $\rho_{n}$ in equation (31) belonging to excitations with $M=N / 2$ Bethe roots. As before the correlation length is defined by (33) with the appropriate $\rho_{1}$ inserted. The amplitudes in the Trotter limit were heretofore unknown and will be calculated in section 6 .

\section{Amplitudes in the asymptotic expansion of the longitudi- nal correlation functions}

The basic equation underlying the algebraic Bethe ansatz approach to the correlation functions of the XXZ chain is the following scalar product formula due to Nikita Slavnov [46]:

$$
\begin{array}{r}
\left\langle 0\left|C\left(\mu_{1}\right) \ldots C\left(\mu_{M}\right) B\left(\lambda_{M}\right) \ldots B\left(\lambda_{1}\right)\right| 0\right\rangle=\left\langle 0\left|C\left(\lambda_{1}\right) \ldots C\left(\lambda_{M}\right) B\left(\mu_{M}\right) \ldots B\left(\mu_{1}\right)\right| 0\right\rangle \\
=\left[\prod_{j=1}^{M} a\left(\mu_{j}\right) d\left(\lambda_{j}\right) \prod_{k=1}^{M} \frac{1}{b\left(\lambda_{j}, \mu_{k}\right)}\right] \frac{\operatorname{det}_{M}\left(\mathrm{e}\left(\lambda_{j}-\mu_{k}\right)-\mathrm{e}\left(\mu_{k}-\lambda_{j}\right) \mathfrak{a}_{n}\left(\mu_{k}\right)\right)}{\operatorname{det}_{M}\left(\frac{1}{\operatorname{sh}\left(\lambda_{j}-\mu_{k}\right)}\right)} .
\end{array}
$$

Here the $\lambda_{j}, j=1, \ldots, M$, are a solution of the Bethe equations 16 and $\mathfrak{a}_{n}$ is the associated auxiliary function. The $\mu_{j}$ in this formula are free. They may or may not be a solution of the Bethe equations. In particular, we can take the limit $\mu_{j} \rightarrow \lambda_{j}$ in 36 and obtain the 'norm formula' for Bethe states [35],

$$
\left\langle\Psi_{n} \mid \Psi_{n}\right\rangle=\left[\prod_{j=1}^{M} d\left(\lambda_{j}\right) \Lambda_{n}\left(\lambda_{j}\right)\right] \operatorname{det}_{M}\left\{\delta_{k}^{j}-\frac{K\left(\lambda_{j}-\lambda_{k}\right)}{\mathfrak{a}_{n}^{\prime}\left(\lambda_{j}\right)}\right\} .
$$

Using (36) and 37) in (32) or 35) we obtain expressions for the amplitudes in terms of Bethe roots (compare [28]). These are not directly suitable for taking the Trotter limit. Still, after a sequence of elementary manipulations (see appendix A) we obtain the following result,

$$
\begin{aligned}
A_{n}(\alpha)=\left[\prod_{j=1}^{M} \frac{\rho_{n}\left(\lambda_{j} \mid \alpha\right)}{\rho_{n}\left(\mu_{j} \mid \alpha\right)}\right] \frac{\operatorname{det}_{M}\left\{\delta_{k}^{j}-\frac{\rho_{n}\left(\mu_{j} \mid \alpha\right)}{\mathfrak{a}_{n}^{\prime}\left(\mu_{j} \mid \alpha\right)} \mathcal{K}_{-\alpha}\left(\mu_{j}-\mu_{k}\right)\right\}}{\operatorname{det}_{M}\left\{\delta_{k}^{j}-\frac{1}{\mathfrak{a}_{n}^{\prime}\left(\mu_{j} \mid \alpha\right)} \mathcal{K}\left(\mu_{j}-\mu_{k}\right)\right\}} \\
\times \frac{\operatorname{det}_{M}\left\{\delta_{k}^{j}-\frac{\rho_{n}^{-1}\left(\lambda_{j} \mid \alpha\right)}{\mathfrak{a}_{0}^{\prime}\left(\lambda_{j}\right)} \mathcal{K}_{\alpha}\left(\lambda_{j}-\lambda_{k}\right)\right\}}{\operatorname{det}_{M}\left\{\delta_{k}^{j}-\frac{1}{\mathfrak{a}_{0}^{\prime}\left(\lambda_{j}\right)} \mathcal{K}\left(\lambda_{j}-\lambda_{k}\right)\right\}} .
\end{aligned}
$$


In this equation $M=N / 2$, the $\lambda_{j}, j=1, \ldots, M$, are the Bethe roots of the dominant state, while the $\mu_{j}, j=1, \ldots, M$, are the Bethe roots of an excited state of the twisted transfer matrix $t(\lambda \mid \alpha)$. The kernel functions in the determinants are defined as

$$
\mathcal{K}(\lambda)=\mathcal{K}_{0}(\lambda), \quad \mathcal{K}_{\alpha}(\lambda)=\frac{\mathrm{e}^{(\alpha-1)(\lambda-\eta)}}{\operatorname{sh}(\lambda-\eta)}-\frac{\mathrm{e}^{(\alpha-1)(\lambda+\eta)}}{\operatorname{sh}(\lambda+\eta)} .
$$

The right hand side of (38) is suitable for taking the Trotter limit. For the product this is obvious from the representation

$$
\prod_{j=1}^{M} \frac{\rho_{n}\left(\lambda_{j} \mid \alpha\right)}{\rho_{n}\left(\mu_{j} \mid \alpha\right)}=\exp \left\{\int_{\mathfrak{C}_{n}} \frac{\mathrm{d} \lambda}{2 \pi \mathrm{i}} \frac{\rho_{n}^{\prime}(\lambda \mid \alpha)}{\rho_{n}(\lambda \mid \alpha)} \ln \left(\frac{1+\mathfrak{a}_{n}(\lambda \mid \alpha)}{1+\mathfrak{a}_{0}(\lambda)}\right)\right\}
$$

The determinants are all of the same structure. Expanding e.g. the first determinant in the numerator we obtain

$$
\begin{array}{r}
\operatorname{det}_{M}\left\{\delta_{k}^{j}-\frac{\rho_{n}\left(\mu_{j} \mid \alpha\right)}{\mathfrak{a}_{n}^{\prime}\left(\mu_{j} \mid \alpha\right)} \mathcal{K}_{-\alpha}\left(\mu_{j}-\mu_{k}\right)\right\}=1-\sum_{j=1}^{M} \frac{\rho_{n}\left(\mu_{j} \mid \alpha\right)}{\mathfrak{a}_{n}^{\prime}\left(\mu_{j} \mid \alpha\right)} \mathcal{K}_{-\alpha}(0) \\
+\sum_{1 \leq j<k \leq M} \frac{\rho_{n}\left(\mu_{j} \mid \alpha\right)}{\mathfrak{a}_{n}^{\prime}\left(\mu_{j} \mid \alpha\right)} \frac{\rho_{n}\left(\mu_{k} \mid \alpha\right)}{\mathfrak{a}_{n}^{\prime}\left(\mu_{k} \mid \alpha\right)} \operatorname{det}\left|\begin{array}{cc}
\mathcal{K}_{-\alpha}(0) & \mathcal{K}_{-\alpha}\left(\mu_{j}-\mu_{k}\right) \\
\mathcal{K}_{-\alpha}\left(\mu_{k}-\mu_{j}\right) & \mathcal{K}_{-\alpha}(0)
\end{array}\right|-\ldots \\
=1+\sum_{k=1}^{M} \frac{(-1)^{k}}{k !}\left[\prod_{j=1}^{k} \int_{\mathcal{C}_{n}} \mathrm{~d} m_{+}^{\alpha}\left(v_{j}\right)\right] \operatorname{det}_{k}\left\{\mathcal{K}_{-\alpha}\left(v_{l}-v_{m}\right)\right\}
\end{array}
$$

where we have introduced the 'measure'

$$
\mathrm{d} m_{+}^{\alpha}(\lambda)=\frac{\mathrm{d} \lambda \rho_{n}(\lambda \mid \alpha)}{2 \pi \mathrm{i}\left(1+\mathfrak{a}_{n}(\lambda \mid \alpha)\right)}
$$

In the Trotter limit $N=2 M \rightarrow \infty$ the right hand side of (41) converges to the Fredholm determinant of the integral operator $\widehat{\mathcal{K}}_{-\alpha}$ defined by the kernel $\mathcal{K}_{-\alpha}$, the measure $\mathrm{d} m_{+}^{\alpha}$ and the contour $\mathrm{C}_{n}$,

$$
\begin{aligned}
& \lim _{N \rightarrow \infty} \operatorname{det}\left\{\delta_{k}^{j}-\frac{\rho_{n}\left(\mu_{j} \mid \alpha\right)}{\mathfrak{a}_{n}^{\prime}\left(\mu_{j} \mid \alpha\right)} \mathcal{K}_{-\alpha}\left(\mu_{j}-\mu_{k}\right)\right\} \\
& =1+\sum_{k=1}^{\infty} \frac{(-1)^{k}}{k !}\left[\prod_{j=1}^{k} \int_{\mathcal{C}_{n}} \mathrm{~d} m_{+}^{\alpha}\left(v_{j}\right)\right] \operatorname{det}_{k}\left\{\mathcal{K}_{-\alpha}\left(v_{l}-v_{m}\right)\right\}=\operatorname{det}_{\mathrm{d} m_{+}^{\alpha}, \mathcal{C}_{n}}\left\{1-\widehat{\mathcal{K}}_{-\alpha}\right\} .
\end{aligned}
$$

The other determinants in (38) can be treated in a similar way. Introducing the measures

$$
\begin{aligned}
\mathrm{d} m(\lambda) & =\frac{\mathrm{d} \lambda}{2 \pi \mathrm{i}\left(1+\mathfrak{a}_{0}(\lambda)\right)}, \\
\mathrm{d} m_{0}^{\alpha}(\lambda) & =\frac{\mathrm{d} \lambda}{2 \pi \mathrm{i}\left(1+\mathfrak{a}_{n}(\lambda \mid \alpha)\right)}, \\
\mathrm{d} m_{-}^{\alpha}(\lambda) & =\frac{\mathrm{d} \lambda \rho_{n}^{-1}(\lambda \mid \alpha)}{2 \pi \mathrm{i}\left(1+\mathfrak{a}_{0}(\lambda)\right)}
\end{aligned}
$$


we obtain

$$
\begin{aligned}
A_{n}(\alpha)=\exp \left\{\int_{\mathfrak{C}_{n}} \frac{\mathrm{d} \lambda}{2 \pi \mathrm{i}} \frac{\rho_{n}^{\prime}(\lambda \mid \alpha)}{\rho_{n}(\lambda \mid \alpha)} \ln \left(\frac{1+\mathfrak{a}_{n}(\lambda \mid \alpha)}{1+\mathfrak{a}_{0}(\lambda)}\right)\right\} \\
\times \frac{\operatorname{det}_{\mathrm{d} m_{+}^{\alpha}, \mathfrak{C}_{n}}\left\{1-\widehat{\mathcal{K}}_{-\alpha}\right\} \operatorname{det}_{\mathrm{d} m_{-}^{\alpha}, \mathfrak{e}_{n}}\left\{1-\widehat{\mathcal{K}}_{\alpha}\right\}}{\operatorname{det}_{\mathrm{d} m_{0}^{\alpha}, e_{n}}\{1-\widehat{\mathcal{K}}\} \operatorname{det}_{\mathrm{d} m, \mathfrak{e}_{n}}\{1-\widehat{\mathcal{K}}\}} .
\end{aligned}
$$

for the amplitudes in the Trotter limit. At least at first sight this looks rather appealing. The amplitudes are entirely described in terms of functions which appeared earlier in the description of the thermodynamic properties, the correlation lengths and the correlation functions of the model. These are the auxiliary functions $\mathfrak{a}_{n}$ (see [30]), the eigenvalue ratios $\rho_{n}$ (see [4,8]) and the deformed kernel $\mathcal{K}_{\alpha}$.

The latter is a close relative of the deformed kernel $K_{\alpha}$, equation (22), which first appeared in the context of the description of the correlation functions of the XXZ chain in [4.7]. It seems that the kernel $\mathcal{K}_{\alpha}$ is more suitable in the analysis of the scaling limit towards conformal field theory [2,8], while $K_{\alpha}$ seems to be more convenient for the high-temperature analysis. This is also reflected in the different symmetry properties of the two kernels,

$$
\mathcal{K}_{\alpha}(-\lambda)=\mathcal{K}_{2-\alpha}(\lambda), \quad K_{\alpha}(-\lambda)=K_{-\alpha}(\lambda) .
$$

Since the two kernels are related by

$$
\mathrm{e}^{-\alpha \lambda} \mathcal{K}_{\alpha}(\lambda)=K_{\alpha}(\lambda)-q^{-\alpha}+q^{\alpha},
$$

the integral operators in the determinants in 45 differ from the corresponding integral operators with kernel $K_{\alpha}$ only by a one-dimensional projector. Hence, using also the fact that $\mathcal{K}_{0}=K_{0}=K$, we can rewrite (45) as

$$
\begin{aligned}
A_{n}(\alpha)=\bar{\sigma}_{+}^{-} \sigma_{-}^{-} \exp \left\{\int_{\mathcal{C}_{n}} \frac{\mathrm{d} \lambda}{2 \pi \mathrm{i}} \frac{\rho_{n}^{\prime}(\lambda \mid \alpha)}{\rho_{n}(\lambda \mid \alpha)} \ln \left(\frac{1+\mathfrak{a}_{n}(\lambda \mid \alpha)}{1+\mathfrak{a}_{0}(\lambda)}\right)\right\} \\
\times \frac{\operatorname{det}_{\mathrm{d} m_{+}^{\alpha}, \mathfrak{C}_{n}}\left\{1-\widehat{K}_{-\alpha}\right\} \operatorname{det}_{\mathrm{d} m_{-}^{\alpha}, \mathcal{C}_{n}}\left\{1-\widehat{K}_{\alpha}\right\}}{\operatorname{det}_{\mathrm{d} m_{0}^{\alpha}, \mathfrak{e}_{n}}\{1-\widehat{K}\} \operatorname{det}_{\mathrm{d} m, \mathfrak{C}_{n}}\{1-\widehat{K}\}},
\end{aligned}
$$

where

$$
\bar{\sigma}_{+}^{-}=\lim _{\operatorname{Re} \lambda \rightarrow-\infty} \bar{\sigma}_{+}(\lambda), \quad \sigma_{-}^{-}=\lim _{\operatorname{Re} \lambda \rightarrow-\infty} \sigma_{-}(\lambda)
$$

and where the two functions $\bar{\sigma}_{+}(\lambda), \sigma_{-}(\lambda)$ are generalizations to excited states of the generalized dressed charge functions introduced in [5]. They are defined by the integral equations

$$
\begin{aligned}
& \bar{\sigma}_{+}(\lambda)=1+\int_{\mathfrak{C}_{n}} \mathrm{~d} m_{+}^{\alpha}(\mu) K_{\alpha}(\lambda-\mu) \bar{\sigma}_{+}(\mu), \\
& \sigma_{-}(\lambda)=1+\int_{\mathfrak{C}_{n}} \mathrm{~d} m_{-}^{\alpha}(\mu) \sigma_{-}(\mu) K_{\alpha}(\mu-\lambda) .
\end{aligned}
$$

Using the techniques developed in [5] it is not difficult to show that

$$
\bar{\sigma}_{+}^{-} \sigma_{-}^{-}=\operatorname{ch}^{-2}\left\{\int_{\mathcal{C}_{n}} \frac{\mathrm{d} \lambda}{2 \pi \mathrm{i}} \ln \left(\frac{1+\mathfrak{a}_{n}(\lambda \mid \alpha)}{1+\mathfrak{a}_{0}(\lambda)}\right)\right\} .
$$


When both sets of rapidities in (36) satisfy the Bethe equation we obtain two different formulae by using either of the two corresponding auxiliary functions on the right hand side of the equation. In order to derive (45) we used both possibilities in a symmetric fashion and ended up with a particularly symmetric result. Using two times the same formula and also 37 we obtain alternative expressions for $A_{n}(\alpha)$ containing the square of one of the two determinants that appear in the numerator on the right hand side of (45) and a different prefactor. Comparing these asymmetric formulae with 45 we obtain the following interesting relation between the Fredholm determinants in the numerator,

$$
\begin{aligned}
\frac{\operatorname{det}_{\mathrm{d} m_{-}^{\alpha}, \mathcal{C}_{n}}\left\{1-\widehat{\mathcal{K}}_{\alpha}\right\}}{\operatorname{det}_{\mathrm{d} m_{+}^{\alpha}, \mathcal{C}_{n}}\left\{1-\widehat{\mathcal{K}}_{-\alpha}\right\}}=\exp \left\{\frac{\beta \rho_{n}^{\prime}(0 \mid \alpha)}{\rho_{n}(0 \mid \alpha)}-\int_{\mathcal{C}_{n}} \frac{\mathrm{d} \lambda}{\pi \mathrm{i}} \ln \left(\frac{1+\mathfrak{a}_{n}(\lambda \mid \alpha)}{1+\mathfrak{a}_{0}(\lambda)}\right)\right. \\
\left.+\int_{\mathcal{C}_{n}} \frac{\mathrm{d} \lambda}{2 \pi \mathrm{i}}\left[\frac{\Lambda_{n}^{\prime}(\lambda \mid \alpha)}{\Lambda_{n}(\lambda \mid \alpha)} \ln \left(1+\mathfrak{a}_{0}(\lambda)\right)-\frac{\Lambda_{0}^{\prime}(\lambda)}{\Lambda_{0}(\lambda)} \ln \left(1+\mathfrak{a}_{n}(\lambda \mid \alpha)\right)\right]\right\} .
\end{aligned}
$$

Equations (45) and (48) are the main results of this section. They are valid for finite Trotter number as well as in the Trotter limit. For finite Trotter number the right hand sides of these equations are represented by finitely many integrals (see (41)). We will show below that the high- and the low-temperature limits of the amplitudes can be extracted from these formulae. Their suitability for numerical calculations will be explored in future research.

\section{Amplitudes in the asymptotic expansion of the transversal correlation functions}

In this section we consider a slight generalization of the amplitudes $A_{n}^{-+}$, equation 35, depending on a spectral parameter $\xi$ and on a twist parameter $\alpha$. We define

$$
F_{-}(\xi)=\frac{\left\langle\Psi_{0}|B(\xi)| \Psi_{n}^{\alpha}\right\rangle}{\Lambda_{n}(\xi \mid \alpha)\left\langle\Psi_{0} \mid \Psi_{0}\right\rangle}, \quad F_{+}(\xi)=\frac{\left\langle\Psi_{n}^{\alpha}|C(\xi)| \Psi_{0}\right\rangle}{\Lambda_{0}(\xi)\left\langle\Psi_{n}^{\alpha} \mid \Psi_{n}^{\alpha}\right\rangle}
$$

and

$$
A_{n}^{-+}(\xi)=F_{-}(\xi) F_{+}(\xi) .
$$

For $F_{+}(\xi)$ we obtain in appendix $\mathrm{B}$

$$
F_{+}(\xi)=\frac{G_{+}^{-}(\xi) \mathrm{e}^{-\xi} \prod_{j=1}^{M} d\left(\lambda_{j}\right) \mathrm{e}^{\lambda_{j}}}{\prod_{j=1}^{M-1} \rho_{n}\left(\mu_{j} \mid \alpha\right) q^{-\alpha} d\left(\mu_{j}\right) \mathrm{e}^{\mu_{j}}} \frac{\operatorname{det}_{M-1}\left\{\delta_{k}^{j}-\frac{\rho_{n}\left(\mu_{j} \mid \alpha\right)}{\mathfrak{a}_{n}^{\prime}\left(\mu_{j} \mid \alpha\right)} K_{1-\alpha}\left(\mu_{j}-\mu_{k}\right)\right\}}{\operatorname{det}_{M-1}\left\{\delta_{k}^{j}-\frac{1}{\mathfrak{a}_{n}^{\prime}\left(\mu_{j} \mid \alpha\right)} K\left(\mu_{j}-\mu_{k}\right)\right\}} .
$$

Here

$$
G_{+}^{-}(\xi)=\lim _{\operatorname{Re} \lambda \rightarrow-\infty} G_{+}(\lambda, \xi),
$$

and $G_{+}(\lambda, \xi)$ is the solution of the linear integral equation

$$
\begin{aligned}
G_{+}(\lambda, \xi)= & -\operatorname{cth}(\lambda-\xi) \\
& +q^{\alpha-1} \rho_{n}(\xi \mid \alpha) \operatorname{cth}(\lambda-\xi-\eta)+\int_{\mathcal{C}_{n}} \mathrm{~d} m_{+}^{\alpha}(\mu) K_{1-\alpha}(\lambda-\mu) G_{+}(\mu, \xi)
\end{aligned}
$$


The simplest way to obtain a formula for the amplitudes from 55 is to combine (55) with (36) and (37). Then

$$
\begin{aligned}
A_{n}^{-+}(\xi)= & \frac{F_{+}^{2}(\xi)\left\langle\Psi_{n}^{\alpha} \mid \Psi_{n}^{\alpha}\right\rangle}{\rho_{n}(\xi \mid \alpha)\left\langle\Psi_{0} \mid \Psi_{0}\right\rangle}= \\
& \frac{G_{+}^{-}(\xi)^{2} \mathrm{e}^{2\left(\sum_{j=1}^{M} \lambda_{j}-\sum_{j=1}^{M-1} \mu_{j}-\xi\right)} \prod_{j=1}^{M} d\left(\lambda_{j}\right)}{\rho_{n}(\xi \mid \alpha) \prod_{j=1}^{M-1} q^{-\alpha} d\left(\mu_{j}\right) \rho_{n}\left(\mu_{j} \mid \alpha\right)} \frac{\prod_{j=1}^{M-1} \Lambda_{0}\left(\mu_{j}\right)}{\prod_{j=1}^{M} \Lambda_{0}\left(\lambda_{j}\right)} \\
& \times \frac{\operatorname{det}_{M-1}^{2}\left\{\delta_{k}^{j}-\frac{\rho_{n}\left(\mu_{j} \mid \alpha\right)}{\mathfrak{a}_{n}^{\prime}\left(\mu_{j} \mid \alpha\right)} K_{1-\alpha}\left(\mu_{j}-\mu_{k}\right)\right\}}{\operatorname{det}_{M-1}\left\{\delta_{k}^{j}-\frac{1}{\mathfrak{a}_{n}^{\prime}\left(\mu_{j} \mid \alpha\right)} K\left(\mu_{j}-\mu_{k}\right)\right\} \operatorname{det}_{M}\left\{\delta_{k}^{j}-\frac{1}{\mathfrak{a}_{0}^{\prime}\left(\lambda_{j}\right)} K\left(\lambda_{j}-\lambda_{k}\right)\right\}} .
\end{aligned}
$$

As before the determinants turn into Fredholm determinants in the Trotter limit. Some more care is necessary when dealing with the prefactors. All in all we obtain

$$
\begin{aligned}
A_{n}^{-+}(\xi)=C(\xi) \exp \left\{\int_{\mathcal{C}_{n}} \frac{\mathrm{d} \lambda}{2 \pi \mathrm{i}} \frac{\rho_{n}^{\prime}(\lambda \mid \alpha)}{\rho_{n}(\lambda \mid \alpha)}\right. & \left.\ln \left(\frac{1+\mathfrak{a}_{n}(\lambda \mid \alpha)}{1+\mathfrak{a}_{0}(\lambda)}\right)\right\} \\
& \times \frac{\operatorname{det}_{\mathrm{d} m_{+}^{\alpha}, \mathcal{C}_{n}}^{2}\left\{1-\widehat{K}_{1-\alpha}\right\}}{\operatorname{det}_{\mathrm{d} m_{0}^{\alpha}, \mathfrak{C}_{n}}\{1-\widehat{K}\} \operatorname{det}_{\mathrm{d} m, \mathcal{C}_{n}}\{1-\widehat{K}\}},
\end{aligned}
$$

where

$$
\begin{aligned}
C(\xi)=\frac{G_{+}^{-}(\xi)^{2} \mathrm{e}^{-2 \xi}}{\rho_{n}(\xi \mid \alpha)} \frac{q^{\alpha+\kappa-1}+q^{-\alpha-\kappa+1}}{\left(q^{\kappa}+q^{-\kappa}\right)^{2}} \\
\quad \times \exp \left\{\frac{\beta \rho_{n}^{\prime}(0 \mid \alpha)}{\rho_{n}(0 \mid \alpha)}-\int_{\mathcal{C}_{n}} \frac{\mathrm{d} \lambda}{\pi \mathrm{i}} \lambda \partial_{\lambda} \ln \left(\frac{1+\mathfrak{a}_{n}(\lambda \mid \alpha)}{1+\mathfrak{a}_{0}(\lambda)}\right)\right. \\
\left.\quad+\int_{\mathcal{C}_{n}} \frac{\mathrm{d} \lambda}{2 \pi \mathrm{i}}\left[\frac{\Lambda_{n}^{\prime}(\lambda \mid \alpha)}{\Lambda_{n}(\lambda \mid \alpha)} \ln \left(1+\mathfrak{a}_{0}(\lambda)\right)-\frac{\Lambda_{0}^{\prime}(\lambda)}{\Lambda_{0}(\lambda)} \ln \left(1+\mathfrak{a}_{n}(\lambda \mid \alpha)\right)\right]\right\} .
\end{aligned}
$$

Note the similarity between the exponential term on the right hand side of this equation and the right hand side of equation (52). This similarity hints that a formula like (52) might also exist for the present case and that a simpler, more symmetric formula for $A_{n}^{-+}(\xi)$ can be derived.

There is indeed an alternative, less obvious formula for the form factor $F_{-}(\xi)$,

$$
\begin{gathered}
F_{-}(\xi)=\frac{\bar{G}_{-}^{+}(\xi)}{\left(q^{1+\alpha}-q^{-1-\alpha}\right)\left(q^{\alpha}-q^{-\alpha}\right)} \\
\frac{\mathrm{e}^{\xi} \prod_{j=1}^{M-1} q^{-\alpha} d\left(\mu_{j}\right) \mathrm{e}^{\mu_{j}}}{\prod_{j=1}^{M} \rho_{n}^{-1}\left(\lambda_{j} \mid \alpha\right) d\left(\lambda_{j}\right) \mathrm{e}^{\lambda_{j}}} \frac{\operatorname{det}_{M}\left\{\delta_{k}^{j}-\frac{\rho_{n}^{-1}\left(\lambda_{j} \mid \alpha\right)}{\mathfrak{a}_{0}^{\prime}\left(\lambda_{j} \mid \alpha\right)} K_{1+\alpha}\left(\lambda_{j}-\lambda_{k}\right)\right\}}{\operatorname{det}_{M}\left\{\delta_{k}^{j}-\frac{1}{\mathfrak{a}_{0}^{\prime}\left(\lambda_{j} \mid \alpha\right)} K\left(\lambda_{j}-\lambda_{k}\right)\right\}},
\end{gathered}
$$

where

$$
\bar{G}_{-}^{+}(\xi)=\lim _{\operatorname{Re} \lambda \rightarrow \infty} \bar{G}_{-}(\lambda, \xi)
$$


and $\bar{G}_{-}(\lambda, \xi)$ is the solution of the linear integral equation

$$
\begin{aligned}
\bar{G}_{-}(\lambda, \xi) & =-\operatorname{cth}(\lambda-\xi) \\
& +q^{\alpha+1} \rho_{n}^{-1}(\xi \mid \alpha) \operatorname{cth}(\lambda-\xi-\eta)+\int_{\mathcal{C}_{n}} \mathrm{~d} m_{-}^{\alpha}(\mu) \bar{G}_{-}(\mu, \xi) K_{1+\alpha}(\mu-\lambda) .
\end{aligned}
$$

The derivation is shown in appendix $B$.

Combining (55) and 61) we obtain a more symmetric expression for the amplitudes $A_{n}^{-+}(\xi)$,

$$
\begin{aligned}
& A_{n}^{-+}(\xi)=\frac{G_{+}^{-}(\xi) \bar{G}_{-}^{+}(\xi)}{\left(q^{1+\alpha}-q^{-1-\alpha}\right)\left(q^{\alpha}-q^{-\alpha}\right)} \frac{\prod_{j=1}^{M} \rho_{n}\left(\lambda_{j} \mid \alpha\right)}{\prod_{j=1}^{M-1} \rho_{n}\left(\mu_{j} \mid \alpha\right)} \\
& \times \frac{\operatorname{det}_{M-1}\left\{\delta_{k}^{j}-\frac{\rho_{n}\left(\mu_{j} \mid \alpha\right)}{\mathfrak{a}_{n}^{\prime}\left(\mu_{j} \mid \alpha\right)} K_{1-\alpha}\left(\mu_{j}-\mu_{k}\right)\right\}}{\operatorname{det}_{M-1}\left\{\delta_{k}^{j}-\frac{1}{\mathfrak{a}_{n}^{\prime}\left(\mu_{j} \mid \alpha\right)} K\left(\mu_{j}-\mu_{k}\right)\right\}} \frac{\operatorname{det}_{M}\left\{\delta_{k}^{j}-\frac{\rho_{n}^{-1}\left(\lambda_{j} \mid \alpha\right)}{\mathfrak{a}_{0}^{\prime}\left(\lambda_{j} \mid \alpha\right)} K_{1+\alpha}\left(\lambda_{j}-\lambda_{k}\right)\right\}}{\operatorname{det}_{M}\left\{\delta_{k}^{j}-\frac{1}{\mathfrak{a}_{0}^{\prime}\left(\lambda_{j} \mid \alpha\right)} K\left(\lambda_{j}-\lambda_{k}\right)\right\}} .
\end{aligned}
$$

In the Trotter limit it turns into

$$
\begin{aligned}
A_{n}^{-+}(\xi)=\frac{q^{\alpha+\kappa-1}+q^{-\alpha-\kappa+1}}{\left(q^{1+\alpha}-q^{-1-\alpha}\right)\left(q^{\alpha}-q^{-\alpha}\right)\left(q^{\kappa}+q^{-\kappa}\right)} & \\
& \times G_{+}^{-}(\xi) \bar{G}_{-}^{+}(\xi) \exp \left\{\int_{\mathcal{C}_{n}} \frac{\mathrm{d} \lambda}{2 \pi \mathrm{i}} \frac{\rho_{n}^{\prime}(\lambda \mid \alpha)}{\rho_{n}(\lambda \mid \alpha)} \ln \left(\frac{1+\mathfrak{a}_{n}(\lambda \mid \alpha)}{1+\mathfrak{a}_{0}(\lambda)}\right)\right\} \\
& \times \frac{\operatorname{det}_{\mathrm{d} m_{+}^{\alpha}, \mathcal{e}_{n}}\left\{1-\widehat{K}_{1-\alpha}\right\} \operatorname{det}_{\mathrm{d} m_{-}^{\alpha}, \mathfrak{e}_{n}}\left\{1-\widehat{K}_{1+\alpha}\right\}}{\operatorname{det}_{\mathrm{d} m_{0}^{\alpha}, \mathcal{C}_{n}}\{1-\widehat{K}\} \operatorname{det}_{\mathrm{d} m, \mathfrak{C}_{n}}\{1-\widehat{K}\}} .
\end{aligned}
$$

What we find remarkable about this expression is that it is basically of the same form as the expression (48) for the amplitudes in the form factor expansion of the generating function of the $z z$-correlation functions. The exponential term and the ratio of the products of two determinants appear in both cases, and the prefactor is a product of two functions satisfying 'conjugate' linear integral equations. All expressions are parameterized by the functions $\rho_{n}$ and $\mathfrak{a}_{n}$ which depend upon the specific excitation under consideration and are, of course, different in both cases. In appendix B we argue that $\lim _{\alpha \rightarrow 0} \bar{G}_{-}^{+}(\xi)=0$, such that the prefactor in 65 is non-singular in this limit.

Following [8,9] we can introduce 'the spin' of a local operator as the eigenvalue of the $z$-component of the operator of the total spin when acting adjointly on it. Then $\sigma^{-}$, $\sigma^{z}, \sigma^{+}$have eigenvalues $s=-, 0,+$, and we can interpret the parameterization of the kernels in the Fredholm determinants in the numerators of 48 and 65 as $\widehat{K}_{s-\alpha}$ and $\widehat{K}_{\alpha-s}$. In this combination the spin also appears in the $T Q$ equation.

\section{Generating function at high temperatures}

We are convinced that our formula for the amplitudes are not only aesthetically appealing but that they will also prove to be efficient for the future analytical and numerical analysis of the long-distance asymptotic behaviour of the finite-temperature correlation functions of the XXZ chain. In this and in the following section, we shall support this 
claim with two examples concerning the high- and the low-temperature asymptotics of the generating function of the longitudinal correlation function.

First of all, we use (48) to calculate the leading high-temperature contribution to the generating function $\left\langle q^{2 \alpha S(m)}\right\rangle$. Since the next-to leading eigenvalues vanish in the high-temperature limit, we only have to consider the first term $n=0$ of the expansion (30),

$$
\left\langle q^{2 \alpha S(m)}\right\rangle=A_{0}(\alpha) \rho_{0}^{m}(0 \mid \alpha)+\mathcal{O}\left(1 / T^{m}\right) .
$$

As is well known (see e.g. [55]), the dominant eigenvalue of the (twisted) quantum transfer matrix and the corresponding auxiliary function can be expanded in a power series in $1 / T$. Let us briefly review this procedure.

It follows from 21 with $M=N / 2$ that

$$
\mathfrak{a}_{0}(\lambda \mid \alpha)=q^{-2 \alpha}
$$

if $\beta=\kappa=0$. In order to calculate the next order, we assume the asymptotic expansion

$$
\mathfrak{a}_{0}(\lambda \mid \alpha)=q^{-2 \alpha}\left(1+\sum_{k=1}^{\infty} \frac{a_{k}(\lambda \mid \alpha)}{T^{k}}\right)
$$

By substituting (68) into (21) and expanding in powers of $1 / T$, we see that $a_{1}(\lambda \mid \alpha)$ is the solution of the linear integral equation

$$
a_{1}(\lambda \mid \alpha)=-h-2 J \operatorname{sh}(\eta) \mathrm{e}(\lambda)-\int_{\mathcal{C}_{0}} \frac{\mathrm{d} \mu}{2 \pi i} K(\lambda-\mu) \frac{a_{1}(\mu \mid \alpha)}{1+q^{2 \alpha}} .
$$

Obviously, the function

$$
a_{1}(\lambda \mid \alpha)=-h-2 J \operatorname{sh}(\eta)\left(\mathrm{e}(\lambda)-\frac{K(\lambda)}{1+q^{2 \alpha}}\right)
$$

is a solution of (69). Since $\mathfrak{a}_{0}(\lambda)=\mathfrak{a}_{0}(\lambda \mid 0)$, the corresponding expression for $\mathfrak{a}_{0}(\lambda)$ can be obtained from (70) by setting $\alpha=0$. These results can be substituted into (24), after evaluating the contour integrals we arrive at

$$
\rho_{0}(\lambda \mid \alpha)=\frac{q^{\alpha}+q^{-\alpha}}{2}\left(1+\frac{h}{2 T} \frac{q^{\alpha}-q^{-\alpha}}{q^{\alpha}+q^{-\alpha}}+\frac{J \operatorname{sh}(\eta)}{2 T}\left(\frac{q^{\alpha}-q^{-\alpha}}{q^{\alpha}+q^{-\alpha}}\right)^{2} K(\lambda)\right)+\mathcal{O}\left(1 / T^{2}\right) .
$$

By the same reasoning, we obtain

$$
\bar{\sigma}_{+}^{-} \sigma_{-}^{-}=1+\mathcal{O}\left(1 / T^{2}\right), \quad \exp \left\{\int_{\mathfrak{C}_{0}} \frac{\mathrm{d} \lambda}{2 \pi \mathrm{i}} \frac{\rho_{0}^{\prime}(\lambda \mid \alpha)}{\rho_{0}(\lambda \mid \alpha)} \ln \left(\frac{1+\mathfrak{a}_{0}(\lambda \mid \alpha)}{1+\mathfrak{a}_{0}(\lambda)}\right)\right\}=1+\mathcal{O}\left(1 / T^{2}\right) .
$$

Lastly, we have to consider the Fredholm determinants appearing in (48). Since every pole of the measure comes with a factor $1 / T$, we observe that the $k$-th term of the series (43) is of the order $\mathcal{O}\left(1 / T^{k}\right)$. Hence, for our purpose, it is sufficient to calculate the first term of the expansion (43). Using (70) and (71) we obtain after some calculations

$$
A_{0}(\alpha)=1+\frac{J \Delta}{T}\left(\frac{q^{\alpha}-q^{-\alpha}}{q^{\alpha}+q^{-\alpha}}\right)^{2}+\mathcal{O}\left(1 / T^{2}\right)
$$


Combining (71) and (73) with (66), we end up with

$$
\begin{aligned}
\left\langle q^{2 \alpha S(m)}\right\rangle= & \left(\frac{q^{\alpha}+q^{-\alpha}}{2}\right)^{m}+\frac{m h}{2 T}\left(\frac{q^{\alpha}-q^{-\alpha}}{2}\right)\left(\frac{q^{\alpha}+q^{-\alpha}}{2}\right)^{m-1} \\
& -(m-1) \frac{J \Delta}{T}\left(\frac{q^{\alpha}-q^{-\alpha}}{2}\right)^{2}\left(\frac{q^{\alpha}+q^{-\alpha}}{2}\right)^{m-2}+\mathcal{O}\left(1 / T^{2}\right) .
\end{aligned}
$$

Note that this result was already obtained in [45] within the multiple-integral approach to correlation functions.

Using 26] we can calculate the longitudinal correlators,

$$
\begin{aligned}
\left\langle\sigma_{1}^{z} \sigma_{2}^{z}\right\rangle & =-\frac{J \Delta}{T}+\mathcal{O}\left(1 / T^{2}\right), \\
\left\langle\sigma_{1}^{z} \sigma_{m+1}^{z}\right\rangle & =\mathcal{O}\left(1 / T^{2}\right), \quad m \geq 2 .
\end{aligned}
$$

The emptiness formation probability is given by

$$
\left\langle e_{1_{+}^{+}}^{+} \ldots e_{m+}^{+}\right\rangle=\left(\frac{1}{2}\right)^{m}\left(1+\frac{m h}{2 T}-(m-1) \frac{J \Delta}{T}\right)+\mathcal{O}\left(1 / T^{2}\right) .
$$

Note that (74), (75) and (76) are valid for all $\Delta>-1$. It will be interesting to extend the high-temperature expansion systematically to higher orders.

\section{Generating function at low temperatures}

The low-temperature limits of the massless and the massive regimes $(|\Delta| \leq 1$ and $\Delta>1$, respectively) have to be analyzed separately. For $\Delta>1$ the spectrum of the quantum transfer matrix remains gapped as $T \rightarrow 0$, but for $|\Delta|<1$ it becomes gapless, and the long-distance asymptotics of the static correlation functions is expected to be described by conformal field theory. Here we consider the massless case at finite magnetic field, $h>0$. The low-energy (large-distance) physics of our spin chain has then much in common with the physics of free Fermions. It is largely determined by particle-hole like excitations around the Fermi edge. As we shall see, the low-temperature analysis can be based on a generalization of the Sommerfeld expansion [50] to the interacting case. Such an analysis was partially carried out in [34], but here we have to go further and include the quantum numbers of the excited states.

Since the spectrum becomes gapless for $T \rightarrow 0$, infinitely many terms in the form factor expansion contribute to the large-distance asymptotics of the correlation functions. As we shall see, each individual contribution vanishes as a fractional power of the temperature. The situation is very similar as in case of the so-called critical form factors defined between eigenstates of the ordinary transfer matrix [25] which vanish in the thermodynamic limit as negative fractional powers of the system size. We shall also observe a close analogy of our result with that obtained for the Bose gas by one of the authors [37, 38] using a different method. As in [37] we will utilize a remarkable summation formula, obtained in [21, 24, 40], which allows us to sum up the leading low-temperature terms. This is necessary since the limit involved in the infinite series of the leading terms and the limit $T \rightarrow 0$ do not commute. 
The technique we are going to apply can be used for analyzing both, the longitudinal as well as the transversal correlation functions at small temperatures. Here we shall restrict ourselves to the analysis of the generating function of the longitudinal correlation functions and postpone the transversal case to a future publication. We start from the form factor expansion 30 . In order to determine the leading low-temperature behaviour of the amplitudes and eigenvalue ratios we use (45) and (24). The full calculation is rather lengthy. It consists of the following steps.

(i) Straightening of the contours in the non-linear integral equations 21 and in the expressions for the amplitudes (45) and eigenvalue ratios (24).

(ii) Low-temperature analysis of the non-linear integral equations.

(iii) Low-temperature analysis of the eigenvalue ratios.

(iv) Low-temperature analysis of the amplitudes.

(v) Summation of the leading terms.

Fortunately, the most sophisticated step (v) can be adopted from [24], and essentially only steps (i)-(iv) remain to be done.

\subsection{Straightening the contours}

The contour $\mathcal{C}_{0}$ consists of two straight lines parallel to the real axis and intersecting the imaginary axis at $\pm \eta / 2$ such that the real axis is 'encircled' in counterclockwise direction. The contours $\mathcal{C}_{n}$ are deformations of $\mathcal{C}_{0}$ such that all Bethe roots are included and all holes are excluded. To straighten $\mathcal{C}_{n}$ means to deform it into $\mathcal{C}_{0}$. In the process of this deformation the particle and hole parameters are crossed and the driving terms in the non-linear integral equations are supplemented by terms depending explicitly on these parameters.

We denote the number of hole parameters by $n_{h}$ and the number of particle parameters by $n_{p}$, set $n^{\prime}=n_{h}+n_{p}$ and define the functions

$$
E(\lambda)=\ln \left(\frac{\operatorname{sh}(\lambda)}{\operatorname{sh}(\eta+\lambda)}\right), \quad \theta(\lambda)=\ln \left(\frac{\operatorname{sh}(\eta-\lambda)}{\operatorname{sh}(\eta+\lambda)}\right)
$$

which are antiderivatives of the bare energy e $(\lambda)$ and of the kernel $K(\lambda)$. We further define

$$
z(\lambda)=-\frac{1}{2 \pi \mathrm{i}} \ln \left(\frac{1+\mathfrak{a}_{n}(\lambda \mid \alpha)}{1+\mathfrak{a}_{0}(\lambda)}\right) .
$$

A straightening of the contours in 21] leads to

$$
\begin{aligned}
\ln \left(\mathfrak{a}_{n}(\lambda)\right)= & \mathrm{i} \pi\left(n_{h}-n_{p}\right)-\left(\kappa+N / 2-M-n_{h}+n_{p}\right) 2 \eta-\beta \mathrm{e}(\lambda) \\
& +\sum_{j=1}^{n_{h}} \theta\left(\lambda-\lambda_{j}^{h}\right)-\sum_{j=1}^{n_{p}} \theta\left(\lambda-\lambda_{j}^{p}\right)-\int_{\mathfrak{C}_{0}} \frac{\mathrm{d} \mu}{2 \pi \mathrm{i}} K(\lambda-\mu) \ln \left(1+\mathfrak{a}_{n}(\mu)\right) .
\end{aligned}
$$

This defines an $n^{\prime}$-parametric family of functions, depending on $\left\{\lambda_{j}^{h}\right\}$ and $\left\{\lambda_{j}^{p}\right\}$. The individual functions $\mathfrak{a}_{n}$ are then determined by the subsidiary conditions

$$
\mathfrak{a}_{n}\left(\lambda_{j}^{h}\right)=\mathfrak{a}_{n}\left(\lambda_{k}^{p}\right)=-1, \quad j=1, \ldots, n_{h}, \quad k=1, \ldots, n_{p}
$$


fixing these parameters to a discrete set of values.

We assume that for sufficiently small temperature

$$
N / 2-M-n_{h}+n_{p}=0
$$

and use this condition in our low-temperature analysis without further mentioning. Then the eigenvalue ratios take the form

$$
\rho_{n}(0 \mid \alpha)=q^{\alpha} \exp \left\{\sum_{j=1}^{n_{h}} E\left(\lambda_{j}^{h}\right)-\sum_{j=1}^{n_{p}} E\left(\lambda_{j}^{p}\right)-\int_{\mathcal{C}_{0}} \mathrm{~d} \lambda \mathrm{e}(\lambda) z(\lambda)\right\}
$$

The amplitudes (45) consist of a determinant factor times

$$
A_{n}^{(0)}(\alpha)=\exp \left\{\int_{\mathcal{C}_{n}} \frac{\mathrm{d} \lambda}{2 \pi \mathrm{i}} \frac{\rho_{n}^{\prime}(\lambda \mid \alpha)}{\rho_{n}(\lambda \mid \alpha)} \ln \left(\frac{1+\mathfrak{a}_{n}(\lambda \mid \alpha)}{1+\mathfrak{a}_{0}(\lambda)}\right)\right\} .
$$

The determinant factor is treated in appendix D. Inserting (24) into (83) and straightening the contours we obtain

$$
\begin{aligned}
A_{n}^{(0)}(\alpha) & =\exp \left\{-\int_{\mathcal{C}_{0}} \mathrm{~d} \lambda \int_{\mathcal{C}_{0}^{\prime}} \mathrm{d} \mu z(\lambda) \mathrm{e}^{\prime}(\lambda-\mu) z(\mu)\right\} \\
& \times \exp \left\{2 \int_{\mathcal{C}_{0}} \mathrm{~d} \lambda z(\lambda)\left[\sum_{j=1}^{n_{p}} \operatorname{cth}\left(\lambda-\lambda_{j}^{p}\right)-\sum_{j=1}^{n_{h}} \operatorname{cth}\left(\lambda-\lambda_{j}^{h}\right)\right]\right\} \\
& \times \exp \left\{-\int_{\mathcal{C}_{0}} \mathrm{~d} \lambda z(\lambda)\left[\sum_{j=1}^{n_{p}}\left(\operatorname{cth}\left(\lambda-\lambda_{j}^{p}+\eta\right)+\operatorname{cth}\left(\lambda-\lambda_{j}^{p}-\eta\right)\right)\right.\right. \\
\times & {\left[\prod_{j=1}^{n_{p}}\left(\partial_{\lambda} \mathrm{e}^{-2 \pi \mathrm{i} z}(\lambda)\right)_{\lambda=\lambda_{j}^{p}}^{-1}\right]\left[\prod_{j=1}^{n_{h}}\left(\partial_{\lambda} \mathrm{e}^{-2 \pi \mathrm{i} z(\lambda)}\right)_{\lambda=\lambda_{j}^{h}}^{-1}\right] } \\
\times & \frac{\left[\prod_{\substack{n_{h}=1 \\
j \neq k}}^{n_{h}} \operatorname{sh}\left(\lambda_{j}^{h}-\lambda_{k}^{h}\right)\right]\left[\prod_{\substack{n_{p} \\
j \neq 1}}^{n_{j}} \operatorname{sh}\left(\lambda_{j}^{p}-\lambda_{k}^{p}\right)\right]}{\left[\prod_{j=1}^{n_{h}} \prod_{k=1}^{n_{p}} \operatorname{sh}\left(\lambda_{j}^{h}-\lambda_{k}^{p}\right)\right]^{2}} \\
\times & \frac{\prod_{j=1}^{n_{h}} \prod_{k=1}^{n_{p}} \operatorname{sh}\left(\lambda_{j}^{h}-\lambda_{k}^{p}+\eta\right) \operatorname{sh}\left(\lambda_{j}^{h}-\lambda_{k}^{p}-\eta\right)}{\left[\prod_{j, k=1}^{n_{h}} \operatorname{sh}\left(\lambda_{j}^{h}-\lambda_{k}^{h}-\eta\right)\right]\left[\prod_{j, k=1}^{n_{p}} \operatorname{sh}\left(\lambda_{j}^{p}-\lambda_{k}^{p}+\eta\right)\right]} .
\end{aligned}
$$

Here $\mathrm{C}_{0}^{\prime}$ is a contour infinitesimally close to $\mathrm{C}_{0}$ and inside $\mathcal{C}_{0}$. The equation holds for $N=2 M$. For $N \neq 2 M$ additional phase factors may arise.

\subsection{Low-temperature analysis of the non-linear integral equations}

From now on we restrict ourselves to the computation of quantities related to the generating function. Then $M=N / 2$. We introduce

$$
\varepsilon_{0}(\lambda)=h-\frac{4 J\left(1-\Delta^{2}\right)}{\operatorname{ch}(2 \lambda)-\Delta}
$$


and

$$
u_{0}(\lambda)=-T \ln \left(\mathfrak{a}_{0}(\lambda+\mathrm{i} \gamma / 2)\right), \quad u(\lambda)=-T \ln \left(\mathfrak{a}_{n}(\lambda+\mathrm{i} \gamma / 2 \mid \alpha)\right),
$$

where we suppressed the index $n$ and the dependence on $\alpha$ in the definition of $u$.

It follows from 797 that

$$
\begin{aligned}
u(\lambda)=\varepsilon_{0}(\lambda)+T\left[2 \pi \mathrm{i} \alpha^{\prime}+\sum_{j=1}^{n_{p}} \theta(\lambda-\right. & \left.\left.\lambda_{j}^{p}+\mathrm{i} \gamma / 2\right)-\sum_{j=1}^{n_{h}} \theta\left(\lambda-\lambda_{j}^{h}+\mathrm{i} \gamma / 2\right)\right] \\
& +T \int_{\mathcal{C}_{0}-\mathrm{i} \gamma / 2} \frac{\mathrm{d} \mu}{2 \pi \mathrm{i}} K(\lambda-\mu) \ln \left(1+\mathrm{e}^{-\frac{u(\mu)}{T}}\right),
\end{aligned}
$$

where we introduced $\alpha^{\prime}=\eta \alpha / \mathrm{i} \pi$. A similar equation without the contribution proportional to $T$ in the driving term holds for $u_{0}$,

$$
u_{0}(\lambda)=\varepsilon_{0}(\lambda)+T \int_{\mathcal{C}_{0}-\mathrm{i} \gamma / 2} \frac{\mathrm{d} \mu}{2 \pi \mathrm{i}} K(\lambda-\mu) \ln \left(1+\mathrm{e}^{-\frac{u_{0}(\mu)}{T}}\right) .
$$

Since $\theta$ is bounded on the contour $\mathcal{C}_{0}-\mathrm{i} \gamma$, the terms in square brackets in (87) may be neglected compared to $\varepsilon_{0}(\lambda)$ when $T$ becomes small. Thus, $u$ and $u_{0}$ have the same zero temperature limit $\varepsilon$. The integrals in (87) and (88) vanish on those parts of the contour on which $\operatorname{Re} \varepsilon>0$ and are nonzero on their complement. We claim that this complement is an interval $[-Q, Q]$ on the real axis. Indeed, if $\varepsilon$ satisfies the linear integral equation

$$
\varepsilon(\lambda)=\varepsilon_{0}(\lambda)+\int_{-Q}^{Q} \frac{\mathrm{d} \mu}{2 \pi \mathrm{i}} K(\lambda-\mu) \varepsilon(\mu),
$$

where $Q$ is determined by the condition $\varepsilon( \pm Q)=0$, then $\varepsilon$ behaves similarly as the driving term. It is real and even on $\mathbb{R}$, has at most the two zeros $\pm Q$ and is negative on $[-Q, Q]$. One can prove that $\operatorname{Re} \varepsilon>h / 4>0$ for all $\gamma \in(0, \pi / 2)$ on $\mathbb{R}-\mathrm{i} \gamma$, which is the lower part of the integration contour in (87), 88). Hence, $\varepsilon$ satisfies (87) and (88) up to corrections at most of the order of $T$. The function $\varepsilon$ is the well-known dressed energy function.

Our argument becomes rigorous by employing the following 'generalized Sommerfeld lemma' which also allows us to obtain the first and second order temperature corrections below.

Lemma 1. Let let $u, f$ be holomorphic in an open set containing a contour $\mathrm{C}_{u}$, and let f be bounded on $\mathrm{C}_{u}$. Let $v=\operatorname{Re} u, w=\operatorname{Im} u$. Assume that $v$ has exactly two zeros $Q_{ \pm}$ on $\mathrm{C}_{u}$ separating $\mathcal{C}_{u}$ into a part $\mathfrak{C}_{u}^{-}$between $Q_{-}$and $Q_{+}$on which $v$ is negative and a remainder $\mathcal{C}_{u}^{+}$on which $v$ is positive. Assume that $\exists p \in \mathbb{Z}$ such that $w\left(Q_{ \pm}\right)=2 \pi p T$. Assume that $\mathrm{C}_{u}$ is oriented in such a way that $Q_{-}$comes before $Q_{+}$on $\mathrm{C}_{u}^{-}$. Then (for $T>0)$

$$
\begin{aligned}
T \int_{\mathcal{C}_{u}} \mathrm{~d} \lambda f(\lambda) \ln \left(1+\mathrm{e}^{-\frac{u(\lambda)}{T}}\right)=-\int_{Q_{-}}^{Q_{+}} & \mathrm{d} \lambda f(\lambda)(u(\lambda)-2 \pi \mathrm{i} p T) \\
+ & \frac{T^{2} \pi^{2}}{6}\left(\frac{f\left(Q_{+}\right)}{u^{\prime}\left(Q_{+}\right)}-\frac{f\left(Q_{-}\right)}{u^{\prime}\left(Q_{-}\right)}\right)+\mathcal{O}\left(T^{4}\right) .
\end{aligned}
$$


We present a proof of this lemma in appendix C. The lemma is designed in such a way that it can be directly applied to the integral equations (87), (88). For small $T$ the functions $u$ and $u_{0}$ are close to $\varepsilon$ and therefore satisfy the requirements of Lemma 1 . Hence,

$$
\begin{aligned}
\bar{u}(\lambda)=\varepsilon_{0}(\lambda)+\operatorname{Tr}_{1}(\lambda)+\frac{\mathrm{i} \pi T^{2}}{12}\left[\frac{K\left(\lambda-Q_{+}\right)}{u^{\prime}\left(Q_{+}\right)}\right. & \left.-\frac{K\left(\lambda-Q_{-}\right)}{u^{\prime}\left(Q_{-}\right)}\right] \\
& +\int_{Q_{-}}^{Q_{+}} \frac{\mathrm{d} \mu}{2 \pi \mathrm{i}} K(\lambda-\mu) \bar{u}(\mu)+\mathcal{O}\left(T^{4}\right),
\end{aligned}
$$

where $\bar{u}(\lambda)=u(\lambda)-2 \pi \mathrm{i} p T$ and

$$
r_{1}(\lambda)=2 \pi \mathrm{i}\left(\alpha^{\prime}-p\right)+\sum_{j=1}^{n_{p}} \theta\left(\lambda-\lambda_{j}^{p}+\mathrm{i} \gamma / 2\right)-\sum_{j=1}^{n_{h}} \theta\left(\lambda-\lambda_{j}^{h}+\mathrm{i} \gamma / 2\right)
$$

An equation similar to (91) holds for $u_{0}$.

If we neglect the $\mathcal{O}\left(T^{4}\right)$ terms, equation 91 is already a linear integral equation for $\bar{u}$. In the next step we resolve the $T$-dependence of $Q_{ \pm}$. We make the ansatz

$$
u(\lambda)=\varepsilon(\lambda)+T u_{1}(\lambda)+T^{2} u_{2}(\lambda)+\mathcal{O}\left(T^{3}\right), \quad Q_{ \pm}= \pm Q+T Q_{ \pm}^{(1)}+\mathcal{O}\left(T^{2}\right)
$$

Using that $u\left(Q_{ \pm}\right)=2 \pi \mathrm{i} p T$ and $\varepsilon( \pm Q)=0$ we immediately obtain

$$
Q_{ \pm}= \pm Q \mp \frac{\bar{u}_{1}( \pm Q)}{\varepsilon^{\prime}(Q)} T+\mathcal{O}\left(T^{2}\right)
$$

where we agree upon $\bar{u}_{1}(\lambda)=u_{1}(\lambda)-2 \pi \mathrm{i} p$.

Inserting (94) into (91) and expanding up to the second order in $T$ we obtain

$$
\bar{u}(\lambda)=\varepsilon_{0}(\lambda)+\operatorname{Tr}_{1}(\lambda)+T^{2} r_{2}(\lambda)+\int_{-Q}^{Q} \frac{\mathrm{d} \mu}{2 \pi \mathrm{i}} K(\lambda-\mu) \bar{u}(\mu)+\mathcal{O}\left(T^{3}\right),
$$

where

$$
r_{2}(\lambda)=\frac{\mathrm{i} \pi}{4 \varepsilon^{\prime}(Q)}\left[K(\lambda-Q)\left(\frac{1}{3}+\frac{\bar{u}_{1}^{2}(Q)}{\pi^{2}}\right)+K(\lambda+Q)\left(\frac{1}{3}+\frac{\bar{u}_{1}^{2}(-Q)}{\pi^{2}}\right)\right] .
$$

Then the linearity of 95 implies that $\bar{u}_{1}$ and $u_{2}$ satisfy

$$
\begin{aligned}
& \bar{u}_{1}(\lambda)=r_{1}(\lambda)+\int_{-Q}^{Q} \frac{\mathrm{d} \mu}{2 \pi \mathrm{i}} K(\lambda-\mu) \bar{u}_{1}(\mu), \\
& u_{2}(\lambda)=r_{2}(\lambda)+\int_{-Q}^{Q} \frac{\mathrm{d} \mu}{2 \pi \mathrm{i}} K(\lambda-\mu) u_{2}(\mu) .
\end{aligned}
$$

We can express these functions by means of a set of standard functions that appear in the description of the ground state properties of the model. We introduce the dressed charge function $Z$, the root density function $\rho$, the dressed phase $\phi$ and the resolvent $R$ 
as solutions of the linear integral equations

$$
\begin{aligned}
Z(\lambda) & =1+\int_{-Q}^{Q} \frac{\mathrm{d} \mu}{2 \pi \mathrm{i}} K(\lambda-\mu) Z(\mu), \\
\rho(\lambda) & =-\frac{\mathrm{e}(\lambda+\mathrm{i} \gamma / 2)}{2 \pi \mathrm{i}}+\int_{-Q}^{Q} \frac{\mathrm{d} \mu}{2 \pi \mathrm{i}} K(\lambda-\mu) \rho(\mu), \\
\phi(\lambda, v) & =-\frac{\theta(\lambda-\mathrm{v})}{2 \pi \mathrm{i}}+\int_{-Q}^{Q} \frac{\mathrm{d} \mu}{2 \pi \mathrm{i}} K(\lambda-\mu) \phi(\mu, v), \\
R(\lambda, v) & =K(\lambda-v)+\int_{-Q}^{Q} \frac{\mathrm{d} \mu}{2 \pi \mathrm{i}} K(\lambda-\mu) R(\mu, v) .
\end{aligned}
$$

Comparing (97) and 98) we conclude that

$$
\begin{aligned}
& \bar{u}_{1}(\lambda)=2 \pi \mathrm{i}\left[\left(\alpha^{\prime}-p\right) Z(\lambda)+\sum_{j=1}^{n_{h}} \phi\left(\lambda, \lambda_{j}^{h}-\mathrm{i} \gamma / 2\right)-\sum_{j=1}^{n_{p}} \phi\left(\lambda, \lambda_{j}^{p}-\mathrm{i} \gamma / 2\right)\right], \\
& u_{2}(\lambda)=\frac{\mathrm{i} \pi}{4 \varepsilon^{\prime}(Q)}\left[R(\lambda, Q)\left(\frac{1}{3}+\frac{\bar{u}_{1}^{2}(Q)}{\pi^{2}}\right)+R(\lambda,-Q)\left(\frac{1}{3}+\frac{\bar{u}_{1}^{2}(-Q)}{\pi^{2}}\right)\right] .
\end{aligned}
$$

The root density $\rho$ will reappear later when we calculate the low-temperature asymptotics of the correlation lengths. Recall that $\ln \left(\mathfrak{a}_{n}(\lambda+\mathrm{i} \gamma / 2 \mid \alpha)\right)=-\varepsilon(\lambda) / T-u_{1}(\lambda)-$ $u_{2}(\lambda) T+\mathcal{O}\left(T^{2}\right)$. Thus, we have obtained the solutions of 79 for $M=N / 2$ and $n_{p}=n_{h}$ up to corrections of order $T^{2}$.

It remains to determine the hole and particle parameters $\lambda_{j}^{h}$ and $\lambda_{k}^{p}$ by means of the subsidiary condition 80 . In terms of the function $\bar{u}$ the latter reads

$$
\bar{u}\left(\lambda_{j}^{h}-\mathrm{i} \gamma / 2\right)=2 \pi \mathrm{i} T\left(h_{j}-1 / 2\right), \quad \bar{u}\left(\lambda_{k}^{p}-\mathrm{i} \gamma / 2\right)=2 \pi \mathrm{i} T\left(p_{k}-1 / 2\right),
$$

where $h_{j}, p_{k} \in \mathbb{Z}$ are such that the $\lambda_{j}^{h}$ are located below $\mathcal{C}_{0}$ while the $\lambda_{k}^{p}$ are located above $\mathcal{C}_{0}$. Assuming that the quantum numbers $h_{j}, p_{k}$ of the particles and holes are all different and that the $\lambda_{j}^{h}$ and $\lambda_{k}^{p}$ are uniquely determined by 100 we may write

$$
\lambda_{j}^{h}=\mathrm{i} \gamma / 2+x_{h_{j}}, \quad \lambda_{k}^{p}=\mathrm{i} \gamma / 2+y_{p_{k}} .
$$

We restrict ourselves to excitations close to the Fermi edges $\pm Q$ for which

$$
x_{h_{j}}^{ \pm}= \pm Q+\mathcal{O}(T), \quad y_{p_{k}}^{ \pm}= \pm Q+\mathcal{O}(T)
$$

and denote the number of holes and particles close to the left and right Fermi edge by $n_{h}^{-}, n_{p}^{-}$and $n_{h}^{+}, n_{p}^{+}$, respectively. Since we assume that $n_{h}=n_{p}=n^{\prime}$ we have

$$
n_{h}^{-}-n_{p}^{-}=n_{p}^{+}-n_{h}^{+}=\ell .
$$

Using (102), 103 and the identity $\phi(\lambda, Q)-\phi(\lambda,-Q)=Z(\lambda)-1$, following from 98a, 98c, we infer that

$$
\bar{u}_{1}(\lambda)=\bar{u}_{1}^{(\ell)}(\lambda)+\mathcal{O}(T), \quad \bar{u}_{1}^{(\ell)}(\lambda)=2 \pi \mathrm{i}\left(\left(\alpha^{\prime}-\ell-p\right) Z(\lambda)+\ell\right) .
$$

Hence,

$$
\begin{aligned}
& \bar{u}\left(x_{h_{j}}^{ \pm}\right)=\mp 2 \pi \mathrm{i} T\left(h_{j}^{ \pm}-1 / 2\right)= \pm \varepsilon^{\prime}(Q)\left(x_{h_{j}}^{ \pm} \mp Q\right)+\bar{u}_{1}^{(\ell)}(Q) T+\mathcal{O}\left(T^{2}\right), \\
& \bar{u}\left(y_{p_{k}}^{ \pm}\right)= \pm 2 \pi \mathrm{i} T\left(p_{k}^{ \pm}-1 / 2\right)= \pm \varepsilon^{\prime}(Q)\left(y_{p_{k}}^{ \pm} \mp Q\right)+\bar{u}_{1}^{(\ell)}(Q) T+\mathcal{O}\left(T^{2}\right),
\end{aligned}
$$


where the signs in the first equations have to be understood as part of the definitions of $h_{j}^{ \pm}$and $p_{k}^{ \pm}$. It follows that

$$
\begin{aligned}
& x_{h_{j}}^{ \pm}=Q_{ \pm}-\frac{2 \pi \mathrm{i} T}{\varepsilon^{\prime}(Q)}\left(h_{j}^{ \pm}-1 / 2\right)+\mathcal{O}\left(T^{2}\right), \\
& y_{p_{k}}^{ \pm}=Q_{ \pm}+\frac{2 \pi \mathrm{i} T}{\mathcal{\varepsilon}^{\prime}(Q)}\left(p_{k}^{ \pm}-1 / 2\right)+\mathcal{O}\left(T^{2}\right) .
\end{aligned}
$$

Our definition of particles and holes implies that

$$
\operatorname{Im} x_{h_{j}}^{ \pm}<0, \quad \operatorname{Im} y_{p_{k}}^{ \pm}>0 .
$$

Equation (106) together with the constraint 107) determines the roots of the function $1+\mathfrak{a}_{n}(\lambda \mid \alpha)$ close to the Fermi points to first order in $T$. To this order they are equidistantly located on two parallel lines intersecting the canonical contour $\mathcal{C}_{0}$ at a right angle in $\mathrm{i} \gamma / 2 \mp Q$. These roots can be occupied by particles or holes. The picture is very much the same as for free Fermions. The parameter $\ell$, introduced in $(103)$, is the excess of particles over holes at the right Fermi edge which, in conventional language, is describing the 'number of Umklapp processes' involved in the excitation. The analogy with free Fermions (the case $\Delta=0$ ) also implies that $p$ is the number of roots below $Q_{+}$if $Q_{+}$is above $\mathcal{C}_{0}$ (minus the number of roots between $Q_{+}$and $\mathrm{C}_{0}$ if $Q_{+}$is below). For $p \neq 0$ we cannot naively place particles or holes between $Q_{ \pm}$and $\mathfrak{C}_{0}$, since this would be inconsistent with our derivation. For $p \neq 0$ we have to take into account that we might cross particle or hole parameters in the course of deforming the contour $\mathcal{C}_{0}$ into the contour $\mathcal{C}_{u}$ required by Lemma 1 . In this case the function $r_{1}$ in (92) has to be modified accordingly. In the following we shall avoid such complication by simply assuming that $p=0$. We shall see that this is already enough to derive the leading large-distance asymptotics of the longitudinal correlation functions for small temperatures and shall come back to the general case in a forthcoming publication.

Since $p=0$ by hypothesis, we suppress the bar in $\bar{u}, \bar{u}_{1}$ etc. in the following except in some general statements that are valid for any $p \in \mathbb{Z}$. Setting $p=0$ means that the points $Q_{ \pm}+\mathrm{i} \gamma / 2$ are located between the roots closest to $\mathcal{C}_{0}$, whence $\left|\operatorname{Im} u_{1}^{(\ell)}(Q)\right|<\pi$. Using (104) with $p=0$ and setting $z=Z(Q)$ this condition is equivalent to

$$
-\frac{\operatorname{Re} \alpha^{\prime}}{z^{-1}-1}-\frac{1}{2(1-z)}<\ell<-\frac{\operatorname{Re} \alpha^{\prime}}{z^{-1}-1}+\frac{1}{2(1-z)}
$$

The latter inequality restricts the range of $\ell$ for given $\alpha^{\prime}, z$. It is easy to see that $1 / \sqrt{2}<z<1$ for $0<\Delta<1$. Thus, $\sqrt{108}$ ) is always satisfied for $\ell=-1,0,1$ as long as $\operatorname{Re} \alpha^{\prime}$ is small enough. As we shall see, these values determine the leading largedistance asymptotics of the correlation functions. Obviously the inequality (108) can be satisfied by any $\ell \in \mathbb{Z}$ if $z$ is close enough to 1 or if we choose $\alpha^{\prime}$ appropriately. For this reason we keep $\ell \in \mathbb{Z}$ general in our subsequent analysis.

For the low-temperature analysis of the eigenvalue ratios in the next subsection we need $u_{1}^{(\ell)}$ up to the first order in $T$. We insert 104, 106 into 99a and use 
$\partial_{\nu} \phi(\lambda, v)=R(\lambda, v) / 2 \pi$ i to obtain

$$
\begin{aligned}
u_{1}(\lambda)= & u_{1}^{(\ell)}(\lambda)+\frac{2 \pi \mathrm{i} T}{\varepsilon^{\prime}(Q)}\left\{R(\lambda, Q)\left[\frac{\ell u_{1}^{(\ell)}(Q)}{2 \pi \mathrm{i}}-\sum_{j=1}^{n_{h}^{+}}\left(h_{j}^{+}-\frac{1}{2}\right)-\sum_{j=1}^{n_{p}^{+}}\left(p_{j}^{+}-\frac{1}{2}\right)\right]\right. \\
& \left.+R(\lambda,-Q)\left[\frac{\ell u_{1}^{(\ell)}(Q)}{2 \pi \mathrm{i}}-\sum_{j=1}^{n_{h}^{-}}\left(h_{j}^{-}-\frac{1}{2}\right)-\sum_{j=1}^{n_{p}^{-}}\left(p_{j}^{-}-\frac{1}{2}\right)\right]\right\}+\mathcal{O}\left(T^{2}\right) .
\end{aligned}
$$

Equations (99b) and (109) together with the corresponding linear integral equations then determine $\ln \left(\mathfrak{a}_{n}(\lambda \mid \alpha)\right)$ up to the order $T$. Using these equations we can readily calculate the leading order low-temperature expansion of the eigenvalue ratios.

\subsection{Low-temperature analysis of the eigenvalue ratios}

We start from the representation 82 of the eigenvalue ratio. In order to expand the sums in the exponent we use (106). Then

$$
\begin{aligned}
\sum_{j=1}^{n_{h}} E\left(\lambda_{j}^{h}\right)-\sum_{j=1}^{n_{p}} E\left(\lambda_{j}^{p}\right)=-2 \ell E(Q+\mathrm{i} \gamma / 2) & \\
& +\frac{2 \pi \mathrm{i} T \mathrm{e}(Q+\mathrm{i} \gamma / 2)}{\varepsilon^{\prime}(Q)}\left[\frac{\ell u_{1}^{(\ell)}(Q)}{\mathrm{i} \pi}-\sum_{j=1}^{n^{\prime}}\left(h_{j}+p_{j}-1\right)\right]+\mathcal{O}\left(T^{2}\right) .
\end{aligned}
$$

It remains to calculate the integral

$$
-\int_{\mathfrak{C}_{0}} \mathrm{~d} \lambda \mathrm{e}(\lambda) z(\lambda)=\int_{\mathfrak{C}_{0}-\mathrm{i} \gamma / 2} \frac{\mathrm{d} \lambda}{2 \pi \mathrm{i}} \mathrm{e}(\lambda+\mathrm{i} \gamma / 2) \ln \left(\frac{1+\mathrm{e}^{-\frac{u(\lambda)}{T}}}{1+\mathrm{e}^{-\frac{u_{0}(\lambda)}{T}}}\right) .
$$

For this purpose one can employ Lemma 1 as well as the formulae of the previous section. Introducing the Fermi momentum

$$
k_{F}=\pi \int_{-Q}^{Q} \mathrm{~d} \lambda \rho(\lambda)
$$

and the sound velocity

$$
v_{0}=\frac{\varepsilon^{\prime}(Q)}{2 \pi \rho(Q)}
$$

we obtain altogether

$$
\begin{aligned}
\rho_{n}(0 \mid \alpha)=q^{\alpha} \exp \{ & -2 \mathrm{i}\left(\alpha^{\prime}-\ell\right) k_{F} \\
- & \left.\frac{2 \pi T}{v_{0}}\left[\left(\alpha^{\prime}-\ell\right)^{2} z^{2}-\ell^{2}+\sum_{j=1}^{n^{\prime}}\left(h_{j}+p_{j}-1\right)\right]\right\}+\mathcal{O}\left(T^{2}\right) .
\end{aligned}
$$




\subsection{Low-temperature analysis of the amplitudes}

The low-temperature analysis of the amplitudes $A_{n}^{(0)}(\alpha)$, eqn. 84 , is the most tedious part of the calculation. Those terms in the expression for the amplitudes that depend explicitly on the hole and particle parameters can be expanded using (106). We obtain for the respective leading terms

$$
\begin{aligned}
& {\left[\prod_{j=1}^{n^{\prime}}\left(\partial_{\lambda} \mathrm{e}^{-2 \pi \mathrm{i} z(\lambda)}\right)_{\lambda=\lambda_{j}^{p}}^{-1}\left(\partial_{\lambda} \mathrm{e}^{-2 \pi \mathrm{i} z(\lambda)}\right)_{\lambda=\lambda_{j}^{h}}^{-1}=(-1)^{\ell}\left(\frac{T}{\varepsilon^{\prime}(Q)}\right)^{2 n^{\prime}}\left(1-\mathrm{e}^{u_{1}^{(\ell)}(Q)}\right)^{2 n^{\prime}},\right.} \\
& {\left[\prod_{\substack{j, k=1 \\
j \neq k}}^{n^{\prime}} \operatorname{sh}\left(\lambda_{j}^{h}-\lambda_{k}^{h}\right) \operatorname{sh}\left(\lambda_{j}^{p}-\lambda_{k}^{p}\right)\right]\left[\prod_{j, k=1}^{n^{\prime}} \operatorname{sh}\left(\lambda_{j}^{h}-\lambda_{k}^{p}\right)\right]^{-2}} \\
& \quad=\left(\frac{2 \pi \mathrm{i} T}{\varepsilon^{\prime}(Q)}\right)^{2 \ell^{2}-2 n^{\prime}} \operatorname{sh}^{-2 \ell^{2}}(2 Q) \prod_{\varepsilon= \pm} \frac{\prod_{1 \leq j<k \leq n_{h}^{\varepsilon}}\left(h_{j}^{\varepsilon}-h_{k}^{\varepsilon}\right)^{2} \prod_{1 \leq j<k \leq n_{p}^{\varepsilon}}\left(p_{j}^{\varepsilon}-p_{k}^{\varepsilon}\right)^{2}}{\prod_{j=1}^{n_{h}^{\varepsilon}} \prod_{k=1}^{n_{p}^{\varepsilon}}\left(h_{j}^{\varepsilon}+p_{k}^{\varepsilon}-1\right)^{2}}, \\
& \prod_{j, k=1}^{n^{\prime}} \frac{\operatorname{sh}\left(\lambda_{j}^{h}-\lambda_{k}^{p}+\eta\right) \operatorname{sh}\left(\lambda_{j}^{h}-\lambda_{k}^{p}-\eta\right)}{\operatorname{sh}\left(\lambda_{j}^{h}-\lambda_{k}^{h}-\eta\right) \operatorname{sh}\left(\lambda_{j}^{p}-\lambda_{k}^{p}+\eta\right)}=\left(\frac{\operatorname{sh}(\eta+2 Q) \operatorname{sh}(\eta-2 Q)}{\operatorname{sh}^{2}(\eta)}\right)^{\ell^{2}} .
\end{aligned}
$$

The integrals in the exponent in (84) which have a non-singular integrand can be evaluated by means of Lemma 1 which together with 94 implies the following

Corollary 1. For $f$ holomorphic in a finite strip around $\mathrm{C}_{0}$ and $z$ defined in (78)

$$
\int_{\mathcal{C}_{0}} \mathrm{~d} \lambda f(\lambda) z(\lambda)=-\int_{-Q}^{Q} \frac{\mathrm{d} \lambda}{2 \pi \mathrm{i}} f(\lambda+\mathrm{i} \gamma / 2) \bar{u}_{1}^{(\ell)}(\lambda)+\mathcal{O}(T) .
$$

This can be used to conclude that to leading order in $T$

$$
\begin{aligned}
& -\int_{\mathcal{C}_{0}} \mathrm{~d} \lambda z(\lambda)\left[\sum_{j=1}^{n^{\prime}}\left(\operatorname{cth}\left(\lambda-\lambda_{j}^{p}+\eta\right)+\operatorname{cth}\left(\lambda-\lambda_{j}^{p}-\eta\right)\right)\right. \\
& \left.-\sum_{j=1}^{n^{\prime}}\left(\operatorname{cth}\left(\lambda-\lambda_{j}^{h}+\eta\right)+\operatorname{cth}\left(\lambda-\lambda_{j}^{h}-\eta\right)\right)\right] \\
& \quad+\int_{\mathcal{C}_{0}} \mathrm{~d} \lambda \int_{\mathcal{C}_{0}^{\prime}} \mathrm{d} \mu z(\lambda) \operatorname{cth}^{\prime}(\lambda-\mu+\eta) z(\mu) \\
& =\ell^{2} \ln \left(\frac{\operatorname{sh}^{2}(\eta)}{\operatorname{sh}(\eta+2 Q) \operatorname{sh}(\eta-2 Q)}\right)-\left(\alpha^{\prime}-\ell\right)^{2} \int_{-Q}^{Q} \mathrm{~d} \lambda \int_{-Q}^{Q} \mathrm{~d} \mu \frac{Z(\lambda) Z(\mu)}{\operatorname{sh}^{2}(\lambda-\mu+\eta)}
\end{aligned}
$$

The remaining integrals involve singularities close to the contour of integration and are harder to estimate. Fortunately these integrals are similar to integrals that appeared previously in the calculation of the low-temperature asymptotics of the generating function of the density-density correlators of the Bose gas in [37]. The following lemma is slightly stronger than the corresponding statement in case of the Bose gas in appendix B of [37] and can be obtained in the same spirit.

Lemma 2. Let $u$ and $\mathcal{C}_{u}$ be subject to the same assumptions as in Lemma 7 Let $\lambda_{+}$be located above $\mathrm{C}_{u}$ and $\lambda_{-}$below. Then the Cauchy-type integral

$$
I_{u}\left(\lambda_{ \pm}\right)=\int_{\mathcal{C}_{u}} \mathrm{~d} \lambda \operatorname{cth}\left(\lambda-\lambda_{ \pm}\right) \ln \left(1+\mathrm{e}^{-\frac{u(\lambda)}{T}}\right)
$$


admits a low-temperature expansion whose form depends on the distance of $\lambda_{ \pm}$from the zeros $Q_{ \pm}$of the real part of $u$.

For $\delta>0$ define $V_{ \pm}=\left\{z \in \mathbb{C}|| u(z) \mid<\delta / 2, z\right.$ close to $\left.Q_{ \pm}\right\}$. If $\lambda_{ \pm}$are uniformly away from $Q_{ \pm}$, then Lemma 1 applies, and

$$
I_{u}\left(\lambda_{ \pm}\right)=-\int_{Q_{-}}^{Q_{+}} \mathrm{d} \lambda \operatorname{cth}\left(\lambda-\lambda_{ \pm}\right) \frac{\bar{u}(\lambda)}{T}+\mathcal{O}(T) .
$$

If $\lambda_{ \pm} \in V_{+}$, then

$$
\begin{array}{r}
I_{u}\left(\lambda_{ \pm}\right)=-\int_{Q_{-}}^{Q_{+}} \mathrm{d} \lambda \operatorname{cth}\left(\lambda-\lambda_{ \pm}\right) \frac{\bar{u}(\lambda)-\bar{u}\left(\lambda_{ \pm}\right)}{T} \mp 2 \pi \mathrm{i} \ln \left\{\Gamma\left(\frac{1}{2} \pm \frac{\bar{u}\left(\lambda_{ \pm}\right)}{2 \pi \mathrm{i} T}\right)\right\} \\
\pm \pi \mathrm{i} \ln (2 \pi)+\frac{\bar{u}\left(\lambda_{ \pm}\right)}{T}\left\{\ln \left(\frac{\bar{u}\left(\lambda_{ \pm}\right)}{ \pm 2 \pi \mathrm{i} T}\right)-1-\ln \left(\frac{\operatorname{sh}\left(Q_{+}-\lambda_{ \pm}\right)}{\operatorname{sh}\left(Q_{-}-\lambda_{ \pm}\right)}\right)\right\}+\mathcal{O}(T) .
\end{array}
$$

If $\lambda_{ \pm} \in V_{-}$, then

$$
\begin{array}{r}
I_{u}\left(\lambda_{ \pm}\right)=-\int_{Q_{-}}^{Q_{+}} \mathrm{d} \lambda \operatorname{cth}\left(\lambda-\lambda_{ \pm}\right) \frac{\bar{u}(\lambda)-\bar{u}\left(\lambda_{ \pm}\right)}{T} \mp 2 \pi \mathrm{i} \ln \left\{\Gamma\left(\frac{1}{2} \mp \frac{\bar{u}\left(\lambda_{ \pm}\right)}{2 \pi \mathrm{i} T}\right)\right\} \\
\pm \pi \mathrm{i} \ln (2 \pi)-\frac{\bar{u}\left(\lambda_{ \pm}\right)}{T}\left\{\ln \left(\frac{\bar{u}\left(\lambda_{ \pm}\right)}{\mp 2 \pi \mathrm{i} T}\right)-1+\ln \left(\frac{\operatorname{sh}\left(Q_{+}-\lambda_{ \pm}\right)}{\operatorname{sh}\left(Q_{-}-\lambda_{ \pm}\right)}\right)\right\}+\mathcal{O}(T) .
\end{array}
$$

First of all this can be used to calculate the periodic Cauchy transform

$$
L_{\mathfrak{C}_{0}}[z](v)=\int_{\mathfrak{C}_{0}} \mathrm{~d} \mu z(\mu) \operatorname{cth}(\mu-v)
$$

when $v$ is close to $i \gamma / 2 \pm Q$. Assuming this and setting $s=\operatorname{sign}(\operatorname{Im}(\lambda))$ we find

$$
\begin{aligned}
& L_{\mathcal{C}_{0}}[z](\lambda+\mathrm{i} \gamma / 2)=-\int_{-Q}^{Q} \frac{\mathrm{d} \mu}{2 \pi \mathrm{i}} \operatorname{cth}(\mu-\lambda)\left(u_{1}(\mu)-u_{1}(\lambda)\right) \\
& +\frac{u_{1}(\lambda)}{2 \pi \mathrm{i}} \ln \left(\frac{\varepsilon^{ \pm 1}(\lambda) \operatorname{sh}(\lambda+Q)}{\operatorname{sh}(\lambda-Q)}\right) \mp \frac{u_{1}(\lambda)}{2 \pi \mathrm{i}} \ln ( \pm s 2 \pi \mathrm{i} T) \\
& \quad-s \ln \left(\frac{\Gamma(1 / 2 \pm s u(\lambda) / 2 \pi \mathrm{i} T)}{\Gamma\left(1 / 2 \pm s u_{0}(\lambda) / 2 \pi \mathrm{i} T\right)}\right)+\mathcal{O}(T) .
\end{aligned}
$$

This formula is useful for the evaluation of the remaining integrals. Using also 105a and (106) we obtain

$$
\begin{aligned}
2 \int_{\mathcal{C}_{0}} \mathrm{~d} \lambda z(\lambda)\left[\sum_{j=1}^{n^{\prime}} \operatorname{cth}\left(\lambda-\lambda_{j}^{p}\right)-\sum_{j=1}^{n^{\prime}} \operatorname{cth}\left(\lambda-\lambda_{j}^{h}\right)\right] \\
=\frac{2 \ell u_{1}^{(\ell)}(Q)}{\pi \mathrm{i}} \ln \left(\frac{\varepsilon^{\prime}(Q) \operatorname{sh}(2 Q)}{2 \pi T}\right)-n^{\prime} u_{1}^{(\ell)}(Q) \\
\quad-4 \ell \int_{-Q}^{Q} \frac{\mathrm{d} \lambda}{2 \pi \mathrm{i}}\left(u_{1}^{(\ell)}(\lambda)-u_{1}^{(\ell)}(Q)\right) \operatorname{cth}(\lambda-Q)
\end{aligned}
$$




$$
\begin{aligned}
+\ln \{ & {\left[\prod_{j=1}^{n_{h}^{+}} \frac{\Gamma^{2}\left(h_{j}^{+}+\frac{u_{1}^{(\ell)}(Q)}{2 \pi \mathrm{i}}\right)}{\Gamma^{2}\left(h_{j}^{+}\right)}\right]\left[\prod_{j=1}^{n_{p}^{+}} \frac{\Gamma^{2}\left(p_{j}^{+}-\frac{u_{1}^{(\ell)}(Q)}{2 \pi \mathrm{i}}\right)}{\Gamma^{2}\left(p_{j}^{+}\right)}\right] } \\
& \left.\times\left[\prod_{j=1}^{n_{h}^{-}} \frac{\Gamma^{2}\left(h_{j}^{-}-\frac{u_{1}^{(\ell)}(Q)}{2 \pi \mathrm{i}}\right)}{\Gamma^{2}\left(h_{j}^{-}\right)}\right]\left[\prod_{j=1}^{n_{p}^{-}} \frac{\Gamma^{2}\left(p_{j}^{-}+\frac{u_{1}^{(\ell)}(Q)}{2 \pi \mathrm{i}}\right)}{\Gamma^{2}\left(p_{j}^{-}\right)}\right]\right\}+\mathcal{O}(T \ln (T)) .
\end{aligned}
$$

Finally the most delicate term is the remaining double integral with singular integrand. For this integral we can use again (123) and proceed analogously to appendix B of [37]. Then to leading order in $T$

$$
\begin{aligned}
-\int_{\mathrm{C}_{0}} \mathrm{~d} \lambda \int_{\mathfrak{C}_{0}^{\prime}} \mathrm{d} \mu z(\lambda) \operatorname{cth}^{\prime}(\lambda-\mu) z(\mu)=2\left(\frac{u_{1}^{(\ell)}(Q)}{2 \pi \mathrm{i}}\right)^{2} \ln \left(\frac{2 \pi T}{\varepsilon^{\prime}(Q) \operatorname{sh}(2 Q)}\right) \\
+2 \ln \left\{G\left(1-\frac{u_{1}^{(\ell)}(Q)}{2 \pi \mathrm{i}}\right) G\left(1+\frac{u_{1}^{(\ell)}(Q)}{2 \pi \mathrm{i}}\right)\right\}+C_{1}\left[\frac{u_{1}^{(\ell)}}{2 \pi \mathrm{i}}\right]
\end{aligned}
$$

where $G$ is the Barnes $G$-function and $C_{1}$ is a functional defined by

$$
C_{1}[v]=\frac{1}{2} \int_{-Q}^{Q} \mathrm{~d} \lambda \int_{-Q}^{Q} \mathrm{~d} \mu \frac{v^{\prime}(\lambda) v(\mu)-v(\lambda) v^{\prime}(\mu)}{\operatorname{th}(\lambda-\mu)}+2 v(Q) \int_{-Q}^{Q} \mathrm{~d} \lambda \frac{v(\lambda)-v(Q)}{\operatorname{th}(\lambda-Q)}
$$

When we insert all the terms (115), (117), (124) and (125) into (84) and also use 104 they neatly combine. The amplitude $A_{n}^{(0)}$ decomposes into two factors $A_{n}^{( \pm)}$ pertaining to the left and right Fermi edge:

$$
A_{n}^{(0)}(\mathrm{i} \pi \alpha / \eta)=A_{n}^{(-)}(\alpha) A_{n}^{(+)}(\alpha)
$$

where

$$
\begin{aligned}
A_{n}^{(\varepsilon)}(\alpha)=G^{2}(1 & -\varepsilon[\ell+(\alpha-\ell) z])\left(\frac{2 \pi T \mathrm{e}^{C[Z / z]}}{\varepsilon^{\prime}(Q) \operatorname{sh}(2 Q)}\right)^{(\alpha-\ell)^{2} z^{2}} \\
& \times\left(\frac{\sin (\pi(\alpha-\ell) z)}{\pi}\right)^{2 n_{h}^{\varepsilon}} \mathcal{R}_{n_{h}^{\varepsilon}, n_{p}^{\varepsilon}}\left(\left\{h_{j}^{\varepsilon}\right\},\left\{p_{j}^{\varepsilon}\right\} \mid \varepsilon[(\alpha-\ell) z+\ell]\right),
\end{aligned}
$$

for $\varepsilon= \pm 1$, and where we have introduced the shorthand notations

$$
C[v]=\frac{1}{2} \int_{-Q}^{Q} \mathrm{~d} \lambda \int_{-Q}^{Q} \mathrm{~d} \mu\left[\frac{v^{\prime}(\lambda)(v(\mu)+1)-v^{\prime}(\mu)(v(\lambda)+1)}{2 \operatorname{th}(\lambda-\mu)}-\frac{v(\lambda) v(\mu)}{\operatorname{sh}^{2}(\lambda-\mu+\eta)}\right]
$$

and

$$
\begin{aligned}
\mathcal{R}_{n_{1}, n_{2}}\left(\left\{h_{j}\right\},\left\{p_{j}\right\} \mid v\right)=\frac{\prod_{1 \leq j<k \leq n_{1}}\left(h_{j}-h_{k}\right)^{2} \prod_{1 \leq j<k \leq n_{2}}\left(p_{j}-p_{k}\right)^{2}}{\prod_{j=1}^{n_{1}} \prod_{k=1}^{n_{2}}\left(h_{j}+p_{k}-1\right)^{2}} \\
\times\left[\prod_{j=1}^{n_{1}} \frac{\Gamma^{2}\left(h_{j}+v\right)}{\Gamma^{2}\left(h_{j}\right)}\right]\left[\prod_{j=1}^{n_{2}} \frac{\Gamma^{2}\left(p_{j}-v\right)}{\Gamma^{2}\left(p_{j}\right)}\right] .
\end{aligned}
$$


Thus, in the low-temperature limit we have have obtained an entirely explicit description of the 'universal contributions' $A_{n}^{(0)}$ to the amplitudes in terms of the dressed charge and the dressed energy. Let us recall that the above formulae (128)-(130) are valid for a certain class of excitations characterized by (i) $n_{p}=n_{h}$, and (ii) $x_{h_{j}}^{ \pm}, y_{p_{j}}^{ \pm}= \pm Q+\mathcal{O}(T)$.

The Fredholm determinant part of the amplitudes is a fraction with two Fredholm determinants in the numerator and two Fredholm determinants in the denominator. In order to calculate the zero temperature limit of the determinants in the denominator we recall that the weight functions $\left(1+\mathfrak{a}_{0}^{-1}(\lambda)\right)^{-1}$ and $\left(1+\mathfrak{a}_{n}^{-1}(\lambda \mid \alpha)\right)^{-1}$ play the role of the Fermi functions for our system. In the zero temperature limit they turn into the characteristic functions of the 'interval' $\mathrm{i} \gamma / 2+[-Q, Q]$ on $\mathrm{C}_{0}$. Thus,

$$
\lim _{T \rightarrow 0+} \operatorname{det}_{\mathrm{d} m_{0}^{\alpha}, \mathrm{e}_{n}}\{1-\widehat{\mathcal{K}}\}=\lim _{T \rightarrow 0+} \operatorname{det}_{\mathrm{d} m, \mathrm{e}_{n}}\{1-\widehat{\mathcal{K}}\}=\operatorname{det}_{\frac{\mathrm{d} \lambda}{2 \pi},[-Q, Q]}\{1-\widehat{\mathcal{K}}\} .
$$

The expression on the right hand side depends only on the anisotropy parameter and on the magnetic field. For the proof we note the estimate [41]

$$
|\operatorname{det}(1-A)-\operatorname{det}(1-B)| \leq\|A-B\|_{1} \exp \left(\|A\|_{1}+\|B\|_{1}+1\right),
$$

where $\|A\|_{1}=\operatorname{tr}|A|$ and which is valid for trace class operators $A, B$ [42].

The zero temperature limit of the determinants in the numerator is more technical. In order to obtain it we need to assume certain general properties of the low-temperature behaviour of zeroes of certain functions (see appendix D). Setting

$$
\mathrm{d} \widehat{M}_{ \pm}^{\alpha}(\lambda)=\frac{\mathrm{d} \lambda}{2 \pi \mathrm{i}} \frac{\mathrm{e}^{ \pm \mathrm{i} \pi \alpha^{\prime} \pm\left(\alpha^{\prime}-\ell\right) \int_{-Q}^{Q} \mathrm{~d} \mu \mathrm{e}(\mu-\lambda) Z(\mu)}}{1-\mathrm{e}^{ \pm 2 \pi \mathrm{i}\left(\alpha^{\prime}-\ell\right) Z(\lambda)}}
$$

and denoting by $\Gamma[-Q, Q]$ a contour which encircles the interval $[-Q, Q]$ counterclockwise we conjecture that

$$
\lim _{T \rightarrow 0+\mathrm{d} m_{ \pm}^{\alpha}, e_{n}}\left\{1-\widehat{\mathcal{K}}_{\mp \alpha}\right\}=\operatorname{det}_{\mathrm{d} \widehat{M}_{ \pm}^{\alpha}, \Gamma[-Q, Q]}\left\{1-\widehat{\mathcal{K}}_{\mp \alpha}\right\} .
$$

With (131), (134) the zero temperature limit of the determinant part of the amplitude is

$$
\mathcal{D}(\ell)=\frac{\operatorname{det}_{\mathrm{d} \widehat{M}_{+}^{\alpha}, \Gamma[-Q, Q]}\left\{1-\widehat{\mathcal{K}}_{-\alpha}\right\} \operatorname{det}_{\mathrm{d}_{\widehat{M}_{-}^{\alpha}, \Gamma[-Q, Q]}^{\alpha}\left\{1-\widehat{\mathcal{K}}_{\alpha}\right\}}}{\operatorname{det}_{\frac{\mathrm{d} \lambda}{2 \pi \mathrm{i}},[-Q, Q]}^{2}\{1-\widehat{\mathcal{K}}\}} .
$$

The important point about this expression is that it depends only on $\ell$ but not on the 'quantum numbers' $p_{j}, h_{k}$ of the particle hole excitations.

In appendix $\mathrm{D}$ we justify the above conjecture. We also provide alternative expressions for the ratios of Fredholm determinants that allow us to compare our formulae with those obtained for zero temperature in [25] and to extract the $\alpha$ dependence from the determinants. Moreover, we provide expressions in which ratios of determinants are replaced by determinants of a different type of operators.

\subsection{Summation of the leading terms}

As we already mentioned above it is possible to sum up the form factors of particle-hole type considered in the previous subsections. Summation is possible for each individual 
value of $\ell$ by means of the same summation formula as employed in [24] in the context of the so-called critical form factors pertaining to the eigenstates of the ordinary transfer matrix. Adapted to our notation it reads

$$
\begin{array}{r}
\sum_{\substack{n_{p}, n_{h} \geq 0 \\
n_{p}-n_{h}=\ell}} \sum_{\substack{p_{1}<\cdots<p_{n_{p}} \\
p_{j} \in \mathbb{N}}} \sum_{\substack{h_{1}<\cdots<h_{h_{h}} \\
h_{j} \in \mathbb{N}}} \mathrm{e}^{-\frac{2 \pi m T}{v_{0}}\left[\sum_{j=1}^{n_{p}}\left(p_{j}-1\right)+\sum_{j=1}^{n_{h}} h_{j}\right]}\left(\frac{\sin (\pi v)}{\pi}\right)^{2 n_{h}} R_{n_{h}, n_{p}}\left(\left\{h_{j}\right\},\left\{p_{j}\right\} \mid v\right) \\
=\frac{G^{2}(1+\ell-v)}{G^{2}(1-v)} \frac{\mathrm{e}^{-\frac{\pi m T \ell(\ell-1)}{v_{0}}}}{\left(1-\mathrm{e}^{-\frac{2 \pi m T}{v_{0}}}\right)^{(\ell-v)^{2}}} \cdot \quad
\end{array}
$$

Summing the contributions to the form factors series of the form considered in the previous subsections amounts to summing over all sets of mutually distinct quantum numbers $\left\{h_{j}^{+}\right\},\left\{p_{k}^{+}\right\}$and $\left\{h_{j}^{-}\right\},\left\{p_{k}^{-}\right\}$under the constraint $n_{p}^{+}-n_{h}^{+}=n_{h}^{-}-n_{p}^{-}=\ell$ and then summing over the allowed values of $\ell \in \mathbb{Z}$. Using (136) we end up with

$$
\left\langle\mathrm{e}^{2 \pi \mathrm{i} \alpha S(m)}\right\rangle \sim \sum_{\ell} \mathrm{e}^{\mathrm{i} \pi \alpha m+2 \mathrm{i} k_{F}(\ell-\alpha) m} \mathcal{D}(\ell) \mathcal{A}(\ell-\alpha)\left(\frac{\pi T / v_{0}}{\operatorname{sh}\left(m \pi T / v_{0}\right)}\right)^{2(\ell-\alpha)^{2} z^{2}},
$$

where

$$
\mathcal{A}(x)=\frac{\mathrm{e}^{2 x^{2} C[Z / z] z^{2}} G^{2}(1+x z) G^{2}(1-x z)}{(2 \pi \rho(Q) \operatorname{sh}(2 Q))^{2 x^{2} z^{2}}} .
$$

For every finite temperature (137) is a sum over terms which decay exponentially with the distance $m$. For $T \rightarrow 0+$ the nature of the asymptotics changes from exponential to algebraic decay, $\left(\pi T / v_{0}\right) / \operatorname{sh}\left(m \pi T / v_{0}\right) \rightarrow 1 / m$, as is expected at a critical point. Up to an adjustment of conventions and notation (137) turns into the sum obtained in [24]. In particular, the amplitude $\mathcal{D}(\ell) \mathcal{A}(\ell-\alpha)$ is the same as in the zero temperature case.

Looking at it the other way round, the lowest order effect of switching on the temperature is a deformation, $1 / m \rightarrow\left(\pi T / v_{0}\right) / \operatorname{sh}\left(m \pi T / v_{0}\right)$, exactly as expected from conformal field theory by mapping the complex plane to a cylinder of finite circumference [10]. Here we have obtained this result directly from an expansion of the generating function in terms of form factors of the quantum transfer matrix.

The series (137) is neither an asymptotic series for small $T$ nor for large $m$. In [37] it was characterized as a sum gathering the 'leading orders for each oscillatory term.' This means that for each order of exponential decay we have neglected algebraic corrections in $T$ which would contribute lower order terms than the next-order exponentials. In addition, we have neglected higher order correction to the inverse correlation lengths that would amount to contributions of the form $\exp \left(\mathcal{O}\left(m T^{2}\right)\right)$. Thus, 137$)$ provides only the leading order asymptotics. On the other hand, each oscillating term appears with its coefficient of leading order in $T$.

Recall that we have assumed in our above analysis that $p=0$. This assumption restricts the allowed range of summation over $\ell$ for fixed $\alpha$ to the values determined by $(108)$. For any $\Delta \in(0,1)$ and any value of the magnetic field this range includes $\ell=-1,0,1$ if $\alpha$ is small enough. It becomes large for magnetic fields close to saturation and for anisotropies close to zero. We believe that an extension of our analysis to $p \neq 0$ will show that the sum may be extended to all $\ell \in \mathbb{Z}$. This would also restore the periodicity in $\alpha$ of the generating function. We shall come back to this in future work. 


\subsection{Large-distance asymptotics of the longitudinal correlation functions}

The large-distance asymptotics of the longitudinal correlation functions can be obtained from (137) by means of (26), (27). From (138) and from appendix D we know that

$$
\begin{array}{lrl}
\mathcal{D}(0) & =1+\mathcal{O}\left(\alpha^{2}\right), & \mathcal{D}(\ell)=\mathcal{O}\left(\alpha^{2}\right) \text { for } \ell \neq 0, \\
\mathcal{A}(0) & =1, & \mathcal{A}(\ell-\alpha)=\mathcal{A}(\ell)+\mathcal{O}(\alpha) \text { for } \ell \neq 0 .
\end{array}
$$

Then (27) implies

$$
\left\langle\sigma_{1}^{z}\right\rangle=1-\frac{2 k_{F}}{\pi}
$$

which is the known relation between magnetization and Fermi momentum. Using this formula and (26) we obtain to leading order (i.e. by taking into account only $\ell=-1,0,1$ in (137))

$$
\begin{aligned}
\left\langle\sigma_{1}^{z} \sigma_{m+1}^{z}\right\rangle-\left\langle\sigma_{1}^{z}\right\rangle\left\langle\sigma_{m+1}^{z}\right\rangle=-\frac{2 z^{2}}{\pi^{2}}\left(\frac{\pi T / v_{0}}{\operatorname{sh}\left(m \pi T / v_{0}\right)}\right)^{2} & \\
& +\frac{4 \sin ^{2}\left(k_{F}\right)}{\pi^{2}} \mathcal{A}(1) \mathcal{D}^{\prime \prime}(1) \cos \left(2 m k_{F}\right)\left(\frac{\pi T / v_{0}}{\operatorname{sh}\left(m \pi T / v_{0}\right)}\right)^{2 z^{2}} .
\end{aligned}
$$

Here we have introduced the shorthand notation $\mathcal{D}^{\prime \prime}(1)=\left.\partial_{\alpha}^{2} \mathcal{D}(1)\right|_{\alpha=0}$ and used that $\mathcal{D}^{\prime \prime}(-1)=\mathcal{D}^{\prime \prime}(1)$ and $\mathcal{A}(-1)=\mathcal{A}(1)$.

Depending on whether $Z>1$ or $Z<1$ either the first or the second term determines the large-distance asymptotics of the correlation function. It is easy to see from the integral equation (98a) for the dressed charge function that $z>1$ for $-1<\Delta<0$ and $z<1$ for $0<\Delta<1$ provided that $Q>0$. Thus, as is well known [1, 39], the lowtemperature correlation functions are asymptotically always negative if $-1<\Delta<0$, while they exhibit $2 k_{F}$ oscillations if $0<\Delta<1$. At the free Fermion point $\Delta=0$ both types of behaviour coexist. The amplitude in the first term is proportional to the square of the dressed charge and is numerically easily accessible. It was obtained using a perturbative scheme around $\Delta=0$ in [1]. The amplitude in the second term was apparently not known until recently [22]. It is not yet clear how well the Fredholm determinant part can be computed numerically. This question deserves further attention.

Note that we have derived (141) assuming that $0<\Delta<1$. It remains valid for $-1<\Delta<0$, since the correlation functions in this regime of the anisotropy can be obtained from those for $0<\Delta<1$ by a simple transformation [56] consisting of a sign reversal of the coupling $J$ and a conjugation of all local operators on even sites by $\sigma^{z}$.

\section{Ground state correlation functions of the finite chain}

The description of the spectral properties of integrable finite-length systems can be based on similar auxiliary functions as introduced above in order to deal with the distributions of Bethe roots of the quantum transfer matrix. In fact it is exactly this context in which auxiliary functions and non-linear integral equations first became relevant for integrable quantum systems [32]. As we have pointed out elsewhere [4, 12] the form of many formulae for correlation functions is the same for the infinite chain

at finite temperature and for the finite chain in the ground state with no magnetic 
field applied. Here we claim that this also holds for the formulae for the form factors and amplitudes obtained above. Moreover, if one is willing to accept slightly more complicated contours, then the finite-length case with non-zero magnetic field can be treated in a similar manner. The resulting formulae may be problematic for a numerical treatment, but we expect that the large- $L$ asymptotics may be analyzed in analogy with the low-temperature case in the previous section. As compared to the approach pursued in [23,25] we would keep control of the finite-size dependence at every stage of the calculation which might allow us, in principle, to obtain higher order finite-size corrections. Yet, since we have not carried out the calculation, we shall leave the realm of speculation at this point and simply describe how equations (48) and (65) for the amplitudes have to be modified.

In the finite-length case we work with the monodromy matrix

$$
T_{a}(\lambda)=q^{\kappa \sigma_{a}^{z}} R_{a, L}(\lambda, \eta / 2) \ldots R_{a,-L+1}(\lambda, \eta / 2)
$$

acting on the tensor product of an auxiliary space with index $a$ and $2 L$ quantum spaces. Here

$$
\kappa=\mathrm{i} \phi / \eta
$$

and $\phi$ is a real flux parameter.

The corresponding transfer matrix $t(\lambda)=\operatorname{Tr}_{a}\left\{T_{a}(\lambda)\right\}$ generates the Hamiltonian of the XXZ chain,

$$
H=2 J \operatorname{sh}(\eta) t^{-1}(\eta / 2) t^{\prime}(\eta / 2)=J \sum_{j=-L+1}^{L}\left(\sigma_{j-1}^{x} \sigma_{j}^{x}+\sigma_{j-1}^{y} \sigma_{j}^{y}+\Delta\left(\sigma_{j-1}^{z} \sigma_{j}^{z}-1\right)\right),
$$

with twisted periodic boundary conditions,

$$
\sigma_{-L}^{b}=q^{-\kappa \sigma_{L}^{z}} \sigma_{L}^{b} q^{\kappa \sigma_{L}^{z}}, \quad b=x, y, z
$$

Since $T_{a}(\lambda)$ satisfies the Yang-Baxter algebra relations $(6)$, the eigenstates and eigenvalues of $t(\lambda)$ can again be constructed by means of the algebraic Bethe ansatz. The only essential difference is in the pseudo vacuum, which in this case is $|0\rangle=\left(\begin{array}{l}1 \\ 0\end{array}\right)^{\otimes 2 L}$, and, consequently, in the vacuum expectation values $a(\lambda), d(\lambda)$ of the diagonal elements of the monodromy matrix which are now given by

$$
a(\lambda)=q^{\kappa}, \quad d(\lambda)=q^{-\kappa} b^{2 L}(\lambda, \eta / 2) .
$$

Inserting these vacuum expectation values into the Bethe equations (16) we obtain rather different patterns of roots in the complex plane. Still, the definitions (19) and (20) remain meaningful, and a non-linear integral equation similar to 21) can be derived for the auxiliary functions,

$$
\ln \left(\mathfrak{a}_{n}(\lambda)\right)=-2(\kappa-M) \eta+2 \mathrm{i} L \mathrm{p}(\lambda)-\int_{\mathfrak{C}_{n}} \frac{\mathrm{d} \mu}{2 \pi \mathrm{i}} K(\lambda-\mu) \ln \left(1+\mathfrak{a}_{n}(\mu)\right) .
$$

Here

$$
\mathrm{p}(\lambda)=\mathrm{i} \ln \left(\frac{\operatorname{sh}(\lambda+\eta / 2)}{\operatorname{sh}(\lambda-\eta / 2)}\right)
$$


is the bare momentum, whose appearance seems rather naturally in view of the fact that we have interchanged 'space and time direction' in the underlying six-vertex model. With the auxiliary functions (147) the formula (24) for the ratio of two transfer matrix eigenvalues remains valid in the finite-length case if we replace $N$ by $2 L$.

The general formula (12) for the correlation functions remains valid as well. Due our conventions for the monodromy matrix (142) the operators and eigenvalues on the right hand side have to be evaluated at $\eta / 2$ instead of 0 ,

$$
\left\langle\mathcal{O}_{1}^{(1)} \ldots \mathcal{O}_{m}^{(m)}\right\rangle=\frac{\left\langle\Psi_{0}\left|\operatorname{Tr}\left\{\mathcal{O}^{(1)} T(\eta / 2)\right\} \ldots \operatorname{Tr}\left\{\mathcal{O}^{(m)} T(\eta / 2)\right\}\right| \Psi_{0}\right\rangle}{\left\langle\Psi_{0} \mid \Psi_{0}\right\rangle \Lambda_{0}^{m}(\eta / 2)} .
$$

The corresponding form factor expansions of the generating function of the longitudinal correlation functions and of the transversal two-point function follow as

$$
\left\langle q^{2 \alpha S(m)}\right\rangle_{N}=\sum_{n=0}^{N_{M}-1} A_{n}(\alpha) \rho_{n}^{m}\left(\frac{\eta}{2} \mid \alpha\right), \quad\left\langle\sigma_{1}^{-} \sigma_{m+1}^{+}\right\rangle_{N}=\sum_{n=1}^{N_{M}} A_{n}^{-+}\left(\frac{\eta}{2}\right) \rho_{n}^{m}\left(\frac{\eta}{2} \mid \alpha\right) .
$$

Here the number of overturned spins $M$ must be $L$ for the generating function and $L-1$ for the transversal correlation function. Not only the structure of these asymptotic expansions is the same as in the finite-temperature case, but equations (48) and (65) for the amplitudes remain valid as well. Note, however, that in the derivation of the integral formula (20) for the ratio of the eigenvalues as well as in the derivation of the formula for the amplitudes in the asymptotic expansion of the transversal correlation functions we have assumed that the spectral parameter is located inside the integration contour. This must be kept in mind when sending it to $\eta / 2$. In order to deal with a finite magnetic field one has to switch to a sector of non-zero eigenvalue of the $z$-component of the total spin. $\Lambda_{0}$ must be replaced by the largest transfer matrix eigenvalue in this sector, and the contours have to be changed accordingly.

\section{Conclusions}

In this work we have initiated a study of the finite-temperature correlation functions of the XXZ chain by means of the form factors of the quantum transfer matrix. Products of two form factors combine into the amplitudes in the long-distance asymptotics expansions of the two-point correlation functions. These amplitudes seem to be more natural than the form factors themselves. There is no issue about their proper normalization, whereas the normalization we chose e.g. for $F_{-}(\xi)$ and $F_{+}(\xi)$ in 53 seems to be somewhat arbitrary.

For the amplitudes we were able to perform the Trotter limit analytically. The resulting formulae (48) and (65) for the longitudinal and transversal cases have a remarkably similar structure. They consist of a 'Fredholm determinant part', a 'universal amplitude part', which seems to carry the critical behaviour in the zero temperature limit, and a 'factorizing part' by which we mean the product of $\sigma$-functions in (48) or the product of $G$-functions in 65), respectively. We believe that such type of structure persists for more general form factors such as $\left\langle\Psi_{n}^{\alpha}\left|C\left(\xi_{1}\right) C\left(\xi_{2}\right)\right| \Psi_{0}\right\rangle$. This conjecture is supported by [2, 8, 19], where factorization of correlation functions was proved in a rather general context.

We have demonstrated that our formulae can be efficiently analyzed at least for low and high temperatures. This analysis will be continued in future work. We hope 
that the formulae for the amplitudes can also be evaluated numerically. At fixed finite temperature a few (typically one or two) amplitudes and the corresponding correlation lengths fix the leading asymptotics. Due to the efficiency of asymptotic expansions a combination with the exact short-distance results of [3, 44, 54] would give us numerical access to the full correlation functions and would allow us to calculate their Fourier transforms which appear in many applications in condensed matter physics.

An interesting feature of our Fredholm determinants is that they truncate exactly for every finite Trotter number (every finite length in the finite-length case). For finite Trotter number they are represented by finitely many integrals (see (41)). The Fredholm determinants as well as the other factors in the expressions for the amplitudes are parameterized by the auxiliary functions $\mathfrak{a}_{n}$ whose low-temperature behaviour is rather well known. In particular it can be also analyzed for vanishing magnetic field [31]. We hope that this fact will help us to analyze the low-temperature asymptotics of the full thermal correlation functions for zero magnetic field which is one of the many interesting open problems in our approach.

A further interesting issue to be studied in future work are finite-temperature singularities of the amplitudes which may occur e.g. at the cross-over temperatures [13] where the asymptotic behaviour of the correlation functions changes from decaying and oscillating to monotonically decaying. Finally, we would like to mention the possibility to study dynamical correlation functions at finite temperature by means of the form factors of the quantum transfer matrix. Results for zero temperature, based on the form factor approach, were recently obtained in [26].

Acknowledgment. The authors are grateful to H. Boos, N. Kitanine, A. Klümper and A. Weiße for helpful and encouraging discussions. MD and FG acknowledge financial support by the Volkswagen Foundation. KKK is supported by the CNRS. His work has been partly financed by the grant PEPS-PTI 'Asymptotique d'intégrales multiples' and by a Burgundy region PARI 2013 FABER grant 'Structures et asymptotiques d'intégrales multiples'. 


\section{Appendix A: Amplitudes of the longitudinal correlation functions}

In this appendix we sketch the derivation of the expression (38) for the amplitudes $A_{n}(\alpha)$ in the form factor expansion of the generating function of the longitudinal two-point functions defined in 30 .

Recall that for the longitudinal correlation functions only excited states of the twisted transfer matrix with $M=N / 2$ Bethe roots contribute to the form factor expansion. We denote these Bethe roots by $\mu_{j}, j=1, \ldots, M$, while the Bethe roots of the dominant state will be denoted $\lambda_{j}, j=1, \ldots, M$. Using the scalar product formula (36) we obtain

$$
\begin{aligned}
& {\left[\prod_{j=1}^{M} \frac{d\left(\mu_{j}\right)}{d\left(\lambda_{j}\right)}\right] \frac{\left\langle\Psi_{n}^{\alpha} \mid \Psi_{0}\right\rangle}{\left\langle\Psi_{n}^{\alpha} \mid \Psi_{n}^{\alpha}\right\rangle}=\left[\prod_{j=1}^{M}-\phi^{-1}\left(\mu_{k}-\eta\right)\right] } \\
& \times\left[\prod_{1 \leq j<k \leq M} \frac{\operatorname{sh}\left(\mu_{j}-\mu_{k}\right)}{\operatorname{sh}\left(\lambda_{j}-\lambda_{k}\right)}\right] \frac{\operatorname{det}_{M}\left\{\mathrm{e}\left(\lambda_{j}-\mu_{k}\right)-\mathrm{e}\left(\mu_{k}-\lambda_{j}\right) \mathfrak{a}_{0}\left(\mu_{k}\right)\right\}}{\operatorname{det}_{M}\left\{\delta_{k}^{j} \mathfrak{a}_{n}^{\prime}\left(\mu_{j} \mid \alpha\right)-K\left(\mu_{j}-\mu_{k}\right)\right\}},
\end{aligned}
$$

where we have introduced the function

$$
\phi(\lambda)=\prod_{j=1}^{M} \frac{\operatorname{sh}\left(\lambda-\mu_{j}\right)}{\operatorname{sh}\left(\lambda-\lambda_{j}\right)} .
$$

Employing now the Cauchy determinant formula written in the form

$$
\prod_{1 \leq j<k \leq M} \frac{\operatorname{sh}\left(\mu_{j}-\mu_{k}\right)}{\operatorname{sh}\left(\lambda_{j}-\lambda_{k}\right)}=\operatorname{det}_{M}\left\{\frac{\operatorname{res} \phi\left(\lambda_{k}\right)}{\operatorname{sh}\left(\lambda_{k}-\mu_{j}\right)}\right\}
$$

and the identity

$$
\phi\left(\mu_{k}-\eta\right) \phi^{-1}\left(\mu_{k}+\eta\right)=-q^{-2 \alpha} \mathfrak{a}_{0}\left(\mu_{k}\right),
$$

which follows from the Bethe ansatz equations for the $\mu_{k}$, we can simplify the right hand side of A.1. We find that

$$
\begin{aligned}
& {\left[\prod_{1 \leq j<k \leq M} \frac{\operatorname{sh}\left(\mu_{j}-\mu_{k}\right)}{\operatorname{sh}\left(\lambda_{j}-\lambda_{k}\right)}\right]} \\
& \times\left[\prod_{j=1}^{M}-\mathrm{e}^{\lambda_{j}-\mu_{j}} q^{-\alpha} \phi^{-1}\left(\mu_{k}-\eta\right)\right] \operatorname{det}_{M}\left\{\mathrm{e}\left(\lambda_{j}-\mu_{k}\right)-\mathrm{e}\left(\mu_{k}-\lambda_{j}\right) \mathfrak{a}_{0}\left(\mu_{k}\right)\right\} \\
& =\operatorname{det}_{M}\left\{\frac{\mathrm{e}^{\lambda_{\ell}-\mu_{j}} \operatorname{res} \phi\left(\lambda_{\ell}\right)}{\operatorname{sh}\left(\mu_{j}-\lambda_{\ell}\right)}\right\} \operatorname{det}_{M}\left\{q^{-\alpha} \phi^{-1}\left(\mu_{k}-\eta\right) \mathrm{e}\left(\lambda_{\ell}-\mu_{k}\right)+q^{\alpha} \phi^{-1}\left(\mu_{k}+\eta\right) \mathrm{e}\left(\mu_{k}-\lambda_{\ell}\right)\right\} \\
& =\operatorname{det}_{M}\left\{\int_{\mathcal{C}} \frac{\mathrm{d} \lambda}{2 \pi \mathrm{i}} \frac{\mathrm{e}^{\lambda-\mu_{j}} \phi(\lambda)}{\operatorname{sh}\left(\mu_{j}-\lambda\right)}\left(q^{-\alpha} \phi^{-1}\left(\mu_{k}-\eta\right) \mathrm{e}\left(\lambda-\mu_{k}\right)+q^{\alpha} \phi^{-1}\left(\mu_{k}+\eta\right) \mathrm{e}\left(\mu_{k}-\lambda\right)\right)\right\} \\
& =\operatorname{det}_{M}\left\{\delta_{k}^{j} \rho_{n}^{-1}\left(\mu_{j} \mid \alpha\right) \mathfrak{a}_{n}^{\prime}\left(\mu_{j} \mid \alpha\right)-\frac{q^{1+\alpha}}{\operatorname{sh}\left(\mu_{j}-\mu_{k}-\eta\right)}+\frac{q^{-1-\alpha}}{\operatorname{sh}\left(\mu_{j}-\mu_{k}+\eta\right)}\right\} .
\end{aligned}
$$

The contour $\mathcal{C}$ in the second equation is chosen in such a way that all Bethe roots $\lambda_{j}$, $j=1, \ldots, M$, are included, but $\mu_{j}, \mu_{k}$ and $\mu_{k} \pm \eta$ are excluded. The integral can be 
calculated, since the integrand is an $i \pi$-periodic function of $\lambda$ which decreases to zero as $\operatorname{Re} \lambda \rightarrow \pm \infty$ *** In the last equation we have inserted the identity

$$
\phi^{\prime}\left(\mu_{j}\right)\left(q^{-\alpha} \phi^{-1}\left(\mu_{j}-\eta\right)-q^{\alpha} \phi^{-1}\left(\mu_{j}+\eta\right)\right)=\rho_{n}^{-1}\left(\mu_{j} \mid \alpha\right) \mathfrak{a}_{n}^{\prime}\left(\mu_{j} \mid \alpha\right)
$$

which follows from Baxter's $T Q$-equation. Comparing (A.1) and A.5 and taking into account the definition 39 of the kernel $\mathcal{K}_{\alpha}$ we obtain

$$
\left[\prod_{j=1}^{M} \frac{q^{-\alpha} d\left(\mu_{j}\right) \mathrm{e}^{\lambda_{j}}}{d\left(\lambda_{j}\right) \mathrm{e}^{\mu_{j}}}\right] \frac{\left\langle\Psi_{n}^{\alpha} \mid \Psi_{0}\right\rangle}{\left\langle\Psi_{n}^{\alpha} \mid \Psi_{n}^{\alpha}\right\rangle}=\left[\prod_{j=1}^{M} \rho_{n}^{-1}\left(\mu_{j} \mid \alpha\right)\right] \frac{\operatorname{det}_{M}\left\{\delta_{k}^{j}-\frac{\rho_{n}\left(\mu_{j} \mid \alpha\right)}{\mathfrak{a}_{n}^{\prime}\left(\mu_{j} \mid \alpha\right)} \mathcal{K}_{-\alpha}\left(\mu_{j}-\mu_{k}\right)\right\}}{\operatorname{det}_{M}\left\{\delta_{k}^{j}-\frac{1}{\mathfrak{a}_{n}^{\prime}\left(\mu_{j} \mid \alpha\right)} \mathcal{K}\left(\mu_{j}-\mu_{k}\right)\right\}} .
$$

A very similar calculation with the roles of $\lambda_{j}$ and $\mu_{k}$ interchanged yields

$$
\left[\prod_{j=1}^{M} \frac{q^{\alpha} d\left(\lambda_{j}\right) \mathrm{e}^{\mu_{j}}}{d\left(\mu_{j}\right) \mathrm{e}^{\lambda_{j}}}\right] \frac{\left\langle\Psi_{0} \mid \Psi_{n}^{\alpha}\right\rangle}{\left\langle\Psi_{0} \mid \Psi_{0}\right\rangle}=\left[\prod_{j=1}^{M} \rho_{n}\left(\lambda_{j} \mid \alpha\right)\right] \frac{\operatorname{det}_{M}\left\{\delta_{k}^{j}-\frac{\rho_{n}^{-1}\left(\lambda_{j} \mid \alpha\right)}{\mathfrak{a}_{0}^{\prime}\left(\lambda_{j}\right)} \mathcal{K}_{\alpha}\left(\lambda_{j}-\lambda_{k}\right)\right\}}{\operatorname{det}_{M}\left\{\delta_{k}^{j}-\frac{1}{\mathfrak{a}_{0}^{\prime}\left(\lambda_{j}\right)} \mathcal{K}\left(\lambda_{j}-\lambda_{k}\right)\right\}} .
$$

Then the equation (38) for the amplitudes follows by multiplying (A.7) and A.8.

\section{Appendix B: Amplitudes of the transversal correlation functions}

In this appendix we derive the expressions 55 and 61$]$ for the form factors $F_{+}(\xi)$ and $F_{-}(\xi)$ defined in 52 .

\section{B.1 The form factor $F_{+}(\xi)$}

Now the excited state $\left|\Psi_{n}^{\alpha}\right\rangle$ is parameterized by $M-1$ Bethe roots still denoted $\mu_{j}$. Again a function $\phi$, now defined by

$$
\phi(\lambda)=\frac{\prod_{j=1}^{M-1} \operatorname{sh}\left(\lambda-\mu_{j}\right)}{\prod_{j=1}^{M} \operatorname{sh}\left(\lambda-\lambda_{j}\right)}
$$

will be a useful tool in our calculation. We further use a degenerate variant of the Cauchy determinant formula obtained by sending the real part of one of the variables ( $\mu_{M}$ in our case) to $-\infty$ and picking up the asymptotically leading term,

$$
\frac{\prod_{1 \leq j<k \leq M-1} \operatorname{sh}\left(\mu_{j}-\mu_{k}\right)}{\prod_{1 \leq j<k \leq M} \operatorname{sh}\left(\lambda_{j}-\lambda_{k}\right)}=\left[\prod_{j=1}^{M} \operatorname{res} \phi\left(\lambda_{j}\right)\right] \operatorname{det}\left(\begin{array}{ccc}
\frac{\mathrm{e}^{\lambda_{1}-\mu_{1}}}{\operatorname{sh}\left(\lambda_{1}-\mu_{1}\right)} & \cdots & \frac{\mathrm{e}^{\lambda_{M}-\mu_{1}}}{\operatorname{sh}\left(\lambda_{M}-\mu_{1}\right)} \\
\vdots & & \vdots \\
\frac{\mathrm{e}^{\lambda_{1}-\mu_{M-1}}}{\operatorname{sh}\left(\lambda_{1}-\mu_{M-1}\right)} & \cdots & \frac{\mathrm{e}^{\lambda_{M}-\mu_{M-1}}}{\operatorname{sh}\left(\lambda_{M}-\mu_{M-1}\right)} \\
1 & \cdots & 1
\end{array}\right)
$$

Inserting the determinant formula (36) and the expression (17) for the eigenvalue into the definition 52 of $F_{-}(\xi)$ and using the above formulae we obtain

$$
F_{+}(\xi)=A \operatorname{det}_{M}\left(\left(\begin{array}{l}
C \\
E
\end{array}\right) \mathbf{u}_{1}, \ldots,\left(\begin{array}{l}
C \\
E
\end{array}\right) \mathbf{u}_{M}\right)
$$

\footnotetext{
${ }^{* *}$ A very similar calculation, inspired by [16], was performed in the appendix of [4].
} 
where

$$
A=\frac{\prod_{j=1}^{M} d\left(\lambda_{j}\right) \mathrm{e}^{\lambda_{j}}}{\prod_{j=1}^{M-1} q^{-\alpha} d\left(\mu_{j}\right) \mathrm{e}^{\mu_{j}}} \frac{1}{\operatorname{det}_{M-1}\left\{\delta_{k}^{j} \mathfrak{a}_{n}^{\prime}\left(\mu_{j} \mid \alpha\right)-K\left(\mu_{j}-\mu_{k}\right)\right\}},
$$

where

$$
\begin{aligned}
C_{\ell}^{j} & =\frac{1}{\operatorname{sh}\left(\lambda_{\ell}-\mu_{j}\right)}, \quad j=1, \ldots, M-1 ; \ell=1, \ldots, M, \\
E_{\ell}^{1} & =\mathrm{e}^{-\lambda_{\ell}}, \quad \ell=1, \ldots, M,
\end{aligned}
$$

and where

$$
\begin{array}{r}
\left(\mathbf{u}_{k}\right)^{\ell}=-\operatorname{res} \phi\left(\lambda_{\ell}\right)\left\{q^{-\alpha} \phi^{-1}\left(\mu_{k}-\eta\right) \mathrm{e}\left(\lambda_{\ell}-\mu_{k}\right)+q^{\alpha} \phi^{-1}\left(\mu_{k}+\eta\right) \mathrm{e}\left(\mu_{k}-\lambda_{\ell}\right)\right\}, \\
k=1, \ldots, M-1 ; \ell=1, \ldots, M, \\
\left(\mathbf{u}_{M}\right)^{\ell}=-\frac{\phi^{-1}(\xi)}{1+\mathfrak{a}_{0}(\xi)} \operatorname{res} \phi\left(\lambda_{\ell}\right)\left\{\mathrm{e}\left(\lambda_{\ell}-\xi\right)-\mathrm{e}\left(\xi-\lambda_{\ell}\right) \mathfrak{a}_{0}(\xi)\right\}, \quad \ell=1, \ldots, M .
\end{array}
$$

The determinant on the right hand side of (B.3) can be simplified in a similar way as shown in $\mathrm{A} .5$ for one of the form factors of the generating function. The four cases $C \mathbf{u}_{k}, k=1, \ldots, M-1, C \mathbf{u}_{M}, E \mathbf{u}_{k}, k=1, \ldots, M-1$, and $E \mathbf{u}_{M}$ have to be treated separately. We obtain

$$
\begin{aligned}
\left(C \mathbf{u}_{k}\right)^{j} & =\delta_{k}^{j} \mathfrak{a}_{n}^{\prime}\left(\mu_{j} \mid \alpha\right) \rho_{n}^{-1}\left(\mu_{j} \mid \alpha\right)-\frac{q^{\alpha}}{\operatorname{sh}\left(\mu_{j}-\mu_{k}-\eta\right)}+\frac{q^{-\alpha}}{\operatorname{sh}\left(\mu_{j}-\mu_{k}+\eta\right)} \\
E \mathbf{u}_{k} & =\left(q^{\alpha-1}-q^{1-\alpha}\right) \mathrm{e}^{-\mu_{k}} \\
\left(C \mathbf{u}_{M}\right)^{j} & =\frac{1}{\operatorname{sh}\left(\xi-\mu_{j}\right)}-\frac{q^{-\alpha} \rho_{n}(\xi \mid \alpha)}{1+\mathfrak{a}_{n}(\xi \mid \alpha)} \frac{1}{\operatorname{sh}\left(\xi-\mu_{j}-\eta\right)}-\frac{q^{\alpha} \rho_{n}(\xi \mid \alpha)}{1+\overline{\mathfrak{a}}_{n}(\xi \mid \alpha)} \frac{1}{\operatorname{sh}\left(\xi-\mu_{j}+\eta\right)} \\
E \mathbf{u}_{M} & =\mathrm{e}^{-\xi}\left(1-\frac{q^{1-\alpha} \rho_{n}(\xi \mid \alpha)}{1+\mathfrak{a}_{n}(\xi \mid \alpha)}-\frac{q^{\alpha-1} \rho_{n}(\xi \mid \alpha)}{1+\overline{\mathfrak{a}}_{n}(\xi \mid \alpha)}\right)
\end{aligned}
$$

where $\overline{\mathfrak{a}}_{n}=1 / \mathfrak{a}_{n}$ by definition.

If we directly continue with these matrices we end up with Fredholm determinants with kernel $\mathcal{K}$. Having in mind the more involved case of the form factor $F_{-}(\xi)$ we prefer to switch at this point to the kernel $K$. For this purpose we subtract $\mathrm{e}^{\mu_{j}}$ times $(\mathrm{B} .7 \mathrm{~b})$ from $(\mathrm{B} .7 \mathrm{a})$ and $\mathrm{e}^{\mu_{j}}$ times $(\mathrm{B} .7 \mathrm{~d})$ from $(\mathrm{B} .7 \mathrm{c})$ which does not change the determinant in $(\mathrm{B} .3)$. Then

$$
\begin{aligned}
\left(C \mathbf{u}_{k}\right)^{j}-\mathrm{e}^{\mu_{j}} E \mathbf{u}_{k}= & \delta_{k}^{j} \mathfrak{a}_{n}^{\prime}\left(\mu_{j} \mid \alpha\right) \rho_{n}^{-1}\left(\mu_{j} \mid \alpha\right)-\mathrm{e}^{\mu_{j}-\mu_{k}} K_{1-\alpha}\left(\mu_{j}-\mu_{k}\right) \\
= & \mathrm{e}^{\mu_{j}-\mu_{k}}\left(\mathbf{v}_{k}\right)^{j}, \\
\left(C \mathbf{u}_{M}\right)^{j}-\mathrm{e}^{\mu_{j}} E \mathbf{u}_{M}= & \mathrm{e}^{\mu_{j}-\xi}\left[\operatorname{cth}\left(\xi-\mu_{j}\right)-\frac{q^{1-\alpha} \rho_{n}(\xi \mid \alpha)}{1+\mathfrak{a}_{n}(\xi \mid \alpha)} \operatorname{cth}\left(\xi-\mu_{j}-\eta\right)\right. \\
& \left.\quad-\frac{q^{\alpha-1} \rho_{n}(\xi \mid \alpha)}{1+\overline{\mathfrak{a}}_{n}(\xi \mid \alpha)} \operatorname{cth}\left(\xi-\mu_{j}+\eta\right)\right] \\
= & \mathrm{e}^{\mu_{j}-\xi}\left(\mathbf{v}_{M}\right)^{j},
\end{aligned}
$$


where $j=1, \ldots, M-1$. These equations define an $(M-1) \times(M-1)$ matrix $V$ with matrix elements $V_{k}^{j}=\left(\mathbf{v}_{k}\right)^{j}$ and an $(M-1)$-component column vector $\mathbf{v}_{M}$.

Using $(\overline{B .8})$ in $(\mathrm{B} .3)$ and expanding the determinant with respect to its last row we see that

$$
F_{+}(\xi)=A \mathrm{e}^{-\xi} \operatorname{det}(V)\left\{E \mathbf{u}_{M-1} \mathrm{e}^{\xi}-\left(E \mathbf{u}_{1} \mathrm{e}^{\mu_{1}}, \ldots, E \mathbf{u}_{M-1} \mathrm{e}^{\mu_{M-1}}\right) V^{-1} \mathbf{v}_{M}\right\}
$$

Here we have assumed that $V$ is invertible, i.e. that

$$
\operatorname{det}_{M-1}(V)=\left[\prod_{j=1}^{M-1} \frac{\mathfrak{a}_{n}^{\prime}\left(\mu_{j} \mid \alpha\right)}{\rho_{n}\left(\mu_{j} \mid \alpha\right)}\right] \operatorname{det}_{M-1}\left\{\delta_{k}^{j}-\frac{\rho_{n}\left(\mu_{j} \mid \alpha\right)}{\mathfrak{a}_{n}^{\prime}\left(\mu_{j} \mid \alpha\right)} K_{1-\alpha}\left(\mu_{j}-\mu_{k}\right)\right\}
$$

is non-zero. We do not have a general proof of this assumption, but we checked it with several examples for small $M$.

It remains to calculate the terms in curly brackets on the right hand side of (B.9). Setting $\mathbf{x}=V^{-1} \mathbf{v}_{M}$ we find that the coordinates $x^{j}$ of this vector satisfy the set of linear equations

$$
\begin{aligned}
\mathfrak{a}_{n}^{\prime}\left(\mu_{j} \mid \alpha\right) \rho_{n}^{-1}\left(\mu_{j} \mid \alpha\right) x^{j} & =-\operatorname{cth}\left(\mu_{j}-\xi\right)+\frac{q^{1-\alpha} \rho_{n}(\xi \mid \alpha)}{1+\mathfrak{a}_{n}(\xi \mid \alpha)} \operatorname{cth}\left(\mu_{j}-\xi+\eta\right) \\
+ & \frac{q^{\alpha-1} \rho_{n}(\xi \mid \alpha)}{1+\overline{\mathfrak{a}}_{n}(\xi \mid \alpha)} \operatorname{cth}\left(\mu_{j}-\xi-\eta\right)+\sum_{k=1}^{M-1} K_{1-\alpha}\left(\mu_{j}-\mu_{k}\right) x^{k}
\end{aligned}
$$

This suggests to define a function

$$
\begin{aligned}
G_{+}(\lambda, \xi)=-\operatorname{cth}(\lambda-\xi) & +\frac{q^{1-\alpha} \rho_{n}(\xi \mid \alpha)}{1+\mathfrak{a}_{n}(\xi \mid \alpha)} \operatorname{cth}(\lambda-\xi+\eta) \\
& +\frac{q^{\alpha-1} \rho_{n}(\xi \mid \alpha)}{1+\overline{\mathfrak{a}}_{n}(\xi \mid \alpha)} \operatorname{cth}(\lambda-\xi-\eta)+\sum_{k=1}^{M-1} K_{1-\alpha}\left(\lambda-\mu_{k}\right) x^{k}
\end{aligned}
$$

which has the following properties:

$$
G_{+}\left(\mu_{k}, \xi\right)=\mathfrak{a}_{n}^{\prime}\left(\mu_{k} \mid \alpha\right) \rho_{n}^{-1}\left(\mu_{k} \mid \alpha\right) x^{k},
$$

and $G_{+}(\lambda, \xi)$ as a function of $\lambda$ is meromorphic inside the contour $\mathcal{C}_{n}$ with a single simple pole at $\lambda=\xi$ with residue -1 . It follows that $G_{+}$satisfies the linear integral equation

$$
\begin{aligned}
G_{+}(\lambda, \xi) & =-\operatorname{cth}(\lambda-\xi) \\
& +q^{\alpha-1} \rho_{n}(\xi \mid \alpha) \operatorname{cth}(\lambda-\xi-\eta)+\int_{\mathcal{C}_{n}} \mathrm{~d} m_{+}^{\alpha}(\mu) K_{1-\alpha}(\lambda-\mu) G_{+}(\mu, \xi),
\end{aligned}
$$

where

$$
\mathrm{d} m_{+}^{\alpha}(\lambda)=\frac{\mathrm{d} \lambda \rho_{n}(\lambda \mid \alpha)}{2 \pi \mathrm{i}\left(1+\mathfrak{a}_{n}(\lambda \mid \alpha)\right)}
$$


Finally

$$
\begin{aligned}
E \mathbf{u}_{M} \mathrm{e}^{\xi} & -\left(E \mathbf{u}_{1} \mathrm{e}^{\mu_{1}}, \ldots, E \mathbf{u}_{M-1} \mathrm{e}^{\mu_{M-1}}\right) V^{-1} \mathbf{v}_{M}=E \mathbf{u}_{M} \mathrm{e}^{\xi}-\sum_{j=1}^{M-1} E \mathbf{u}_{j} \mathrm{e}^{\mu_{j}} x^{j} \\
& =1-q^{\alpha-1} \rho_{n}(\xi \mid \alpha)-\left(q^{\alpha-1}-q^{1-\alpha}\right) \int_{\mathcal{C}_{n}} \mathrm{~d} m_{+}^{\alpha}(\lambda) G_{+}(\lambda, \xi) \\
& =\lim _{\operatorname{Re} \lambda \rightarrow-\infty} G_{+}(\lambda, \xi)=G_{+}^{-}(\xi) .
\end{aligned}
$$

Here we have used $(\mathrm{B} .13)$ in the second equation. Inserting $(\mathrm{B} .4),(\mathrm{B} .10)$ and $(\mathrm{B} .16)$ into (B.9) we arrive at equation (55) of the main text.

\section{B.2 The form factor $F_{-}(\xi)$}

Here we can proceed as in the previous section by using in $(53)$ the second equation (36) with $\mathfrak{a}_{0}$ on the right hand side. We obtain a formula similar to (55), but with a different determinant in the denominator. Multiplying this expression for $F_{-}(\xi)$ with (55) we obtain equation (58) for the amplitude $A_{n}^{-+}(\xi)$. In order to obtain the simpler and more symmetric formula 64 of the main text, however, we have to perform a more cumbersome calculation which we shall sketch below.

Instead of using (36) directly in 53 ) we first of all move $B(\xi)$ through the product of $C$ operators in $\left\langle\Psi_{0}\right|$ to the left, using the well-known Yang-Baxter algebra relations (cf. e.g. [15, 36]). This produces a double sum $F_{-}(\xi)=\sum_{\substack{\ell, m \\ \ell \neq m}}^{M+1} t_{\ell, m}$, where

$$
\begin{aligned}
t_{\ell, m}=d\left(\lambda_{\ell}\right) a\left(\lambda_{m}\right) c\left(\lambda_{\ell}, \xi\right) c\left(\xi, \lambda_{m}\right) & \\
& {\left[\prod_{\substack{k=1 \\
k \neq \ell}}^{M+1} \frac{1}{b\left(\lambda_{\ell}, \lambda_{k}\right)}\right]\left[\prod_{\substack{k=1 \\
k \neq \ell, m}}^{M+1} \frac{1}{b\left(\lambda_{k}, \lambda_{m}\right)}\right] \frac{\left\langle 0\left|\prod_{\substack{k=1 \\
k \neq \ell, m}}^{M+1} C\left(\lambda_{k}\right)\right| \Psi_{n}^{\alpha}\right\rangle}{\Lambda_{n}(\xi \mid \alpha)\left\langle\Psi_{0} \mid \Psi_{0}\right\rangle} }
\end{aligned}
$$

and $\lambda_{M+1}=\xi$ in the various products above. Here we insert the scalar product formula (36) on the right hand side. The result can be written in the following form,

$$
\begin{aligned}
t_{\ell, m}=\frac{B \rho_{n}^{-1}(\xi \mid \alpha)}{1+\mathfrak{a}_{0}(\xi)} \operatorname{det}_{M}(D) \operatorname{det}_{M-1}\left\{\phi\left(\lambda_{\ell_{k}}-\eta\right)\left[\mathrm{e}\left(\mu_{j}-\lambda_{\ell_{k}}\right)-\mathrm{e}\left(\lambda_{\ell_{k}}-\mu_{j}\right) \mathfrak{a}_{n}\left(\lambda_{\ell_{k}} \mid \alpha\right)\right]\right\} \\
\times \frac{\left(-\mathfrak{a}_{0}\left(\lambda_{\ell}\right)\right) \operatorname{sign}(m-\ell)(-1)^{\ell-m}}{\operatorname{sh}\left(\lambda_{\ell}-\lambda_{m}+\eta\right)},
\end{aligned}
$$

where $\ell_{k} \in\{1, \ldots, M+1\} \backslash\{\ell, m\}, k=1, \ldots, M-1, \ell_{1}<\ell_{2}<\cdots<\ell_{M-1}$, and

$$
\begin{gathered}
B=\frac{\prod_{j=1}^{M-1} q^{-\alpha} d\left(\mu_{j}\right) \mathrm{e}^{\mu_{j}}}{\prod_{j=1}^{M} d\left(\lambda_{j}\right) \mathrm{e}^{\lambda_{j}}} \frac{1}{\operatorname{det}_{M}\left\{\delta_{k}^{j} \mathfrak{a}_{0}^{\prime}\left(\lambda_{j}\right)-K\left(\lambda_{j}-\lambda_{k}\right)\right\}}, \\
D=\left(\begin{array}{cccc}
\frac{q^{\alpha} \operatorname{res} \phi^{-1}\left(\mu_{1}\right)}{\operatorname{sh}\left(\lambda_{1}-\mu_{1}\right)} & \ldots & \frac{q^{\alpha} \operatorname{res} \phi^{-1}\left(\mu_{M-1}\right)}{\operatorname{sh}\left(\lambda_{1}-\mu_{M-1}\right)} & \mathrm{e}^{\lambda_{1}} \\
\vdots & & \vdots & \vdots \\
\frac{q^{\alpha} \operatorname{res} \phi^{-1}\left(\mu_{1}\right)}{\operatorname{sh}\left(\lambda_{M}-\mu_{1}\right)} & \ldots & \frac{q^{\alpha} \operatorname{res} \phi^{-1}\left(\mu_{M-1}\right)}{\operatorname{sh}\left(\lambda_{M}-\mu_{M-1}\right)} & \mathrm{e}^{\lambda_{M}}
\end{array}\right)
\end{gathered}
$$


The function $\phi$ was defined in equation $(\overline{B .1})$ above. At this point we see already that the prefactor in $B$ which would be somewhat inconvenient in the Trotter limit will cancel the corresponding prefactor in A, equation (B.4). What remains to be done is to sum up the $t_{\ell, m}$ into a single determinant. We shall divide this task into several steps.

Let us define

$$
\begin{aligned}
\left(\mathbf{a}_{k}\right)^{j} & =\phi\left(\lambda_{k}-\eta\right)\left(\mathrm{e}\left(\mu_{j}-\lambda_{k}\right)-\mathrm{e}\left(\lambda_{k}-\mu_{j}\right) \mathfrak{a}_{n}\left(\lambda_{k} \mid \alpha\right)\right), \\
s_{k j} & =\frac{1}{\operatorname{sh}\left(\lambda_{j}-\lambda_{k}+\eta\right)}
\end{aligned}
$$

where $j=1, \ldots, M-1$ in $(\mathrm{B} .20 \mathrm{a}$ and where the remaining indices run from 1 to $M+1$. Then

$$
\begin{array}{r}
\sum_{\ell=1}^{M} t_{\ell, M+1}=\frac{B \rho_{n}^{-1}(\xi \mid \alpha)}{1+\mathfrak{a}_{0}(\xi)} \operatorname{det}_{M}(D) \sum_{\ell=1}^{M}(-1)^{M+\ell-1} s_{M+1, \ell} \operatorname{det}_{M-1}\left(\mathbf{a}_{1}, \ldots, \mathbf{a}_{\ell-1}, \mathbf{a}_{\ell+1}, \ldots, \mathbf{a}_{M}\right) \\
=-\frac{B \rho_{n}^{-1}(\xi \mid \alpha)}{1+\mathfrak{a}_{0}(\xi)} \operatorname{det}\left(D\left(\begin{array}{c}
\mathbf{a}_{1} \\
s_{M+1,1}
\end{array}\right), \ldots, D\left(\begin{array}{c}
\mathbf{a}_{M} \\
s_{M+1, M}
\end{array}\right)\right) . \quad \text { (B.21) }
\end{array}
$$

We denote the matrix in the determinant on the right hand side of this equation by $X$. The entries of this matrix can be simplified the same way as in the previous section, and

$$
X_{k}^{j}=\delta_{k}^{j} \rho_{n}\left(\lambda_{j} \mid \alpha\right) \mathfrak{a}_{0}^{\prime}\left(\lambda_{j}\right)-\frac{q^{-\alpha}}{\operatorname{sh}\left(\lambda_{j}-\lambda_{k}-\eta\right)}+\frac{q^{\alpha}}{\operatorname{sh}\left(\lambda_{j}-\lambda_{k}+\eta\right)}+\mathrm{e}^{\lambda_{j}} s_{M+1, k}
$$

This expression looks appealing as it is a sum of a matrix and a one-dimensional projector. One might be tempted to pull the matrix $X_{k}^{j}-\mathrm{e}^{\lambda_{j}} s_{M+1, k}$ out of the determinant. This is impossible, however, since the matrix is not invertible. The latter fact can be easily seen by replacing $s_{M+1, k}$ on the right hand side of (B.21) by zero. A left eigenvector with zero eigenvalue has the components $\operatorname{res} \phi\left(\lambda_{j}\right)$.

In order to resolve the problem we introduce the kernel $K$ at this point and write

$$
\begin{aligned}
\operatorname{det}(X) & =\operatorname{det}_{M}\left\{\delta_{k}^{j} \rho_{n}\left(\lambda_{j} \mid \alpha\right) \mathfrak{a}_{0}^{\prime}\left(\lambda_{j}\right)-K_{1+\alpha}\left(\lambda_{j}-\lambda_{k}\right)+\widetilde{s}_{M+1, k}\right\} \\
\widetilde{s}_{j, k} & =\mathrm{e}^{\lambda_{k}} s_{j, k}+q^{-1-\alpha}-q^{1+\alpha}
\end{aligned}
$$

We further define

$$
\widehat{K}_{k}^{j}=\frac{K_{1+\alpha}\left(\lambda_{j}-\lambda_{k}\right)}{\rho_{n}\left(\lambda_{j} \mid \alpha\right) \mathfrak{a}_{0}^{\prime}\left(\lambda_{j}\right)}, \quad \mathbf{v}^{j}=\frac{1}{\rho_{n}\left(\lambda_{j} \mid \alpha\right) \mathfrak{a}_{0}^{\prime}\left(\lambda_{j}\right)}
$$

and assume henceforth that $1-\widehat{K}$ is invertible. This seems to be true for general $M$. It is easy to prove for $M=1$, and for larger $M$ we verified it numerically for a number of examples. Setting

$$
\mathbf{w}=(1-\widehat{K})^{-1} \mathbf{v}
$$

we conclude that

$$
\underset{M}{\operatorname{det}}(X)=\left[\prod_{j=1}^{M} \frac{\mathfrak{a}_{0}^{\prime}\left(\lambda_{j}\right)}{\rho_{n}^{-1}\left(\lambda_{j} \mid \alpha\right)}\right] \operatorname{det}(1-\widehat{K})\left[1+\sum_{j=1}^{M} \widetilde{s}_{M+1, j} \mathbf{w}^{j}\right] .
$$


As before we convert $(\mathrm{B} .25)$ into an integral equation. It is equivalent to the linear equation

$$
\mathbf{w}^{j} \rho_{n}\left(\lambda_{j} \mid \alpha\right) \mathfrak{a}_{0}^{\prime}\left(\lambda_{j}\right)=1+\sum_{k=1}^{M} K_{1+\alpha}\left(\lambda_{j}-\lambda_{k}\right) \mathbf{w}^{k}
$$

Defining

$$
\sigma(\lambda)=1+\sum_{k=1}^{M} K_{1+\alpha}\left(\lambda-\lambda_{k}\right) \mathbf{w}^{k}
$$

we conclude that

$$
\mathbf{w}^{j}=\frac{\sigma\left(\lambda_{j}\right)}{\rho_{n}\left(\lambda_{j} \mid \alpha\right) \mathfrak{a}_{0}^{\prime}\left(\lambda_{j}\right)}
$$

and

$$
\sigma(\lambda)=1+\int_{\mathfrak{C}_{n}} \mathrm{~d} m_{-}^{\alpha}(\mu) K_{1+\alpha}(\lambda-\mu) \sigma(\mu) .
$$

The function $\sigma(\lambda)$ is a generalized dressed charge function of the type introduced in [5]. It allows us to write

$$
1+\sum_{j=1}^{M} \widetilde{s}_{M+1, j} \mathbf{w}^{j}=\sigma_{\infty}-\int_{\mathfrak{C}_{n}} \mathrm{~d} \bar{m}_{-}^{\alpha}(\lambda) \frac{\mathrm{e}^{\lambda} \sigma(\lambda)}{\operatorname{sh}(\lambda-\xi+\eta)},
$$

where

$$
\mathrm{d} \bar{m}_{-}^{\alpha}(\lambda)=\frac{\mathrm{d} \lambda \rho_{n}^{-1}(\lambda \mid \alpha)}{2 \pi \mathrm{i}\left(1+\overline{\mathfrak{a}}_{0}(\lambda)\right)}
$$

We finally argue that

$$
\sigma_{\infty}=\lim _{\operatorname{Re} \lambda \rightarrow+\infty} \sigma(\lambda)=0 .
$$

This can be seen as follows. We could have introduced a parameter, $\gamma$ say, into the last column of the matrix $D$ above and at the same time could have multiplied the whole sum (B.21) we are considering by $1 / \gamma$. This trivial modification cannot change our result, but we would have a term $\sigma_{\infty} / \gamma$ instead of $\sigma_{\infty}$ in the final formula, whence (B.33) must hold. Inserting (B.26), (B.31) and (B.33) into (B.21) we obtain

$$
\sum_{\ell=1}^{M} t_{\ell, M+1}=\frac{B \rho_{n}^{-1}(\xi \mid \alpha)}{1+\mathfrak{a}_{0}(\xi)}\left[\prod_{j=1}^{M} \frac{\mathfrak{a}_{0}^{\prime}\left(\lambda_{j}\right)}{\rho_{n}^{-1}\left(\lambda_{j} \mid \alpha\right)}\right] \operatorname{det}_{M}(1-\widehat{K}) \int_{\mathcal{C}_{n}} \mathrm{~d} \bar{m}_{-}^{\alpha}(\lambda) \frac{\mathrm{e}^{\lambda} \sigma(\lambda)}{\operatorname{sh}(\lambda-\xi+\eta)} .
$$

In a similar way

$$
\sum_{m=1}^{M} t_{M+1, m}=-\frac{B \rho_{n}^{-1}(\xi \mid \alpha)}{1+\overline{\mathfrak{a}}_{0}(\xi)}\left[\prod_{j=1}^{M} \frac{\mathfrak{a}_{0}^{\prime}\left(\lambda_{j}\right)}{\rho_{n}^{-1}\left(\lambda_{j} \mid \alpha\right)}\right] \operatorname{det}(1-\widehat{K}) \int_{\mathcal{C}_{n}} \mathrm{~d} m_{-}^{\alpha}(\lambda) \frac{\mathrm{e}^{\lambda} \sigma(\lambda)}{\operatorname{sh}(\xi-\lambda+\eta)} .
$$

To prepare for the calculation of the remaining double sum with $\ell, m<M+1$ we introduce the $M \times M$ matrix

$$
U_{m}=\left(\begin{array}{ccccccc}
\mathbf{a}_{1} & \ldots & \mathbf{a}_{m-1} & \mathbf{a}_{M+1} & \mathbf{a}_{m+1} & \ldots & \mathbf{a}_{M} \\
s_{m, 1} & \ldots & s_{m, m-1} & s_{m, m} & s_{m, m+1} & \ldots & s_{m, M}
\end{array}\right)
$$


Then

$$
\begin{aligned}
\sum_{\substack{\ell=1 \\
\ell \neq m}}^{M} t_{\ell, m}=\frac{B \rho_{n}^{-1}(\xi \mid \alpha)}{1+\mathfrak{a}_{0}(\xi)} \operatorname{det}(D) & \\
& \times\left\{\operatorname{det}_{M}\left(U_{m}\right)-(-1)^{M-m} s_{m, m} \operatorname{det}_{M-1}\left(\mathbf{a}_{1}, \ldots, \mathbf{a}_{m-1}, \mathbf{a}_{m+1}, \ldots, \mathbf{a}_{M}\right)\right\}
\end{aligned}
$$

which still has to be summed over $m$.

For the second term in curly brackets we can proceed as above and obtain

$$
\begin{aligned}
\frac{B \rho_{n}^{-1}(\xi \mid \alpha)}{1+\mathfrak{a}_{0}(\xi)} & \operatorname{det}(D) \sum_{m=1}^{M}(-1)^{M-m+1} s_{m, m} \operatorname{det}_{M-1}\left(\mathbf{a}_{1}, \ldots, \mathbf{a}_{m-1}, \mathbf{a}_{m+1}, \ldots, \mathbf{a}_{M}\right) \\
= & -\frac{B \rho_{n}^{-1}(\xi \mid \alpha)}{1+\mathfrak{a}_{0}(\xi)}\left[\prod_{j=1}^{M} \frac{\mathfrak{a}_{0}^{\prime}\left(\lambda_{j}\right)}{\rho_{n}^{-1}\left(\lambda_{j} \mid \alpha\right)}\right] \operatorname{det}(1-\widehat{K}) \int_{\mathcal{C}_{n}} \mathrm{~d} m_{-}^{\alpha}(\lambda) \frac{\mathrm{e}^{\lambda} \sigma(\lambda)}{\operatorname{sh}(\eta)}
\end{aligned}
$$

For the first term we first simplify $D U_{m}$ and perform the summation afterwards. Thus,

$$
\begin{aligned}
\left(D U_{m}\right)_{k}^{j} & =\delta_{k}^{j} \rho_{n}\left(\lambda_{j} \mid \alpha\right) \mathfrak{a}_{0}^{\prime}\left(\lambda_{j}\right)-\mathrm{e}^{\lambda_{j}-\lambda_{k}}\left(K_{1+\alpha}\left(\lambda_{j}-\lambda_{k}\right)-\widetilde{s}_{m, k}\right), \quad \text { for } k \neq m, \\
\left(D U_{m}\right)_{m}^{j} & =\mathrm{e}^{\lambda_{j}-\xi}\left(\rho_{n}\left(\lambda_{j} \mid \alpha\right) \mathfrak{a}_{0}^{\prime}\left(\lambda_{j}\right) \mathbf{y}^{j}+\widetilde{s}\right)
\end{aligned}
$$

where

$$
\begin{aligned}
& \begin{array}{l}
\rho_{n}\left(\lambda_{j} \mid \alpha\right) \mathfrak{a}_{0}^{\prime}\left(\lambda_{j}\right) \mathbf{y}^{j}=- \\
\quad \rho_{n}(\xi \mid \alpha)\left(1+\mathfrak{a}_{0}(\xi)\right) \operatorname{cth}\left(\lambda_{j}-\xi\right) \\
\quad+q^{\alpha+1} \operatorname{cth}\left(\lambda_{j}-\xi+\eta\right)+\mathfrak{a}_{0}(\xi) q^{-\alpha-1} \operatorname{cth}\left(\lambda_{j}-\xi-\eta\right),
\end{array} \\
& \widetilde{s}=\rho_{n}(\xi \mid \alpha)\left(1+\mathfrak{a}_{0}(\xi)\right)-q^{\alpha+1}-\mathfrak{a}_{0}(\xi) q^{-\alpha-1}+\mathrm{e}^{\xi} s_{m, m},
\end{aligned}
$$

and hence

$$
\begin{aligned}
\operatorname{det}_{M}\left(D U_{m}\right)=\mathrm{e}^{\lambda_{m}-\xi}\left[\prod_{j=1}^{M} \frac{\mathfrak{a}_{0}^{\prime}\left(\lambda_{j}\right)}{\rho_{n}^{-1}\left(\lambda_{j} \mid \alpha\right)}\right] & \operatorname{det}(1-\widehat{K}) \\
& \times\left[\mathbf{z}^{m}+\widetilde{s} \mathbf{w}^{m}+\sum_{\ell=1}^{M} \widetilde{s}_{m, \ell} \operatorname{det}\left(\begin{array}{cc}
\mathbf{w}^{\ell} & \mathbf{z}^{\ell} \\
\mathbf{w}_{m} & \mathbf{z}^{m}
\end{array}\right)\right],
\end{aligned}
$$

where we defined

$$
\mathbf{z}=(1-\widehat{K})^{-1} \mathbf{y} .
$$

Once again we convert (B.42) into an integral equation. Setting

$$
\begin{aligned}
G_{-}(\lambda, \xi) & =-\operatorname{cth}(\lambda-\xi)+\frac{q^{\alpha+1} \rho_{n}^{-1}(\xi \mid \alpha)}{1+\mathfrak{a}_{0}(\xi)} \operatorname{cth}(\lambda-\xi+\eta) \\
& +\frac{q^{-\alpha-1} \rho_{n}^{-1}(\xi \mid \alpha)}{1+\overline{\mathfrak{a}}_{0}(\xi)} \operatorname{cth}(\lambda-\xi-\eta)+\sum_{k=1}^{M} K_{1+\alpha}\left(\lambda-\lambda_{k}\right) \frac{\rho_{n}^{-1}(\xi \mid \alpha) \mathbf{z}^{k}}{1+\mathfrak{a}_{0}(\xi)}
\end{aligned}
$$

implies that

$$
\mathbf{z}^{j}=\frac{\rho_{n}(\xi \mid \alpha)\left(1+\mathfrak{a}_{0}(\xi)\right) G_{-}\left(\lambda_{j}, \xi\right)}{\rho_{n}\left(\lambda_{j} \mid \alpha\right) \mathfrak{a}_{0}^{\prime}\left(\lambda_{j}\right)}
$$


and

$$
\begin{aligned}
& G_{-}(\lambda, \xi)=-\operatorname{cth}(\lambda-\xi) \\
& \quad+q^{-\alpha-1} \rho_{n}^{-1}(\xi \mid \alpha) \operatorname{cth}(\lambda-\xi-\eta)+\int_{\mathcal{C}_{n}} \mathrm{~d} m_{-}^{\alpha}(\mu) K_{1+\alpha}(\lambda-\mu) G_{-}(\mu, \xi)
\end{aligned}
$$

Using (B.29) and B.44) we can calculate the sum on the right hand side of B.41) and can convert it into an integral,

$$
\begin{aligned}
\frac{\rho_{n}^{-1}(\xi \mid \alpha)}{1+\mathfrak{a}_{0}(\xi)} \sum_{m=1}^{M} \mathrm{e}^{\lambda_{m}}\left[\mathbf{z}^{m}+\widetilde{s} \mathbf{w}^{m}+\sum_{\ell=1}^{M} \widetilde{s_{m}, \ell} \operatorname{det}\left(\begin{array}{cc}
\mathbf{w}^{\ell} & \mathbf{z}^{\ell} \\
\mathbf{w}_{m} & \mathbf{z}^{m}
\end{array}\right)\right] \\
\quad=\int_{\mathfrak{C}_{n}} \mathrm{~d} m_{-}^{\alpha}(\lambda) \mathrm{e}^{\lambda} \int_{\mathcal{C}_{n}} \mathrm{~d} \bar{m}_{-}^{\alpha}(\mu) \mathrm{e}^{\mu} \frac{G_{-}(\lambda, \xi) \sigma(\mu)-G_{-}(\mu, \xi) \sigma(\lambda)}{\operatorname{sh}(\lambda-\mu-\eta)} \\
+\frac{\mathrm{e}^{\xi} \rho_{n}^{-1}(\xi \mid \alpha)}{1+\mathfrak{a}_{0}(\xi)} \int_{\mathcal{C}_{n}} \mathrm{~d} m_{-}^{\alpha}(\lambda) \frac{\mathrm{e}^{\lambda} \sigma(\lambda)}{\operatorname{sh}(\eta)}+\frac{\mathrm{e}^{\xi} \rho_{n}^{-1}(\xi \mid \alpha)}{1+\overline{\mathfrak{a}}_{0}(\xi)} \int_{\mathcal{C}_{n}} \mathrm{~d} m_{-}^{\alpha}(\lambda) \frac{\mathrm{e}^{\lambda} \sigma(\lambda)}{\operatorname{sh}(\xi-\lambda+\eta)} \\
-\frac{\mathrm{e}^{\xi} \rho_{n}^{-1}(\xi \mid \alpha)}{1+\mathfrak{a}_{0}(\xi)} \int_{\mathcal{C}_{n}} \mathrm{~d} \bar{m}_{-}^{\alpha}(\lambda) \frac{\mathrm{e}^{\lambda} \sigma(\lambda)}{\operatorname{sh}(\lambda-\xi+\eta)}-G_{-}^{+}(\xi) \int_{\mathcal{C}_{n}} \mathrm{~d} \bar{m}_{-}^{\alpha}(\lambda) \mathrm{e}^{\lambda} \sigma(\lambda),
\end{aligned}
$$

where by definition

$$
G_{-}^{+}(\xi)=\lim _{\operatorname{Re} \lambda \rightarrow+\infty} G_{-}(\lambda, \xi) .
$$

Notice that in (B.46) we have used the measure $\mathrm{d} \bar{m}_{-}^{\alpha}$ when rewriting the finite sums as integrals and that we employed (B.33) to simplify the right hand side of $(\mathrm{B} .46)$. Using a very similar argument as in the derivation of $(B .33)$ we find that

$$
G_{-}^{+}(\xi) \int_{\mathcal{C}_{n}} \mathrm{~d} \bar{m}_{-}^{\alpha}(\lambda) \mathrm{e}^{\lambda} \sigma(\lambda)=0
$$

With this we can summarize what we have obtained so far. Combining (B.34), (B.35), (B.37), (B.38), (B.41), B.46) and (B.48) we conclude that

$$
\begin{gathered}
F_{-}(\xi)=\frac{\mathrm{e}^{-\xi} \prod_{j=1}^{M-1} q^{-\alpha} d\left(\mu_{j}\right) \mathrm{e}^{\mu_{j}}}{\prod_{j=1}^{M} d\left(\lambda_{j}\right) \mathrm{e}^{\lambda_{j}} \rho_{n}^{-1}\left(\lambda_{j} \mid \alpha\right)} \frac{\operatorname{det}_{M}\left\{\delta_{k}^{j}-\frac{\rho_{n}^{-1}\left(\lambda_{j} \mid \alpha\right)}{\mathfrak{a}_{0}^{\prime}\left(\lambda_{j} \mid \alpha\right)} K_{1+\alpha}\left(\lambda_{j}-\lambda_{k}\right)\right\}}{\operatorname{det}_{M}\left\{\delta_{k}^{j}-\frac{1}{\mathfrak{a}_{0}^{\prime}\left(\lambda_{j} \mid \alpha\right)} K\left(\lambda_{j}-\lambda_{k}\right)\right\}} \\
\quad \times \int_{\mathfrak{C}_{n}} \mathrm{~d} m_{-}^{\alpha}(\lambda) \mathrm{e}^{\lambda} \int_{\mathfrak{C}_{n}} \mathrm{~d}_{-}^{\alpha}(\mu) \mathrm{e}^{\mu} \frac{G_{-}(\lambda, \xi) \sigma(\mu)-G_{-}(\mu, \xi) \sigma(\lambda)}{\operatorname{sh}(\lambda-\mu-\eta)} .
\end{gathered}
$$

This is already a quite remarkable formula. The prefactor nicely combines with the corresponding prefactor in our expression $(55)$ for $F_{+}(\xi)$. The double integral is much reminiscent of the double integral representing the inhomogeneous neighbour correlation function $\left\langle\sigma_{1}^{-} \sigma_{2}^{+}\right\rangle$derived in [4]. In particular, its structure is such that it can be factorized by means of the technique suggested in [4,6].

For this purpose we first note that

$$
\mathrm{d} \bar{m}_{-}^{\alpha}(\lambda)=\frac{\mathrm{d} \lambda}{2 \pi \mathrm{i} \rho_{n}(\lambda \mid \alpha)}-\mathrm{d} m_{-}^{\alpha}(\lambda)
$$


Then

$$
\begin{gathered}
I=\int_{\mathfrak{C}_{n}} \mathrm{~d} m_{-}^{\alpha}(\lambda) \mathrm{e}^{\lambda} \int_{\mathcal{C}_{n}} \mathrm{~d} \bar{m}_{-}^{\alpha}(\mu) \mathrm{e}^{\mu} \frac{G_{-}(\lambda, \xi) \sigma(\mu)-G_{-}(\mu, \xi) \sigma(\lambda)}{\operatorname{sh}(\lambda-\mu-\eta)} \\
=\mathrm{e}^{\xi} \rho_{n}^{-1}(\xi \mid \alpha) \int_{\mathcal{C}_{n}} \mathrm{~d} m_{-}^{\alpha}(\lambda) \frac{\mathrm{e}^{\lambda} \sigma(\lambda)}{\operatorname{sh}(\lambda-\xi-\eta)} \\
-\int_{\mathcal{C}_{n}} \mathrm{~d} m_{-}^{\alpha}(\lambda) \int_{\mathcal{C}_{n}} \mathrm{~d} m_{-}^{\alpha}(\mu) \frac{\mathrm{e}^{\lambda+\mu}}{\operatorname{sh}(\lambda-\mu-\eta)} \operatorname{det}\left(\begin{array}{cc}
G_{-}(\lambda, \xi) & G_{-}(\mu, \xi) \\
\sigma(\lambda) & \sigma(\mu)
\end{array}\right) .
\end{gathered}
$$

Define

$$
r(\lambda, \mu)=\frac{\mathrm{e}^{\lambda+\mu}}{\operatorname{sh}(\lambda-\mu-\eta)}, \quad s(\lambda, \mu)=-\frac{q^{1+\alpha}-q^{-1-\alpha}+K_{1+\alpha}(\lambda-\mu)}{q^{\alpha}-q^{-\alpha}} .
$$

We observe that $r(\lambda, \mu)-\mathrm{e}^{2 \lambda} s(\lambda, \mu)$ is a symmetric function. Hence, we can replace $r(\lambda, \mu)$ by $\mathrm{e}^{2 \lambda} s(\lambda, \mu)$ under the double integral. Then, further using the integral equations B.30 and $(\mathrm{B} .45)$ as well as the asymptotics $\mathrm{B} .33$ and the elementary identity

$$
\begin{aligned}
& \frac{q^{\alpha}-q^{-\alpha}}{\operatorname{sh}(\lambda-\xi-\eta)} \\
& \quad=q^{\alpha+1} \mathrm{e}^{-\lambda+\xi}(\operatorname{cth}(\lambda-\xi-\eta)+1)-q^{-\alpha-1} \mathrm{e}^{\lambda-\xi}(\operatorname{cth}(\lambda-\xi-\eta)-1),
\end{aligned}
$$

we obtain

$$
\begin{aligned}
I= & \frac{G_{-}^{+}(\xi)}{q^{\alpha}-q^{-\alpha}} \int_{\mathcal{C}_{n}} \mathrm{~d} m_{-}^{\alpha}(\lambda) \mathrm{e}^{2 \lambda} \sigma(\lambda) \\
& +\frac{\mathrm{e}^{2 \xi}}{q^{\alpha}-q^{-\alpha}} \int_{\mathfrak{C}_{n}} \mathrm{~d} m_{-}^{\alpha}(\lambda) \sigma(\lambda)\left(-\operatorname{cth}(\lambda-\xi)+q^{\alpha+1} \rho_{n}^{-1}(\xi \mid \alpha) \operatorname{cth}(\lambda-\xi-\eta)\right. \\
& \left.-1+q^{\alpha+1} \rho_{n}^{-1}(\xi \mid \alpha)\right) .
\end{aligned}
$$

Equation B.48) states that $G_{-}^{+}(\xi)=0$ or the integral is zero. For $M=1$ it is easy to see that the integral in $\mathrm{B} .48$ is non-zero. Hence, $G_{-}^{+}(\xi)=0$. We have verified this numerically for more examples and in the following assume it to be generally true. Then $I$ is reduced to the second term in (B.54).

Introducing a function $\bar{G}_{-}$as the solution of the linear integral equation

$$
\begin{aligned}
\bar{G}_{-}(\lambda, \xi) & =-\operatorname{cth}(\lambda-\xi) \\
& +q^{\alpha+1} \rho_{n}^{-1}(\xi \mid \alpha) \operatorname{cth}(\lambda-\xi-\eta)+\int_{\mathfrak{C}_{n}} \mathrm{~d} m_{-}^{\alpha}(\mu) \bar{G}_{-}(\mu, \xi) K_{1+\alpha}(\mu-\lambda)
\end{aligned}
$$

and using the dressed function trick

$$
I=\frac{\mathrm{e}^{2 \xi} \bar{G}_{-}^{+}(\xi)}{\left(q^{\alpha}-q^{-\alpha}\right)\left(q^{\alpha+1}-q^{-\alpha-1}\right)},
$$

where

$$
\bar{G}_{-}^{+}(\xi)=\lim _{\operatorname{Re} \lambda \rightarrow+\infty} \bar{G}_{-}(\lambda, \xi) .
$$

\footnotetext{
${ }^{\dagger}$ Schematically: $f_{1}=g_{1}+\int K f_{1}$ and $f_{2}=g_{2}+\int K^{t} f_{2}$ implies $\int f_{1} g_{2}=\int f_{2} g_{1}$.
} 
This completes our derivation of equation (61) of the main text.

We expect that

$$
\lim _{\alpha \rightarrow 0} \bar{G}_{-}^{+}(\xi)=0
$$

for $I$ to be finite. For the verification note that $\alpha=0$ in $(\overline{B .53})$ implies the identity

$$
K_{1}(\lambda)=\mathrm{e}^{-2 \lambda}\left[K_{-1}(\lambda)-q^{-1}+q\right]+q^{-1}-q
$$

which allows us to replace the kernel $K_{1}$ by $K_{-1}$ in (B.23a). Proceeding with the calculation the function $\bar{G}_{-}^{+}$(for $\alpha=0$ ) along with a modified function $\bar{\sigma}$ will appear instead of $G_{-}^{+}$and $\sigma$. For these functions an identity similar to (B.48) can be derived which implies (B.58).

\section{Appendix C: A generalized Sommerfeld lemma}

In this appendix we provide a proof of Lemma 1 . Let $F$ be an antiderivative of $f$. Using integration by parts we obtain

$$
\begin{aligned}
T \int_{\mathcal{C}_{u}^{-}} \mathrm{d} \lambda & f(\lambda) \ln \left(1+\mathrm{e}^{-\frac{u(\lambda)}{T}}\right) \\
= & T \ln (2)\left(F\left(Q_{+}\right)-F\left(Q_{-}\right)\right)+\int_{Q_{-}}^{Q_{+}} \mathrm{d} \lambda \frac{F(\lambda) u^{\prime}(\lambda)}{1+\mathrm{e}^{\frac{u(\lambda)}{T}}} \\
& =T \int_{\mathcal{C}_{u}^{-}} \mathrm{d} \lambda f(\lambda) \ln \left(1+\mathrm{e}^{\frac{u(\lambda)}{T}}\right)+\int_{Q_{-}}^{Q_{+}} \mathrm{d} \lambda F(\lambda) u^{\prime}(\lambda) \\
& =T \int_{\mathcal{C}_{u}^{-}} \mathrm{d} \lambda f(\lambda) \ln \left(1+\mathrm{e}^{\frac{u(\lambda)}{T}}\right)-\int_{Q_{-}}^{Q_{+}} \mathrm{d} \lambda f(\lambda)(u(\lambda)-2 \pi \mathrm{i} p T) .
\end{aligned}
$$

Setting $\bar{u}(\lambda)=u(\lambda)-2 \pi \mathrm{i} p T$ it follows that

$$
\begin{aligned}
\Delta I=T \int_{\mathcal{C}_{u}} \mathrm{~d} \lambda f(\lambda) \ln \left(1+\mathrm{e}^{-\frac{u(\lambda)}{T}}\right)+\int_{Q_{-}}^{Q_{+}} \mathrm{d} \lambda f(\lambda) \bar{u}(\lambda) \\
\quad=T \int_{\mathcal{C}_{u}} \mathrm{~d} \lambda f(\lambda) \ln \left(1+\mathrm{e}^{-\frac{u(\lambda) \operatorname{sinn}(v(\lambda))}{T}}\right) .
\end{aligned}
$$

Then the real part of the exponent on the right hand side is always negative except at $Q_{ \pm}$. This means that the leading contribution to the integral for $T \rightarrow 0$ comes from the (infinitesimally small) vicinities of these two points. In order to quantify the leading contribution we fix $\delta>0$ small enough. Since $u$ and $f$ are holomorphic we can deform the contour locally in a small vicinity of $Q_{ \pm}$into contours $J_{ \pm}^{\delta}$ such that $w(\lambda)=2 \pi p T$ for $\lambda \in J_{ \pm}^{\delta}$ and $v(\lambda)= \pm \delta$ at the boundaries of $J_{ \pm}^{\delta}$. Note that $v$ is monotonic on $J_{ \pm}^{\delta}$, since it has simple zeros at $Q_{ \pm}$. It follows that

$$
\Delta I=T \int_{J_{-}^{\delta} \cup J_{+}^{\delta}} \mathrm{d} \lambda f(\lambda) \ln \left(1+\mathrm{e}^{-\frac{|v(\lambda)|}{T}}\right)+\mathcal{O}\left(T^{\infty}\right) .
$$

We parameterize $J_{ \pm}^{\delta}$ by $x=v(\lambda) \Leftrightarrow \lambda=v^{-1}(x)$. Then

$$
\begin{array}{r}
T \int_{J_{-}^{\delta}} \mathrm{d} \lambda f(\lambda) \ln \left(1+\mathrm{e}^{-\frac{|v(\lambda)|}{T}}\right)=T \int_{\delta}^{-\delta} \mathrm{d} x \frac{f\left(v^{-1}(x)\right)}{v^{\prime}\left(v^{-1}(x)\right)} \ln \left(1+\mathrm{e}^{-\frac{|x|}{T}}\right) \\
=-\frac{T^{2} \pi^{2}}{6} \frac{f\left(Q_{-}\right)}{u^{\prime}\left(Q_{-}\right)}+\mathcal{O}\left(T^{4}\right) .
\end{array}
$$


Here we have used that $v^{-1}(0)=Q_{-}$and that $\ln \left(1+\mathrm{e}^{-|x|}\right)$ is even. At $Q_{+}$we can perform a similar calculation with the only difference that $v(\lambda)$ is ascending in the direction of the contour, whence the sign will be positive. This completes the proof of the lemma.

\section{Appendix D: Properties of the Fredholm determinants}

In this appendix we obtain alternative forms of the Fredholm determinants or their ratios and discuss the zero-temperature limit of the determinants appearing in the numerator of the determinant part of the amplitudes. For the sake of brevity we restrict ourselves to the longitudinal case.

\section{D.1 On the zero-temperature limit}

In order to calculate the $T \rightarrow 0+$ limit, we note that the $T Q$-equation implies (see [5])

$$
\begin{aligned}
\frac{1}{\rho_{n}(\lambda \mid \alpha)\left(1+\mathfrak{a}_{0}(\lambda)\right)}=-\frac{q^{-\alpha} \phi(\lambda+\eta)}{\rho_{n}(\lambda \mid \alpha)\left(q^{\alpha} \phi(\lambda-\eta)-q^{-\alpha} \phi(\lambda+\eta)\right)} \\
+\frac{\phi(\lambda)}{q^{\alpha} \phi(\lambda-\eta)-q^{-\alpha} \phi(\lambda+\eta)}
\end{aligned}
$$

where $\phi$ is the ratio of $Q$-functions defined in $(\mathrm{A} .2)$. The function on the left hand side is the weight function in the measure $\mathrm{d} m_{-}^{\alpha}$. The function $\phi(\lambda+\eta) / \rho_{n}(\lambda \mid \alpha)$ has no poles inside $\mathcal{C}_{n}$. We shall assume that the contour of the Fredholm determinant with measure $\mathrm{d} m_{-}^{\alpha}$ can be deformed into a contour $\Gamma_{n}^{(-)}$, containing no zero of $q^{\alpha} \phi(\lambda-\eta)-$ $q^{-\alpha} \phi(\lambda+\eta)$ and no pole of the kernel, without changing the value of the determinant. In the Fredholm determinant with this deformed contour we can use (D.1) to replace the measure $\mathrm{d} m_{-}^{\alpha}$ by

$$
\mathrm{d} M_{-}^{\alpha}(\lambda)=\frac{\mathrm{d} \lambda}{2 \pi \mathrm{i}} \frac{\phi(\lambda)}{q^{\alpha} \phi(\lambda-\eta)-q^{-\alpha} \phi(\lambda+\eta)} .
$$

In fact, we expect most of the zeros of $q^{\alpha} \phi(\lambda-\eta)-q^{-\alpha} \phi(\lambda+\eta)$ to be located close to $\pm \eta$, where the essential singularities of the functions $\phi(\lambda \pm \eta)$ appear in the Trotter limit. Thus, $\Gamma_{n}^{(-)}$might be similar to $\mathcal{C}_{n}$.

The weight function in the new measure is easily re-expressed in terms of the auxiliary functions,

$$
\frac{\phi(\lambda)}{q^{\alpha} \phi(\lambda-\eta)-q^{-\alpha} \phi(\lambda+\eta)}=\frac{1+\mathfrak{a}_{n}(\lambda \mid \alpha)}{\rho_{n}(\lambda \mid \alpha)\left(1+\mathfrak{a}_{0}(\lambda)\right)} \frac{1}{1-\mathfrak{a}_{n}(\lambda \mid \alpha) / \mathfrak{a}_{0}(\lambda)} .
$$

We rewrite the factors on the right hand side in a form that is appropriate for performing the zero temperature limit. Using the non-linear integral equation (21) we obtain

$$
\frac{\mathfrak{a}_{n}(\lambda \mid \alpha)}{\mathfrak{a}_{0}(\lambda)}=q^{-2 \alpha} \exp \left\{\int_{\mathfrak{C}_{n}} \mathrm{~d} \mu K(\mu-\lambda) z(\mu)\right\}
$$

while (24) implies that

$$
\frac{1+\mathfrak{a}_{n}(\lambda \mid \alpha)}{\rho_{n}(\lambda \mid \alpha)\left(1+\mathfrak{a}_{0}(\lambda)\right)}=q^{-\alpha} \exp \left\{\int_{\mathfrak{C}_{n}} \mathrm{~d} \mu \mathrm{e}(\mu-\lambda) z(\mu)\right\},
$$


where $\lambda$ is now outside $\mathcal{C}_{n}$.

We now assume that we may shift the contour $\Gamma_{n}^{(-)}$, such that its upper part is slightly above $\mathcal{C}_{0}$, uniformly for all $T$. Clearly the poles of the function $\phi$ do not prevent us from shifting the contour, but we must further assume that $q^{\alpha} \phi(\lambda-\eta)-q^{-\alpha} \phi(\lambda+\eta)$ has no zeros close to $\mathcal{C}_{0}$ for $T \rightarrow 0+$. For the zero temperature limit of the Fredholm determinant with measure $\mathrm{d} M_{-}^{\alpha}$ it is then enough to perform the limit in (D.4) and (D.5) for $\lambda$ away from $C_{0}$. First straightening the contours in D.4 and (D.5) and then using corollary 1 as well as (104) and 98a) we obtain

$$
\begin{aligned}
& \int_{\mathcal{C}_{n}} \mathrm{~d} \mu K(\mu-\lambda) z(\mu)=2 \pi \mathrm{i}\left(\alpha^{\prime}-\ell\right)-2 \pi \mathrm{i}\left(\alpha^{\prime}-\ell\right) Z(\lambda-\mathrm{i} \gamma / 2)+\mathcal{O}(T), \\
& \int_{\mathcal{C}_{n}} \mathrm{~d} \mu \mathrm{e}(\mu-\lambda) z(\mu)=-\left(\alpha^{\prime}-\ell\right) \int_{-Q}^{Q} \mathrm{~d} \mu \mathrm{e}(\mu+\mathrm{i} \gamma / 2-\lambda) Z(\mu)+\mathcal{O}(T)
\end{aligned}
$$

which fixes $\lim _{T \rightarrow 0+} \mathrm{d} M_{-}^{\alpha}(\lambda)$ for $\lambda \in \Gamma_{n}^{(-)}$.

A similar reasoning works for $\mathrm{d} m_{+}^{\alpha}$. The identity

$$
\begin{aligned}
\frac{\rho_{n}(\lambda \mid \alpha)}{1+\mathfrak{a}_{n}(\lambda \mid \alpha)}=-\frac{q^{\alpha} \phi^{-1}(\lambda+\eta) \rho_{n}(\lambda \mid \alpha)}{q^{-\alpha} \phi^{-1}(\lambda-\eta)-q^{\alpha} \phi^{-1}(\lambda+\eta)} & \\
& +\frac{\phi^{-1}(\lambda)}{q^{-\alpha} \phi^{-1}(\lambda-\eta)-q^{\alpha} \phi^{-1}(\lambda+\eta)}
\end{aligned}
$$

inspires the definition of the measure

$$
\begin{aligned}
\mathrm{d} M_{+}^{\alpha}(\lambda)=\frac{\mathrm{d} \lambda}{2 \pi \mathrm{i}} \frac{\phi^{-1}(\lambda)}{q^{-\alpha} \phi^{-1}(\lambda-\eta)-q^{\alpha} \phi^{-1}(\lambda+\eta)} \\
=\frac{\mathrm{d} \lambda}{2 \pi \mathrm{i}} \frac{\rho_{n}(\lambda \mid \alpha)\left(1+\mathfrak{a}_{0}(\lambda)\right)}{1+\mathfrak{a}_{n}(\lambda \mid \alpha)} \frac{1}{1-\mathfrak{a}_{0}(\lambda) / \mathfrak{a}_{n}(\lambda \mid \alpha)}
\end{aligned}
$$

which replaces $\mathrm{d} m_{+}^{\alpha}(\lambda)$ on an appropriate contour $\Gamma_{n}^{(+)}$. We assume it can be kept away from $\mathcal{C}_{0}$. Then eqs. D.6 determine the zero temperature limit of $\mathrm{d} M_{+}^{\alpha}$ on $\Gamma_{n}^{(+)}$.

Our claims can be summarized as follows. Define

$$
\mathrm{d} \widehat{M}_{ \pm}^{\alpha}(\lambda)=\frac{\mathrm{d} \lambda}{2 \pi \mathrm{i}} \frac{\mathrm{e}^{ \pm i \pi \alpha^{\prime} \pm\left(\alpha^{\prime}-\ell\right) \int_{-Q}^{Q} \mathrm{~d} \mu \mathrm{e}(\mu-\lambda) Z(\mu)}}{1-\mathrm{e}^{ \pm 2 \pi \mathrm{i}\left(\alpha^{\prime}-\ell\right) Z(\lambda)}}
$$

Then

$$
\lim _{T \rightarrow 0+} \mathrm{d} M_{ \pm}^{\alpha}(\lambda)=\mathrm{d} \widehat{M}_{ \pm}^{\alpha}(\lambda-\mathrm{i} \gamma / 2)
$$

In order to obtain the formulae in the main text it remains to shrink the contours $\Gamma_{n}^{( \pm)}$to a contour $\Gamma[-Q, Q]+\mathrm{i} \gamma / 2$, encircling $[-Q, Q]+\mathrm{i} \gamma / 2$ in counterclockwise manner and to shift this down to the real axis.

Note that $Z(\lambda)$ is real on the real axis. Therefore, in accordance with our assumptions, the denominator in D.9 is nonzero on the real axis, as long as $\alpha^{\prime}$ has a non-vanishing imaginary part. 


\section{D.2 Relation to previous determinant formulae}

Using some of the results of the previous subsection we can rewrite the determinants in the numerator and establish a relation with the determinants obtained in [25, 37, 38]. For this purpose we first note that by construction

$$
\operatorname{det}_{\mathrm{d} m_{ \pm}^{\alpha}, e_{n}}\left\{1-\widehat{\mathcal{K}}_{\mp \alpha}\right\}=\operatorname{det}_{\mathrm{d} M_{ \pm}^{\alpha}, \Gamma_{n}^{( \pm)}}\left\{1-\widehat{\mathcal{K}}_{\mp \alpha}\right\} .
$$

Using eqs. (D.4) and (D.5) and inserting the definition (122) of the periodic Cauchy transform, we rewrite $\mathrm{d} M_{ \pm}^{\alpha}$ as

$$
\mathrm{d} M_{ \pm}^{\alpha}(\lambda)=\mp \frac{\mathrm{d} \lambda}{2 \pi \mathrm{i}} \frac{q^{-\alpha} \mathrm{e}^{\mp L_{\mathrm{C}_{n}}[z](\lambda)}}{\mathrm{e}_{\mathrm{e}_{n}}[z](\lambda \pm \eta)}-q^{-2 \alpha} \mathrm{e}^{\mp L_{\mathrm{C}_{n}}[z](\lambda \mp \eta)} .
$$

Due to (41) and (47) we may replace $\mathcal{K}_{-\alpha}(\lambda)$ by $K_{-\alpha}(\lambda)-q^{\alpha}+q^{-\alpha}$ and $\mathcal{K}_{\alpha}(\lambda)$ by $K_{\alpha}(-\lambda)-q^{-\alpha}+q^{\alpha}$ in the determinants in (D.11).

We define $\beta=q^{-2 \alpha}$,

$$
\widetilde{K}_{\beta}(\lambda)=\operatorname{cth}(\lambda-\eta)-\beta \operatorname{cth}(\lambda+\eta)
$$

and two new kernels

$$
\begin{aligned}
& U_{\theta}^{(-)}(\lambda, \mu)=-\frac{1}{2 \pi \mathrm{i}} \frac{\mathrm{e}^{L_{e_{n}}[z](\lambda)}\left(\widetilde{K}_{\beta}(\lambda-\mu)-\widetilde{K}_{\beta}(\theta-\mu)\right)}{\mathrm{e}^{L_{e_{n}}[z](\lambda-\eta)}-\beta \mathrm{e}^{L_{\mathfrak{e}_{n}}[z](\lambda+\eta)}}, \\
& U_{\theta}^{(+)}(\lambda, \mu)=\frac{1}{2 \pi \mathrm{i}} \frac{\mathrm{e}^{-L_{e_{n}}[z](\mu)}\left(\widetilde{K}_{\beta}(\lambda-\mu)-\widetilde{K}_{\beta}(\lambda-\theta)\right)}{\mathrm{e}^{-L_{e_{n}}[z](\mu+\eta)}-\beta \mathrm{e}^{-L_{e_{n}}[z](\mu-\eta)}} .
\end{aligned}
$$

Then clearly

$$
\operatorname{det}_{\mathrm{d} m_{ \pm}^{\alpha}, \mathcal{C}_{n}}\left\{1-\widehat{\mathcal{K}}_{\mp \alpha}\right\}=\lim _{\theta \rightarrow-\infty} \operatorname{det}_{\mathrm{d} \lambda, \Gamma_{n}^{( \pm)}}\left\{1+\widehat{U}_{\theta}^{( \pm)}\right\} .
$$

In order to compare with [37,38] we note that

$$
\lim _{\theta \rightarrow-\infty} \mathrm{e}^{L_{\mathcal{C}_{n}}[z](\theta \pm \eta)}=\mathrm{e}^{\int_{\mathcal{E}_{n}} \mathrm{~d} \lambda z(\lambda)}=\lim _{\operatorname{Re} \lambda \rightarrow-\infty} \phi(\lambda)=b^{-1},
$$

whence

$$
\lim _{\theta \rightarrow-\infty} \frac{b^{ \pm 1}(1-\beta)}{\mathrm{e}^{\mp L_{e_{n}}[z](\theta \pm \eta)}-\beta \mathrm{e}^{\mp L_{e_{n}}[z](\theta \mp \eta)}}=1,
$$

and therefore

$$
\operatorname{det}_{\mathrm{d} m_{ \pm}^{\alpha}, \mathrm{e}_{n}}\left\{1-\widehat{\mathcal{K}}_{\mp \alpha}\right\}=\lim _{\theta \rightarrow-\infty}\left[\frac{b^{ \pm 1}(1-\beta)}{\mathrm{e}^{\mp L \mathcal{C}_{n}[z](\theta \pm \eta)}-\beta \mathrm{e}^{\mp L e_{n}[z](\theta \mp \eta)}} \underset{\mathrm{d} \lambda, \Gamma_{n}^{( \pm)}}{\operatorname{det}}\left\{1+\widehat{U}_{\theta}^{( \pm)}\right\}\right] .
$$

Using exactly the same reasoning as in appendix A.3 of [22] one can show that the expression in square brackets on the right hand side is independent of $\theta$. Hence,

$$
\operatorname{det}_{\mathrm{d} m_{ \pm}^{\alpha}, \mathcal{C}_{n}}\left\{1-\widehat{\mathcal{K}}_{\mp \alpha}\right\}=\frac{b^{ \pm 1}(1-\beta)}{\mathrm{e}^{\mp L_{C_{n}}[z](\theta \pm \eta)}-\beta \mathrm{e}^{\mp L_{e_{n}}[z](\theta \mp \eta)}} \underset{\mathrm{d} \lambda, \Gamma_{n}^{( \pm)}}{\operatorname{det}}\left\{1+\widehat{U}_{\theta}^{( \pm)}\right\} .
$$

Introducing two independent parameters $\theta_{1}$ and $\theta_{2}$ for the two cases, the determinants take the same form as in [37,38] (see e.g. eqs. (2.14)-(2.18) in [37]). Taking the limit $T \rightarrow 0+$ and setting $\theta_{1}=-Q, \theta_{2}=Q$ we reproduce the expressions obtained in [22 [25]. 


\section{D.3 Dependence on the twist parameter}

Equation (D.19) is important, because it allows us to understand the $\alpha$ dependence of the determinants. Recalling that $\beta=q^{-2 \alpha}$ we infer that $\operatorname{det}_{\mathrm{d} m}^{\alpha}, \mathcal{C}_{n}\left\{1-\widehat{\mathcal{K}}_{\mp \alpha}\right\}=\mathcal{O}(\alpha)$ unless $n=0$.

This statement remains true for $T \rightarrow 0+$ unless $\ell=0$. The case $\ell=0$ for $T \rightarrow 0+$ needs a more careful treatment (compare [22,38]). Consider (133) and (134) for $\ell=0$. Recall that the contour $\Gamma[-Q, Q]$ encircles the interval $[-Q, Q]$ counterclockwise. Thus, we may replace the contour by $[-Q, Q]$ if we replace at the same time the weight by

$$
\mathrm{d} \breve{M}_{ \pm}^{\alpha}(\lambda)=\frac{\mathrm{d} \lambda \mathrm{e}^{ \pm \mathrm{i} \pi \alpha^{\prime}}}{2 \pi \mathrm{i}\left(1-\mathrm{e}^{ \pm 2 \pi \mathrm{i} \alpha^{\prime} Z(\lambda)}\right)}\left(\mathrm{e}^{ \pm \alpha^{\prime} \int_{-Q}^{Q} \mathrm{~d} \mu \mathrm{e}\left(\mu-\lambda_{-}\right) Z(\mu)}-\mathrm{e}^{ \pm \alpha^{\prime} \int_{-Q}^{Q} \mathrm{~d} \mu \mathrm{e}\left(\mu-\lambda_{+}\right) Z(\mu)}\right) .
$$

Here $\lambda_{+}$denotes the limit from above and $\lambda_{-}$the limit from below. This replacement is justified since the right hand side of (D.20) can be recast as

$$
\mathrm{d} \breve{M}_{ \pm}^{\alpha}(\lambda)=\frac{\mathrm{d} \lambda}{2 \pi \mathrm{i}}\left(\frac{\operatorname{sh}\left(\lambda_{-}-Q\right) \operatorname{sh}(\lambda+Q-\eta)}{\operatorname{sh}\left(\lambda_{-}+Q\right) \operatorname{sh}(\lambda-Q-\eta)}\right)^{ \pm \alpha^{\prime} z} \mathrm{e}^{ \pm \mathrm{i} \pi \alpha^{\prime} \pm \alpha^{\prime} \int_{-Q}^{Q} \mathrm{~d} \mu \mathrm{e}\left(\mu-\lambda_{-}\right)(Z(\mu)-z)}
$$

and since for small $\alpha^{\prime}$ the singularity of the latter expression can be integrated over. We further conclude that $\lim _{\alpha \rightarrow 0} \mathrm{~d} \breve{M}_{ \pm}^{\alpha}(\lambda)=\mathrm{d} \lambda / 2 \pi \mathrm{i}$.

For the ratio of determinants $\mathcal{D}(\ell)$ defined in 135 ) eq. (D.21) implies that

$$
\mathcal{D}(0)=1+\mathcal{O}\left(\alpha^{2}\right)
$$

which is needed for calculating the longitudinal correlation functions from the generating function.

\section{D.4 A quotient formula}

This subsection is of auxiliary character. We would like to point out that the ratios of Fredholm determinants in the main text can be combined into single Fredholm determinants. This may turn out to be useful e.g. in the low-temperature analysis of the model for vanishing magnetic field.

Consider, for instance, the ratio

$$
\mathcal{D}_{-}=\frac{\operatorname{det}_{M}\left\{\delta_{k}^{j}-\frac{\rho_{n}^{-1}\left(\lambda_{j} \mid \alpha\right)}{\mathfrak{a}_{0}^{\prime}\left(\lambda_{j}\right)} \mathcal{K}_{\alpha}\left(\lambda_{j}-\lambda_{k}\right)\right\}}{\operatorname{det}_{M}\left\{\delta_{k}^{j}-\frac{1}{\mathfrak{a}_{0}^{\prime}\left(\lambda_{j}\right)} \mathcal{K}\left(\lambda_{j}-\lambda_{k}\right)\right\}}
$$

occurring in eq. (38). We would like to write it as a single Fredholm-type determinant of the form

$$
\mathcal{D}_{-}=\operatorname{det}_{M}^{-1}\left(\mathbf{e}_{1}+\mathbf{w}_{1}, \ldots, \mathbf{e}_{M}+\mathbf{w}_{M}\right),
$$

where the $\mathbf{e}_{k}$ denote the canonical unit vectors, $\mathbf{e}_{k}^{j}=\delta_{k}^{j}$ and where the $\mathbf{w}_{k}$ are to be determined. Comparing (D.23) and (D.24) we see that they must satisfy the equations

$$
\begin{aligned}
\mathbf{w}_{k}^{j} \mathfrak{a}_{0}^{\prime}\left(\lambda_{j}\right)=\mathcal{K}_{\alpha}\left(\lambda_{j}-\lambda_{k}\right) \rho_{n}^{-1}\left(\lambda_{k} \mid \alpha\right)-\mathcal{K}\left(\lambda_{j}-\lambda_{k}\right) & \\
& +\sum_{\ell=1}^{M} \mathcal{K}_{\alpha}\left(\lambda_{j}-\lambda_{\ell}\right) \rho_{n}^{-1}\left(\lambda_{\ell} \mid \alpha\right) \mathbf{w}_{\ell}^{j} .
\end{aligned}
$$


These can be turned into an integral equation. Defining

$$
F(\lambda, v)=\mathcal{K}_{\alpha}(\lambda-v) \rho_{n}^{-1}(v \mid \alpha)-\mathcal{K}(\lambda-v)+\int_{\mathcal{C}_{n}} \mathrm{~d} m_{-}^{\alpha}(\mu) \mathcal{K}_{\alpha}(\lambda-\mu) F(\mu, v)
$$

we conclude that $\mathbf{w}_{k}^{j}=F\left(\lambda_{j}, \lambda_{k}\right) / \mathfrak{a}_{0}^{\prime}\left(\lambda_{j}\right)$. Thus,

$$
\mathcal{D}_{-}=\operatorname{det}_{M}^{-1}\left\{\delta_{k}^{j}+\frac{F\left(\lambda_{j}, \lambda_{k}\right)}{\mathfrak{a}_{0}^{\prime}\left(\lambda_{j}\right)}\right\} \rightarrow \operatorname{det}_{\mathrm{d} m, \mathrm{C}_{n}}^{-1}\{1+\widehat{F}\} .
$$

In a similar way we obtain the alternative representation

$$
\mathcal{D}_{-}=\operatorname{det}_{M}\left\{\delta_{k}^{j}+\frac{\bar{F}\left(\lambda_{j}, \lambda_{k}\right)}{\mathfrak{a}_{0}^{\prime}\left(\lambda_{j}\right)}\right\} \longrightarrow \operatorname{det}_{\mathrm{d} m, \mathrm{C}_{n}}\{1+\widehat{\bar{F}}\},
$$

where

$$
\bar{F}(\lambda, v)=\mathcal{K}(\lambda-v)-\mathcal{K}_{\alpha}(\lambda-v) \rho_{n}^{-1}(v \mid \alpha)+\int_{\mathcal{C}_{n}} \mathrm{~d} m(\mu) \mathcal{K}(\lambda-\mu) \bar{F}(\mu, v) .
$$

Similar expressions can also be obtained for the other ratio of determinants in 38.

\section{References}

[1] N. M. Bogolyubov, A. G. Izergin, and V. E. Korepin, Critical exponents for integrable models, Nucl. Phys. B 275 (1986), 687.

[2] H. Boos, Fermionic basis in conformal field theory and thermodynamic Bethe ansatz for excited states, SIGMA 7 (2011), 007 (36 pp).

[3] H. Boos, J. Damerau, F. Göhmann, A. Klümper, J. Suzuki, and A. Weiße, Shortdistance thermal correlations in the XXZ chain, J. Stat. Mech.: Theor. Exp. (2008), $\mathrm{P} 08010$.

[4] H. Boos and F. Göhmann, On the physical part of the factorized correlation functions of the XXZ chain, J. Phys. A 42 (2009), 315001.

[5] _ Properties of linear integral equations related to the six-vertex model with disorder parameter, New Trends in Quantum Integrable Systems (Boris Feigin, Michio Jimbo, and Masato Okado, eds.), World Scientific, Singapore, 2010, Proceedings of the conference on Infinite Analysis 09, pp 1-10.

[6] H. Boos, F. Göhmann, A. Klümper, and J. Suzuki, Factorization of multiple integrals representing the density matrix of a finite segment of the Heisenberg spin chain, J. Stat. Mech.: Theor. Exp. (2006), P04001.

[7] _ Factorization of the finite temperature correlation functions of the XXZ chain in a magnetic field, J. Phys. A 40 (2007), 10699.

[8] H. Boos, M. Jimbo, T. Miwa, and F. Smirnov, Hidden Grassmann structure in the XXZ model IV: CFT limit, Comm. Math. Phys. 299 (2010), 825. 
[9] H. Boos, M. Jimbo, T. Miwa, F. Smirnov, and Y. Takeyama, Hidden Grassmann structure in the XXZ model II: creation operators, Comm. Math. Phys. 286 (2009), 875.

[10] J. L. Cardy, Conformal invariance and universality in finite-size scaling, J. Phys. A 17 (1984), L385.

[11] Operator content of two-dimensional conformally invariant theories, Nucl. Phys. B 270 (1986), 186.

[12] J. Damerau, F. Göhmann, N. P. Hasenclever, and A. Klümper, Density matrices for finite segments of Heisenberg chains of arbitrary length, J. Phys. A 40 (2007), 4439.

[13] K. Fabricius, A. Klümper, and B. M. McCoy, Temperature-dependent spatial oscillations in the correlations of the XXZ spin chain, Phys. Rev. Lett. 82 (1999), 5365.

[14] F. Göhmann, A. Klümper, and A. Seel, Integral representations for correlation functions of the XXZ chain at finite temperature, J. Phys. A 37 (2004), 7625.

[15] _ Integral representation of the density matrix of the XXZ chain at finite temperature, J. Phys. A 38 (2005), 1833.

[16] A. G. Izergin, N. Kitanine, J. M. Maillet, and V. Terras, Spontaneous magnetization of the XXZ Heisenberg spin- $\frac{1}{2}$ chain, Nucl. Phys. B 554 (1999), 679.

[17] A. G. Izergin and V. E. Korepin, The quantum inverse scattering method approach to correlation functions, Comm. Math. Phys. 94 (1984), 67.

[18] M. Jimbo and T. Miwa, Algebraic analysis of solvable lattice models, American Mathematical Society, 1995.

[19] M. Jimbo, T. Miwa, and F. Smirnov, Hidden Grassmann structure in the XXZ model III: introducing Matsubara direction, J. Phys. A 42 (2009), 304018.

[20] M. Karowski and P. Weisz, Exact form factors in 1+1-dimensional field theoretic models with soliton behaviour, Nucl. Phys. B 139 (1978), 455.

[21] S. Kerov, G. Olshanski, and A. Vershik, Harmonic analysis on the infinite symmetric group. A deformation of the regular representation, Compt. Rend. Acad. Sci. Paris, Ser. I 316 (1993), 773.

[22] N. Kitanine, K. K. Kozlowski, J. M. Maillet, N. A. Slavnov, and V. Terras, Algebraic Bethe ansatz approach to the asymptotic behavior of correlation functions, J. Stat. Mech.: Theor. Exp. 0904 (2009), P003.

[23] _ On the thermodynamic limit of form factors in the massless XXZ Heisenberg chain, J. Math. Phys. 50 (2009), 095209.

[24]__ A form factor approach to the asymptotic behavior of correlation functions in critical models, J. Stat. Mech.: Theor. Exp. (2011), P12010. 
[25] XXZ Heisenberg chain, J. Stat. Mech.: Theor. Exp. 1105 (2011), P028.

[26] - Form factor approach to dynamical correlation functions in critical models, J. Stat. Mech.: Theor. Exp. (2012), P09001.

[27] N. Kitanine, J. M. Maillet, N. A. Slavnov, and V. Terras, Master equation for spin-spin correlation functions of the XXZ chain, Nucl. Phys. B 712 (2005), 600.

[28] N. Kitanine, J. M. Maillet, and V. Terras, Form factors of the XXZ Heisenberg spin- $\frac{1}{2}$ finite chain, Nucl. Phys. B 554 (1999), 647.

[29] A. Klümper, Free energy and correlation length of quantum chains related to restricted solid-on-solid lattice models, Ann. Physik 1 (1992), 540.

[30] __ Thermodynamics of the anisotropic spin-1/2 Heisenberg chain and related quantum chains, Z. Phys. B 91 (1993), 507.

[31] _ The spin-1/2 Heisenberg chain: thermodynamics, quantum criticality and spin-Peierls exponents, Eur. Phys. J. B 5 (1998), 677.

[32] A. Klümper, M. T. Batchelor, and P. A. Pearce, Central charges of the 6- and 19-vertex models with twisted boundary conditions, J. Phys. A 24 (1991), 3111.

[33] A. Klümper, J. R. Martinez, C. Scheeren, and M. Shiroishi, The spin-1/2 XXZ chain at finite magnetic field: Crossover phenomena driven by temperature, J. Stat. Phys. 102 (2001), 937.

[34] A. Klümper and C. Scheeren, The thermodynamics of the spin-1/2 XXX chain: free energy and low-temperature singularities of correlation lengths, Classical and Quantum Nonlinear Integrable Systems (A. Kundu, ed.), Series in Mathematical and Computational Physics, IOP publishing, Bristol, 2003, pp. 234-255.

[35] V. E. Korepin, Calculation of norms of Bethe wave functions, Comm. Math. Phys. 86 (1982), 391.

[36] V. E. Korepin, N. M. Bogoliubov, and A. G. Izergin, Quantum inverse scattering method and correlation functions, Cambridge University Press, 1993.

[37] K. K. Kozlowski, J. M. Maillet, and N. A. Slavnov, Correlation functions for one-dimensional bosons at low temperature, J. Stat. Mech.: Theor. Exp. 1103 (2011), P019.

[38] Long-distance behavior of temperature correlation functions in the onedimensional Bose gas, J. Stat. Mech.: Theor. Exp. 1103 (2011), P018.

[39] A. Luther and I. Peschel, Calculation of critical exponents in two dimensions from quantum field theory in one dimension, Phys. Rev. B 12 (1975), 3908.

[40] G. Olshanski, Point processes and the infinite symmetric group. Part I: The general formalism and the density function, In: The orbit method in geometry and physics: in honor of A. A. Kirillov (C. Duval, L. Guieu, and V. Ovsienko, eds.), Birkhäuser Verlag, Basel, 2003, Progress in Math. 213. 
[41] M. Reed and B. Simon, Methods of modern mathematical physics IV: Analysis of operators, ch. 13, Academic Press, New York, 1978.

[42] __ Methods of modern mathematical physics I: Functional analysis, second, enlarged ed., ch. 6, Academic Press, New York, 1980.

[43] K. Sakai, M. Shiroishi, J. Suzuki, and Y. Umeno, Commuting quantum transfer matrix approach to intrinsic Fermion system: Correlation length of a spinless Fermion model, Phys. Rev. B 60 (1999), 5186.

[44] J. Sato, B. Aufgebauer, H. Boos, F. Göhmann, A. Klümper, M. Takahashi, and C. Trippe, Computation of static Heisenberg-chain correlators: Control over length and temperature dependence, Phys. Rev. Lett. 106 (2011), 257201.

[45] A. Seel, Die XXZ-Kette: Statische Korrelationen bei endlichen Temperaturen, Ph.D. thesis, Bergische Universität Wuppertal, 2005.

[46] N. A. Slavnov, Calculation of scalar products of the wave functions and form factors in the framework of the algebraic Bethe ansatz, Teor. Mat. Fiz. 79 (1989), 232.

[47] F. A. Smirnov, A general formula for soliton form factors in the quantum sineGordon model, J. Phys. A 19 (1986), L575-L578.

[48] __ Dynamical symmetries of massive integrable models. 1. Form factor bootstrap equations as a special case of deformed Knizhnik-Zamolodchikov equations, Int. J. Mod. Phys. A 7 (1992), S813.

[49] _ Form factors in completely integrable models of quantum field theory, World Scientific, Singapore, 1992.

[50] A. Sommerfeld, Zur Elektronentheorie der Metalle auf Grund der Fermischen Statistik, Z. Phys. 47 (1928), 1.

[51] M. Suzuki, Transfer-matrix method and Monte Carlo simulation in quantum spin systems, Phys. Rev. B 31 (1985), 2957.

[52] M. Suzuki and M. Inoue, The ST-transformation approach to analytic solutions of quantum systems. I. General formulations and basic limit theorems, Prog. Theor. Phys. 78 (1987), 787.

[53] M. Takahashi, Correlation length and free energy of the $S=\frac{1}{2} X Y Z$ chain, Phys. Rev. B 43 (1991), 5788.

[54] C. Trippe, F. Göhmann, and A. Klümper, Short-distance thermal correlations in the massive XXZ chain, Eur. Phys. J. B 73 (2010), 253.

[55] Z. Tsuboi and M. Shiroishi, High temperature expansion of the emptiness formation probability for the isotropic Heisenberg chain, J. Phys. A 38 (2005), L363.

[56] C. N. Yang and C. P. Yang, Ground-state energy of a Heisenberg-Ising lattice, Phys. Rev. 147 (1966), 303. 